\title{
Extreme-ultraviolet light generation in plasmonic nanostructures
}

Plasmonic enhancement of high harmonic generation revisited

\author{
Dissertation \\ zur Erlangung des mathematisch-naturwissenschaftlichen Doktorgrades \\ "Doctor rerum naturalium" \\ der Georg-August-Universität Göttingen \\ im Promotionsprogramm ProPhys \\ der Georg-August University School of Science (GAUSS)
}

vorgelegt von

Herrn Dipl.-Phys. Murat Sivis

aus Kassel

Göttingen, 2013 
$\underline{\text { Betreuungsausschuss }}$

Prof. Dr. Claus Ropers, 4. Physikalisches Institut

Prof. Dr. Markus Münzenberg, 1. Physikalisches Institut

$\underline{\text { Mitglieder der Prüfungskommission }}$

Referent: $\quad$ Prof. Dr. Claus Ropers, 4. Physikalisches Institut

Korreferent: $\quad$ Prof. Dr. Tim Salditt, Institut für Röntgenphysik

Drittgutachter: $\quad$ Prof. Dr. Bernd Abel, Universität Leipzig

$\underline{\text { Weitere Mitglieder der Prüfungskommission }}$

Prof. Dr. Markus Münzenberg, 1. Physikalisches Institut

Prof. Dr. Hans Hofsäss, 2. Physikalisches Institut

Prof. Dr. Jörg Enderlein, 3. Physikalisches Institut

Prof. Dr. Ansgar Reiners, Institut für Astrophysik

Tag der mündlichen Prüfung: 13. November 2013 
There is nothing more deceptive than an obvious fact.

Sir Arthur Conan Doyle, 1891 


\section{Abstract}

The present (cumulative) thesis examines fundamentals of nanostructure-enhanced extreme-ultraviolet light generation in noble gases using two different nanostructure geometries for local field-enhancement. Specifically, resonant antennas and tapered hollow waveguide nanostructures are utilized to enhance low-energy femtosecond laser pulses, which in turn induce light emission from excited xenon, argon and neon atoms and ions. Spectral analysis of this radiation reveals that coherent high-order harmonic generation is not feasible under the examined conditions, contrary to former expectations and reports. Instead, the spectral characteristics unequivocally identify that incoherent fluorescence from multiphoton excited and strong-field ionized gas atoms is the predominant process in such schemes. Furthermore, novel nanostructure-enhanced effects are reported such as surface-enhanced fifth-order harmonic generation (from bow-tie nanoantennas) and the formation of a bistable nanoplasma (in a hollow waveguide). These effects offer intriguing links between nonlinear nano-optics, plasma dynamics and extreme-ultraviolet radiation. 


\section{Contents}

$\begin{array}{ll}\text { List of Figures } & \text { ix }\end{array}$

1 Introduction 1

1.1 Extreme-ultraviolet light generation in atomic gases . . . . . . . . . . 7

1.2 Plasmonic nanostructures for field enhancement . . . . . . . . . . . . . . 13

1.3 Plasmon-enhanced strong-field gas excitation . . . . . . . . . . . . . . 19

1.4 Experimental methods . . . . . . . . . . . . . . 21

2 Nanostructure-enhanced atomic line emission 25

3 Extreme-ultraviolet light generation in plasmonic nanostructures $\quad 29$

4 Generation and Bistability of a Waveguide Nanoplasma Observed by Enhanced Extreme-Ultraviolet Fluorescence $\quad 41$

5 General discussion 51

5.1 Summary . . . . . . . . . . . . . . . . . . 51

5.2 Plasmonic enhancement of $\mathrm{HHG}$ revisited . . . . . . . . . . . . . . 52

5.3 Concept for efficient plasmon-enhanced HHG . . . . . . . . . . . . . . 56

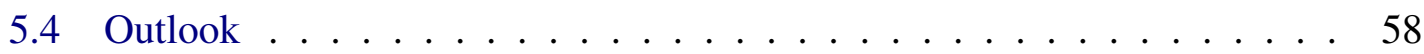

5.4 .1 Gauging of plasmonic near-fields . . . . . . . . . . 58

5.4.2 Surface-enhanced low-order harmonic generation . . . . . . . . 60

5.4.3 Optical bistability of the waveguide nanoplasma . . . . . . . 60

5.4.4 Future applications for EUV fluorescence . . . . . . . . . . 65

$\begin{array}{ll}\text { A Kim et al. reply } & 67\end{array}$

$\begin{array}{ll}\text { Bibliography } & 69\end{array}$ 
Contents

Author contribution

83

Danksagung

85

viii 


\section{List of Figures}

1.1 Experimental setup and nanostructures for plasmon-enhanced HHG . . . 2

1.2 Extreme-ultraviolet emission spectrum . . . . . . . . . . . 3

1.3 Schematic illustration (a) and spectral data (b) for plasmon-enhanced HHG experiments using a hollow waveguide for field enhancement . . . . . . . 4

1.4 Schematic illustration of the fluorescence excitation and radiation mechanisms ........................... 8

1.5 EUV fluorescence spectra from nitrogen and argon gas . . . . . . . . . 9

1.6 Three-step explanation of the simple man's model . . . . . . . . . . . . . 10

1.7 High-order harmonic spectrum . . . . . . . . . . . . . . . . . . . . . . . 12

1.8 Quadratic scaling of the harmonic yield with target density . . . . . . . . 13

1.9 Illustrations of light induced excitation of a plasmonic metal . . . . . . . 14

1.10 Absolute value of the polarizability $|\alpha|$ for a small gold sphere and a gold ellipsoid with high aspect ratio . . . . . . . . . . . . . . 16

1.11 Scheme of a bow-tie antenna and FDTD simulations . . . . . . . . . 17

1.12 Waveguide excitation . . . . . . . . . . . . . . . 17

1.13 FDTD simulations for the intensity enhancement in a tapered hollow wave-

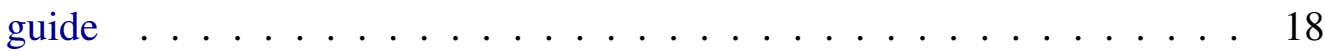

1.14 Schematic illustrations of the two concept for nanostructure-enhanced strong-field gas excitation . . . . . . . . . . . . . . . . . 20

1.15 Experimental setup and excitation schemes for EUV light generation with and without nanostructure-enhancement . . . . . . . . . . . . 21

1.16 Home-built vacuum setup for EUV light generation and spectrally-resolved detection . . . . . . . . . . . . . . . . . . . 23

1.17 Optimization of the gold film roughness for the fabrication of nanoantennas with high structural quality. . . . . . . . . . . . . . . . . . 24

2.1 Experimental set-up and results . . . . . . . . . . . . . . . 26 
3.1 Diagrams of the set-up and generation schemes . . . . . . . . . 31

3.2 Nanostructure-enhanced emission spectra . . . . . . . . . . . . 32

3.3 Durability measurements . . . . . . . . . . . . . . . 34

3.4 Intensity-dependent reference measurements . . . . . . . . . . . . . . 35

4.1 Schematics of the experimental setup/SEM images of the waveguide nanostructures/Third harmonic signal . . . . . . . . . . . . . . . . . . 43

4.2 Nanostructure-enhanced EUV spectra . . . . . . . . . . . . . . . . 45

4.3 Polarization dependence and EUV emission map . . . . . . . . . . . . 46

4.4 Intensity-dependent hysteresis and bistability of the EUV signal . . . . . 49

5.1 Nanostructure-enhanced EUV spectrum by Park et al. . . . . . . . . . . 55

5.2 Concept for efficient plasmon-enhanced HHG by using SPP waves in a channel waveguide .................... 56

5.3 Channel-plasmon-polaritons ................ 57

5.4 Bistability in a inductively-coupled gas discharge plasma . . . . . . . 61

5.5 Ionization paths . . . . . . . . . . . . . . . . . . . 62

5.6 Illustration of the appearance of bistability based on stepwise excitation processes. ........................ 63

5.7 Intensity hysteresis from a fresh and moderately modified waveguide . . . 64

5.8 Possible applications for the waveguide-enhanced EUV emission . . . . . 65 


\section{Chapter 1}

\section{Introduction}

Plasmonic nanostructures enable the spatial confinement of electromagnetic radiation on sub-wavelength scales, leading to orders of magnitude intensity enhancements of incident laser fields. This allows for the study of numerous linear and nonlinear optical processes such as surface-enhanced Raman scattering [1, 2], second [3, 4] and third [5, 6] harmonic generation, and multiphoton photoemission $[7,8]$ using low-energy laser pulses with high $(\mathrm{MHz})$ repetition rates. The rapidly increasing number of reports about the enhancement of optical phenomena in nanoantennas, -waveguides, -tips, -spheres, -particles, and rough surfaces impressively illustrate the growing importance of nano-optics in the natural sciences. Recent review articles summarize progress in the field and highlight the great potential of plasmonic nanostructures to serve as ultimate tools for light control, enhancement and detection $[9,10,11]$.

Most of the reported nanostructure-enhanced implementations rely on light-matter interactions at moderate local intensities, resulting in the excitation of low-order nonlinearities due to electron perturbations in atoms, molecules or solids and surfaces. Recently, also higher-order nonlinear optical phenomena have become accessible in such experiments. Specifically, the so called strong-field regime was reached in nanostructures using local intensities sufficient to trigger field-driven ionization and electron accelerationeffects that have been shown in the context of the strong-field photoelectric effect at metal nanotips, -antennas and -particles [12, 13, 14, 15, 16, 17]. Furthermore, the spatial confinement and inhomogeneity of the enhanced near-fields often lead to alternative, nanostructure-specific scalings of the observed processes, which are at variance from those exhibited by their classical—non-field-enhanced—counterparts.

In this context, the integration of one of the most prominent strong-field effects-high- 
order harmonic generation (HHG) - into a nanostructure-based scenario has created a sensation in the scientific community for the wealth of new physics and potential applications it could enable. High-order harmonic up-conversion of intense laser radiation in gaseous media was first discovered in the late 1980s [18, 19, 20] and allows for new experimental strategies in nanoscale photonic-imaging [21] or ultrafast spectroscopy [22, 23] by providing fully coherent attosecond light pulses at extreme-ultraviolet (EUV) wavelengths. State-of-the-art HHG concepts typically utilize moderately focused, ultrashort laser pulses from $\mathrm{kHz}$ amplifier systems to reach intensities in excess of $10^{13} \mathrm{~W} / \mathrm{cm}^{2}$ (for infrared light frequencies), which are necessary to drive the HHG process in an atomic gas. Using the plasmonic field enhancement of ultrashort, low-energy laser pulses in resonant nanoantennas as described by Kim et al. [24] could greatly simplify the generation of high harmonic radiation. In addition, the much higher generation rates promised in nanostructure-enhanced HHG would have great advantages for ultrafast spectroscopy.

Figure 1.1(a) schematically illustrates the basic experimental approach used by Kim et al. in 2008 in their attempt to realize nanostructure-enhanced HHG. Ultrashort pulses from a Ti:sapphire laser oscillator are focused on an array of gold bow-tie nanoantennas (see scanning electron micrograph in Fig. 1.1(b)), and the intensity is enhanced in the gap

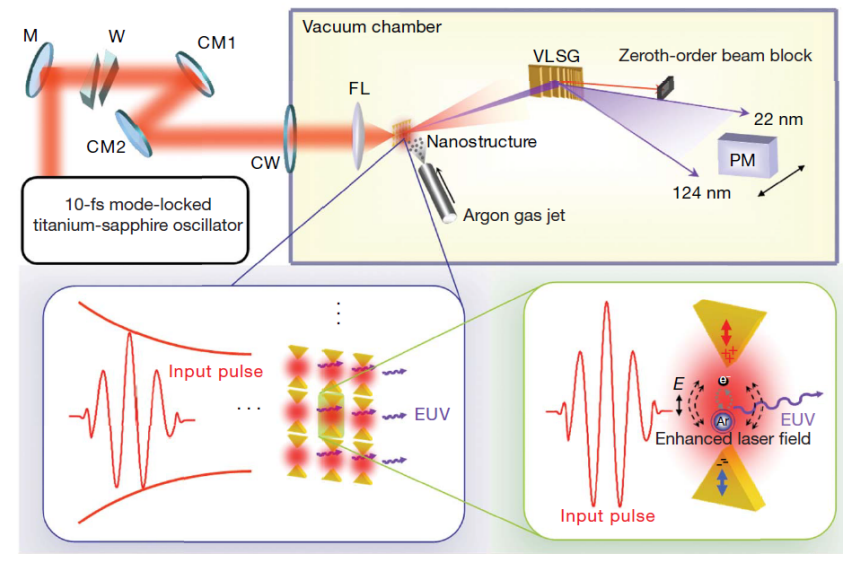

(a) Experimental scheme for plasmon-enhanced HHG

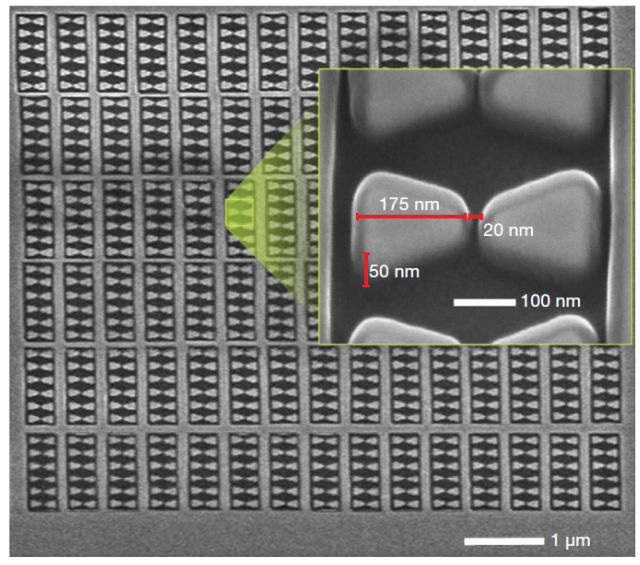

(b) SEM image of bow-tie nanoantennas

Figure 1.1: Experimental setup and nanostructures for plasmon-enhanced HHG. (a) Ultrashort, low-energy laser pulses from a Ti:sapphire oscillator are enhanced in bow-tie nanoantennas and the excitation of noble gas atoms is induced in the associated near-fields. EUV radiation is spectrally resolved and recorded with a scanning photomultiplier detector. The experiment was carried out under high vacuum conditions. (b) Scanning electron micrograph of a bow-tie nanoantenna array used for field enhancement. Images taken from Ref. [24]. (c) 2008 npg. 
regions between the triangular tips. Although the incident laser peak-intensity of about $10^{11} \mathrm{~W} / \mathrm{cm}^{2}$ is not sufficient to trigger HHG, it was believed that the near-field intensity enhancement of at least two orders of magnitude would allow for HHG in argon atoms delivered to the nanostructures by a gas nozzle. In this experiment, Kim et al. observed EUV emission down to a wavelength of about $50 \mathrm{~nm}$. Figure 1.2 shows the reported spectrum taken from their 2008 publication. The wavelength positions of the EUV emission peaks appear to fit perfectly to the odd overtones of the driving $800 \mathrm{~nm}$ laser light and, therefore, were thought to correspond to coherent high harmonic radiation up to the $17^{\text {th }}$ order. The remarkably high photon flux reported in their paper ( $>10^{8}$ photons per second)

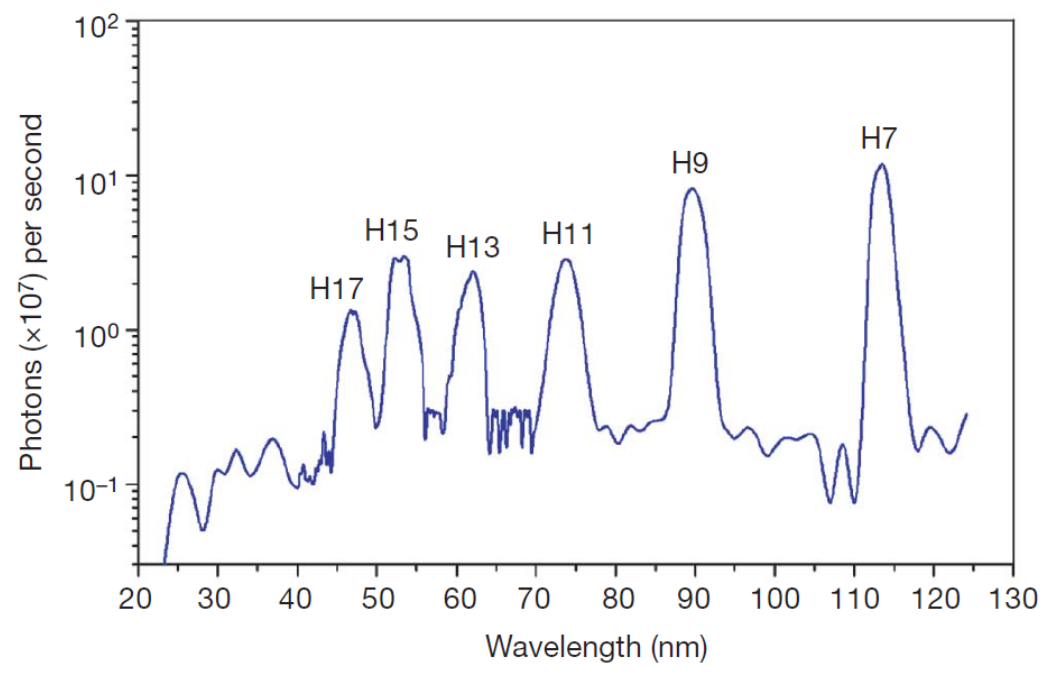

Figure 1.2: Extreme-ultraviolet emission spectrum. The EUV emission peaks are attributed to odd harmonics from the $7^{\text {th }}$ to the $17^{\text {th }}$ order. Graph is taken from Ref. [24]. (C) $2008 \mathrm{npg}$

suggested conversion efficiencies comparable to those reported for state-of-the-art HHG methods using amplified laser pulses in gas jets and capillaries. Thus, nanostructureenhanced HHG appear to offer a convenient method to generate bright, coherent EUV radiation from compact sources. If correct, such sources could provide attosecond pulses at high repetition rates for ultrafast comb spectroscopy. Indeed, it was thought that these groundbreaking results presented "an easier route to high harmony", as stated in a "News and Views" article [25] accompanying the first report of nanostructure-enhanced HHG. As a result, this work rapidly triggered significant excitement in the strong-field and nanooptics communities, with over 500 citations $^{1}$ to date.

\footnotetext{
${ }^{1}$ Google Scholar, http://scholar.google.de, January 24, 2014
} 
However, despite worldwide efforts from at least a dozen expert groups, these observations of nanostructure-enhanced HHG were not reproduced. The sole exceptions were followup reports of enhanced HHG in a different kind of nanostructure from the same research group [26, 27]. The experimental scheme for this second approach is illustrated in Figure 1.3(a). A hollow waveguide is utilized to enhance the incident low-energy laser pulses, resulting in local intensities sufficient to trigger HHG in a xenon gas volume at the tapered end of the waveguide close to a nanometric exit aperture. Figure 1.3(b) shows the published EUV spectrum for this experiment. Compared to the previous bow-tie measurements, the authors claimed to observe about an order of magnitude increase in photon flux and, additionally, an extension of the wavelength cutoff up to the $43^{\text {rd }}$ harmonic order.

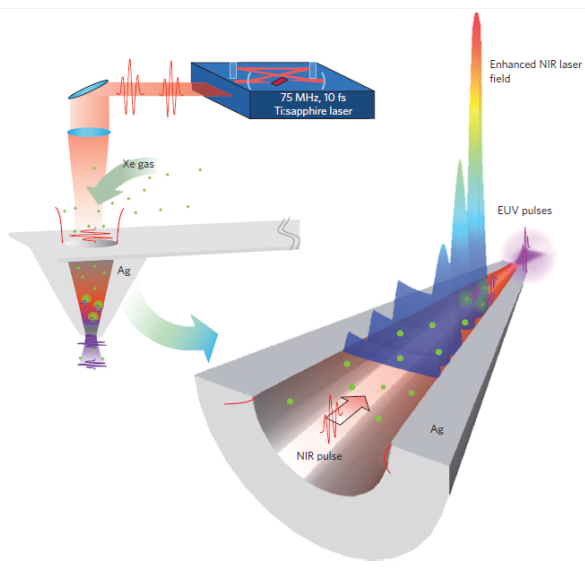

(a) Scheme for plasmon-enhanced HHG in hollow tapered waveguides

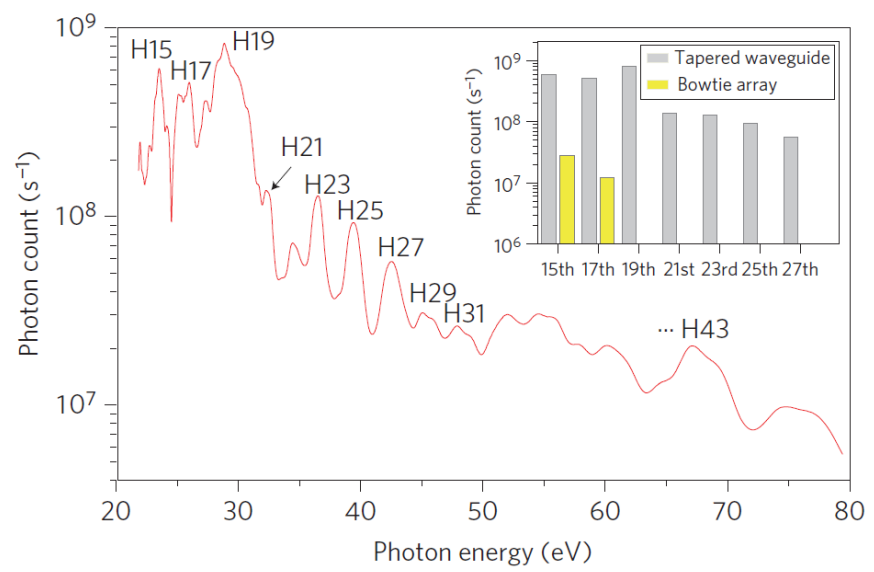

(b) EUV spectrum

Figure 1.3: Schematic illustration (a) and spectral data (b) for plasmon-enhanced HHG experiments using a hollow waveguide for field enhancement. Published by Park et al. in 2011. Images taken from Ref. [26].(C) 2011 npg.

The present thesis critically revisits EUV light generation processes in plasmonic nanostructures. It is shown that the concept of plasmon-enhanced HHG, as reported in Refs. [24, $26,27]$ is fundamentally flawed. Contrary to previous interpretation, our new experiments and analysis reveal a different origin of the EUV emission seen under these conditions and clearly show that it is in fact not caused by coherent HHG. Instead, the radiation originates predominantly from incoherent fluorescence due to multiphoton and strong-field gas excitation and ionization. These findings beg an important question: Is nanostructure- 
enhanced HHG feasible at all under the reported conditions? It is of paramount importance to clarify this issue as quickly as possible and to identify new avenues for realizing nanostructure-assisted strong-field processes, such as EUV light generation.

This work contributes to a clarification of this issue and describes future possibilities for strong-field nano-optics by demonstrating novel effects in the EUV spectral range. These findings include the gauging of local fields in nanostructures with the intensitydependent spectral characteristics of atomic fluorescence, the generation of fifth-order harmonic radiation intrinsic to bare gold nanostructures and the formation of a noble gas EUV nanoplasma exhibiting a pronounced optical bistability in tapered waveguide structures.

\section{Outline}

This thesis comprises a compilation of three original publications. These articles capture the main parts of the conducted experiments and contain all considerations and conclusions concerning the generation of EUV light in resonant plasmonic nanoantennas and tapered hollow waveguides.

Chapter 2 reprints a Brief Communications Arising [28], which is the commentary format of the scientific journal Nature. This paper directly concerns the first report of nanostructure-enhanced HHG [24] and presents a short critical discussion of plasmonic EUV light generation in this context. For completeness, the accompanying reply of Kim et al. [29] is reprinted in the Appendix A. Due to the inherent length restriction of such commentary formats, the article in Chapter 2 only covers a small fraction of the results obtained with resonant plasmonic nanoantennas and briefly sketches the fundamental considerations concerning nanostructure-enhanced HHG.

In Chapter 3, a comprehensive article provides an extended presentation of our experiments with bow-tie nanoantennas and allows for a more detailed discussion of nanostructure-assisted EUV light generation. Furthermore, for the first time, the direct gauging of strong fields in plasmonic nanostructures and surface-enhanced fifth-order harmonic generation are reported.

The Letter reprinted in Chapter 4 presents an analysis of EUV light generation in tapered plasmonic waveguides, which is consistent with the findings in Chapters 2 and 3 concerning the feasibility of nanostructure-enhanced HHG. Moreover, the observation of an intensity-dependent hysteresis in the fluorescent EUV signal caused by the formation 
of a bistable noble gas plasma in the waveguide structures is discussed.

Before the results of this study are presented in Chapters 2-4, additional background material is provided, since the space restrictions of the paper formats prohibit a more detailed treatment of the basic concepts in those individual publications. The following sections provide a brief introduction into the relevant aspects of the generation of EUV light in atomic gases by strong-field excitation, the field enhancement in plasmonic antennas and waveguide nanostructures, and the combination of these concepts. In Chapter 5, a general discussion finally concludes the findings of this study and gives an outlook for the future investigations in this field. 


\subsection{Extreme-ultraviolet light generation in atomic gases}

Ever since coherent laser radiation from mid-infrared to ultraviolet frequencies has been available, revolutionizing daily life and modern science, researchers have also sought for laser-like radiation with even shorter wavelengths.

Currently, the key approach to access wavelengths in the EUV and soft X-ray spectral regimes at laboratory scales is the conversion of extremely intense visible or infrared laser radiation in gaseous media. Two distinct processes far beyond the realm of linear optics are responsible for the generation of EUV light in gas atoms. Specifically, ultrashort, highenergy laser pulses can produce highly-energetic photons via multiphoton and strongfield excitation of gas atoms accompanied by either fluorescence or high-order harmonic generation. These two processes have fundamental differences in terms of their excitation schemes and the optical properties of the produced radiation. In the following, the basic concepts of EUV fluorescence and HHG are briefly presented.

EUV fluorescence Fluorescent light emission from gas atoms or ions is caused by the transition of excited electrons from higher-energetic states to lower-lying states. The characteristic energy of a particular fluorescent photon is therefore determined by the energy difference of the involved states, and this amount of energy is at least required to excite the system in advance. In the case of EUV fluorescence, the corresponding energies are on the order of several tens of electron volts $(\mathrm{eV})$, and an excitation with infrared light frequencies (photon energies below $2 \mathrm{eV}$ ) implies that several photons have to be absorbed simultaneously to trigger the process. ${ }^{2}$ Due to the very large number of infrared-photons required for such a multiphoton excitation, this process is only feasible at high laser intensities exceeding $10^{13} \mathrm{~W} / \mathrm{cm}^{2}$. Fig. 1.4 schematically depicts the nonlinear multiphoton process. Whereas the energy of a single infrared-photon (red arrows in Fig. 1.4(a)) is far too small to lift one ground-state electron into a higher-lying state, the simultaneous absorption of many photons is sufficient for such an excitation. After a certain lifetime in this excited state, the electron returns back to its initial ground-state through the emission of an EUV photon (see Fig. 1.4(b)). As illustrated in Fig. 1.4(c), atoms can be ionized if the number of photons, and thus the total energy absorbed by the electron, is large

\footnotetext{
${ }^{2}$ Please note that EUV fluorescence can also be triggered by considering other excitation methods, such as high-energy electron impact or gas discharges, which cause the ignition of a plasma. However, such excitation mechanisms will not be discussed here.
} 
enough to overcome the vacuum energy level $E_{v a c}$ of the atom. In turn, these ions can be excited in the same manner as the neutral atoms, acting as sources for fluorescence at different emission wavelengths due to their modified energy levels. Under certain excitation conditions - at high gas pressures or laser intensities-large free electron densities in the gas volume can lead to the formation of a laser induced plasma, additionally contributing to the generation of fluorescent emission [30, 31].

(a)

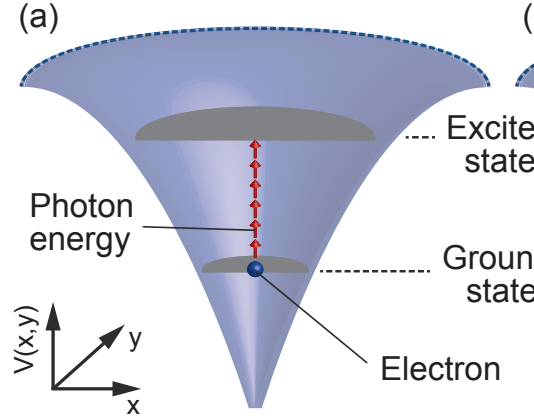

(b)

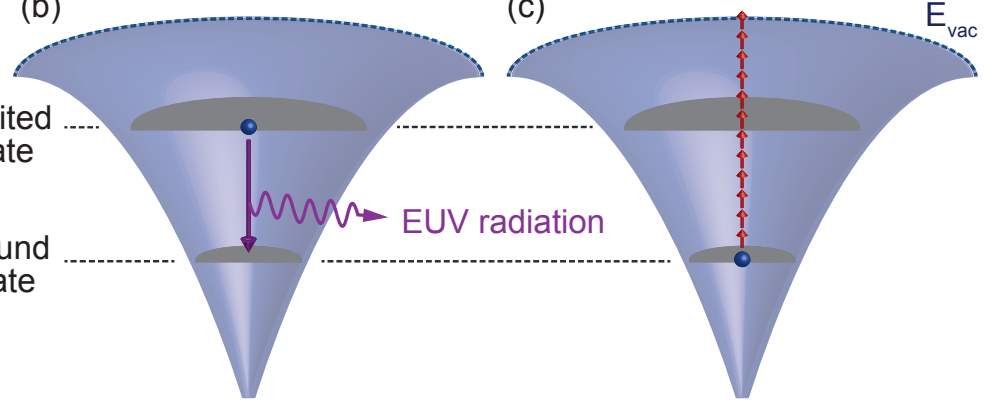

Figure 1.4: Schematic illustration of the fluorescence excitation and radiation mechanisms. Shown is an atomic coulomb potential (blue shaded). (a) A ground state electron is excited into a higher-lying state via multiphoton absorption. (b) After a certain lifetime the excited electron decays back into the ground state under emission of an EUV photon. (c) Multiphoton ionization. $E_{v a c}$ displays the vacuum energy level.

Following the above explanation, it becomes clear that every gas species exhibits its characteristic fluorescence spectrum with emission lines corresponding to the specific transitions of the respective element. By way of example, Fig. 1.5 shows two typical EUV spectra for nitrogen (a) and argon (b), which were obtained using ultrashort infrared laser pulses for the excitation of the gas atoms [32].

The emission characteristics of the EUV fluorescence are determined by the nature of the generation process, which is intrinsically incoherent and governed by the spontaneous lifetime of the excited energy states. These properties cause the fluorescent photons to have random phase relationships and direction. Thus, the optical properties of the driving laser beam are lost, and the EUV emission is neither temporally nor spatially coherent and is not pulsed in time. Furthermore, the emission yield scales linearly with gas density and exhibits a nonlinear intensity dependence caused by the multiphoton or plasma excitation. 


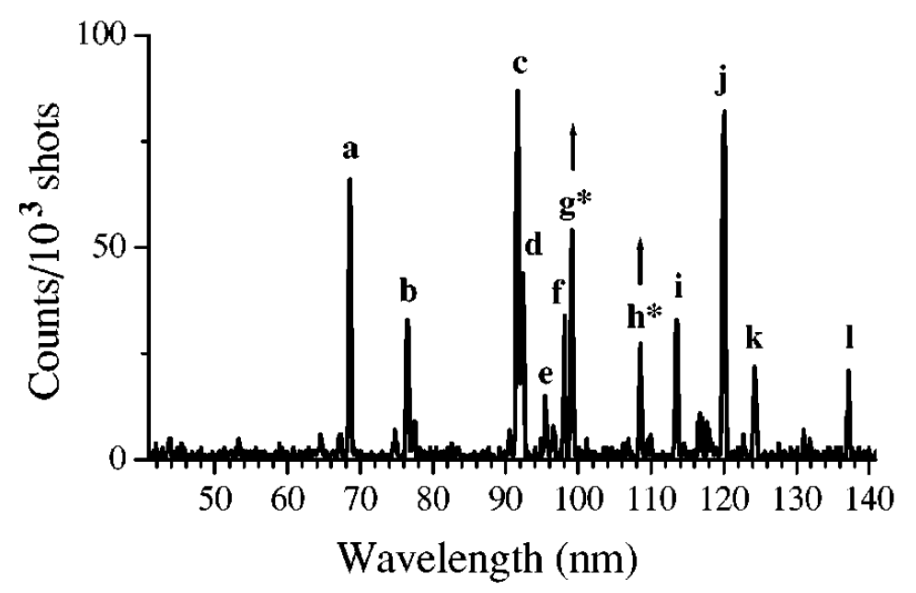

(a) Nitrogen spectrum

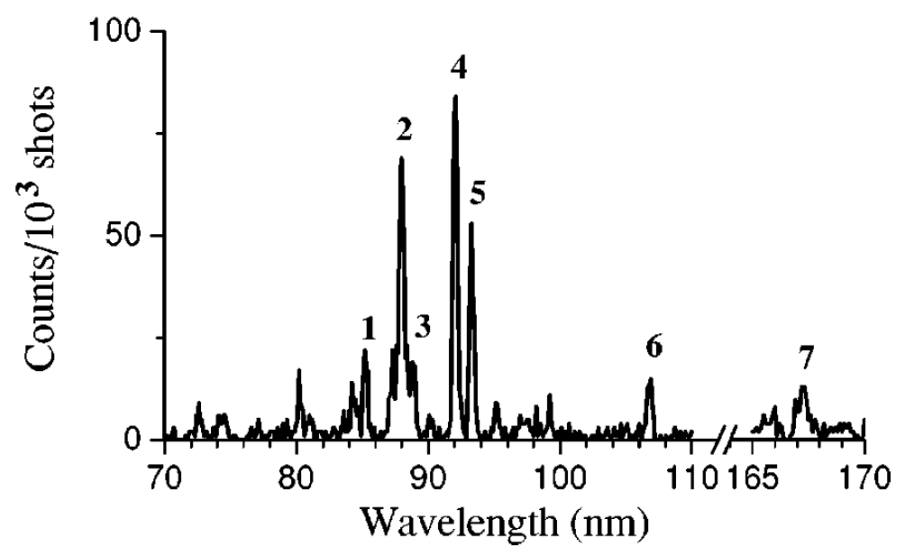

(b) Argon spectrum

Figure 1.5: EUV fluorescence spectra from nitrogen and argon gas. The fluorescence was excited with ultrashort infrared laser pulses at an intensity of $10^{14} \mathrm{~W} / \mathrm{cm}^{2}$. The gas pressure was 100 mtorr. Graphs taken from Ref. [32]. (c)2004 APS.

High harmonic generation In contrast to EUV fluorescence, high harmonic generation is a coherent process, which conserves the optical properties of the driving laser source. This facilitates the access to ultrashort, extreme-ultraviolet light pulses at laboratory scales $[33,34,35]$ and makes HHG valuable for novel experimental realizations such as attosecond spectroscopy [22,23] or nanoscale photonic imaging [21].

HHG is the frequency up-conversion of intense laser radiation in atomic gases and leads to the observation of odd overtones — harmonic orders — of the incident light frequency. Two excitation regimes attributed to below-threshold low-order harmonic generation and above-threshold high-order harmonic generation are distinguishable. Depending on the 
excitation intensity, harmonic orders with photon energies below the atomic ionization threshold are treated in an electron perturbation theory [36, 37], whereas the generation of above-threshold harmonic orders is usually explained in a three step picture (including electron re-collisions), called the simple man's model [20]. The following section focuses on the above-threshold excitation regime, since the main statements made in this work concern high-order harmonic generation.

The excitation scheme for above-threshold HHG (hereafter simply termed HHG) in noble gases completely differs from the mechanism for EUV fluorescence. Specifically, the HHG process can only be induced using ultrashort, linearly polarized laser pulses with high enough peak intensities to trigger tunnel-ionization of gas atoms. For noble gases and infrared light wavelengths, this situation corresponds to intensities in the excess of $10^{13} \mathrm{~W} / \mathrm{cm}^{2}$, which are usually reached using moderately focused femtosecond pulses from Ti:sapphire laser amplifiers.

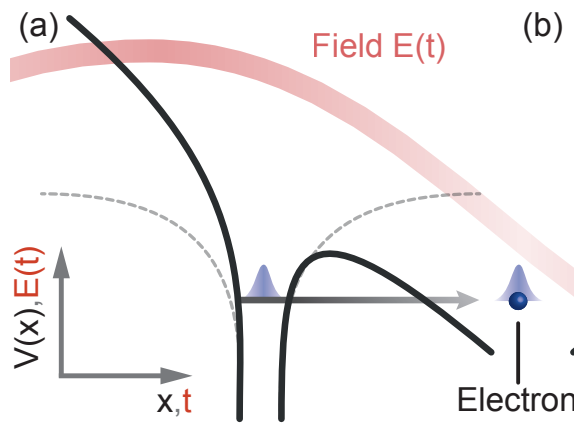

Tunnel ionization

(b)

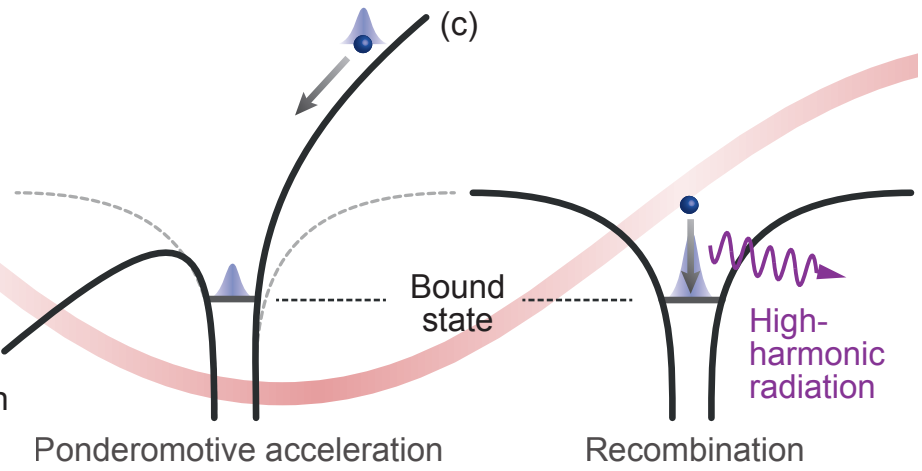

Figure 1.6: Three-step explanation of the simple man's model. (a) Field-ionization of an atomic system by tunneling of one electron through a short-lived finite potential-barrier. (b) Acceleration of the quasi-free electron in the oscillating laser field $E(t)$. (c) Re-collision of the electron with the parent ion core and emission of EUV light pulses.

A microscopic examination within the semi-classical simple man's model [20] leads to a coarse description of HHG without the need for a detailed quantum mechanical treatment $^{3}$. Figure 1.6 illustrates the particular sequences of the simple man's model within a half-cycle of the driving laser field (red curve). In the first step, one bound-state electron is liberated into the continuum via field driven-ionization, when the absolute field value is close to its maximum. At this point, the applied strong field alters the atomic Coulomb

\footnotetext{
${ }^{3}$ Of course, for an explicit treatment of $\mathrm{HHG}$, quantum mechanics have to be considered, e.g. for the calculation of the probability amplitudes of tunnel ionization and electron re-collisions [33].
} 
potential (solid black) relative to the undisturbed case (dashed gray), and the electron escapes by quantum mechanical tunneling through a short-lived finite potential-barrier. The quasi-free electron is then accelerated in the oscillating laser field and therefore gains additional ponderomotive energy, which is proportional to the intensity of the driving laser field. Finally, the electron returns to the starting point and re-collides with the ion under the emission of highly-energetic photons with discrete frequencies corresponding to odd overtones (harmonics) of the driving wave. The maximum energy $E_{\max }$ of the photons and the corresponding maximum frequency $\omega_{\max }$ of the harmonics generated in this scheme is determined by the cut-off law [20, 38]

$$
E_{\max }=h \omega_{\max }=E_{v a c}+3.17 U_{p} .
$$

Here, $E_{v a c}$ is the ionization energy of the atoms, $U_{p} \propto I \omega_{0}^{-2}$ represents the ponderomotive potential $^{4}$, and the second term determines the maximum kinetic energy $\left(3.17 U_{p}\right)$ that an electron can have upon re-collision [20]. The cut-off law in equation 1.1 shows that the possible photon energies for HHG scale linearly with the laser intensity and are not determined by any atomic energy transitions. Hence, in contrast to EUV fluorescence, the high harmonic emission from any gas species exhibits very similar spectral shapes, which consist of odd harmonics of the fundamental laser frequency. The exhibited emission strengths and the maximum cut-off energies are influenced by the atomic properties of the respective gas species and the excitation intensities.

As an example, Figure 1.7 reprints one of the first HHG spectra reported in Ref. [19]. In this early experiment, a xenon gas jet was excited with amplified picosecond laser pulses at $1064 \mathrm{~nm}$ central wavelength. The reported peak intensity of the laser pulses was $3 \times 10^{13} \mathrm{~W} / \mathrm{cm}^{2}$, and the gas pressure was 15 torr. The spectrum shows odd harmonics of the incident laser radiation spanning from the $9^{\text {th }}$ to the $21^{\text {st }}$ order. Higher orders are not observed due to the energy cut-off presented in equation 1.1.

Beyond these unique spectral characteristics, it may be noted that HHG is generally a rather inefficient process [38]. In the single atom picture of the simple man's model, this inefficiency is caused by the very low re-collision probability of the electrons with their parent ions. In general, sufficient photon fluxes are only obtainable by considering the coherent buildup of the harmonic radiation over a large propagation distance in

\footnotetext{
${ }^{4}$ The ponderomotive potential describes the mean energy, that an electron can gain in an oscillating electromagnetic field with intensity $I$ and frequency $\omega_{0}$.
} 


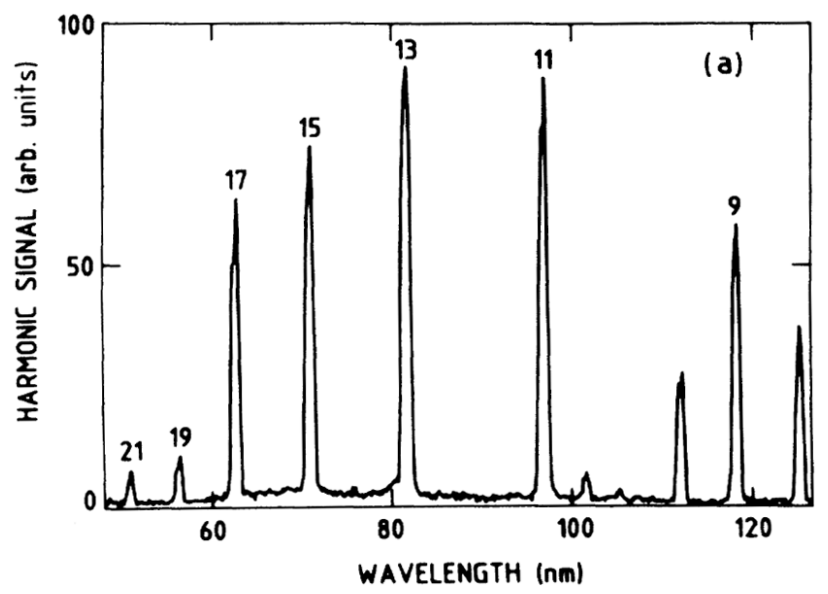

Figure 1.7: High-order harmonic spectrum. One of the first demonstrations of HHG in a xenon gas jet by using $1064 \mathrm{~nm}$, picosecond laser pulses. Graph is taken from Ref. [19]. (C)1989 APS.

a mesoscopic (tens to hundreds of micrometers or even millimeters) generation volume. Therefore, the high harmonic photon flux scales quadratically with the number of participating atoms, as shown in Fig. 1.8. However, such a coherent radiation buildup is only obtainable under ideal conditions that are influenced by certain propagation effects of the radiation during the generation process. In this respect, the most important propagation effect is the dephasing between the driving and the generated radiation due to frequencydependent dispersion in the gas volume, whereas effects like absorption or defocusing are usually negligible for high harmonic wavelengths [33]. The two main contributions to the phase mismatch are plasma dephasing and geometrical dephasing caused by the free electrons (plasma dispersion) in the ionized gas volume and the focusing conditions for the driving laser beam (Gouy-phase shift), respectively. Both kinds of dephasing lead to a mismatch of the phases between the driving laser wave and the high harmonic waves during the propagation in the gas volume, resulting in destructive interference for the high harmonic radiation when the phase difference reaches $\pi$. The corresponding limited propagation distance for coherent buildup of the high harmonic radiation is determined by the coherence lengths $L_{g, f s}$ and $L_{f e}$ for the geometrical and the plasma dephasing, respectively [33]:

$$
\begin{gathered}
L_{g, f s}=\frac{\pi z_{0}}{N} . \\
L_{f e}=\frac{2 \pi c \omega_{0}}{\omega_{p}(\tau)^{2} N} .
\end{gathered}
$$


Here, $N$ is the order of the harmonic radiation, $z_{0}$ is the Rayleigh length of the laser focus, and $\omega_{0}$ and $\omega_{p}(\tau)$ are the light and the plasma frequencies, respectively. Maximizing these coherence lengths by choosing the proper focusing conditions and gas pressures yields the only means of generating appreciable high harmonic radiation.

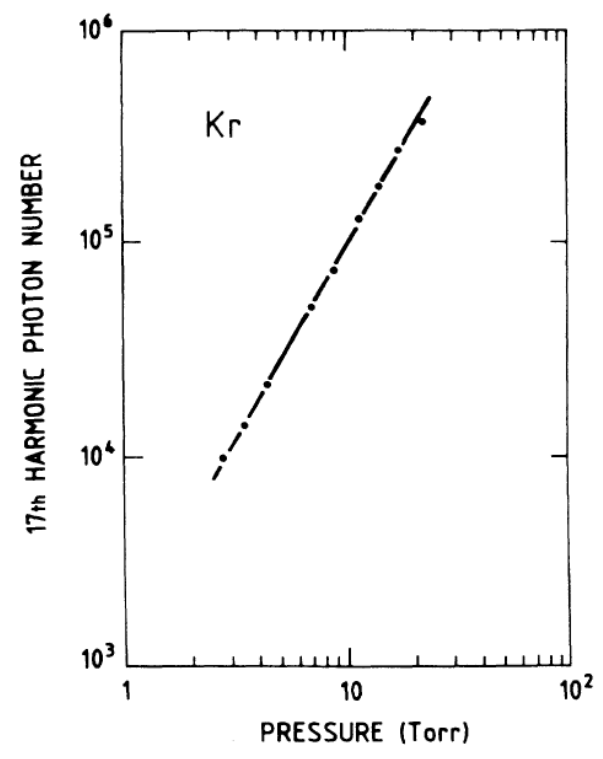

Figure 1.8: Quadratic scaling of the harmonic yield with target density. The doublelogarithmic plot shows the photon numbers for the $17^{\text {th }}$ harmonic order from krypton as a function of gas pressure. The slope of 2 indicates the quadratic pressure dependence of the high harmonic generation process. This measurement was carried out under phase-matched conditions. Graph is taken from Ref. [19]. (C)1989 APS.

For the discussion below, it is important to stress that under ideal-perfectly phasematched - conditions the high harmonic output power scales quadratically with the number of participating gas atoms in propagation direction or, more generally, with the pressurelength product.

\subsection{Plasmonic nanostructures for field enhancement}

Plasmonic light concentration into sub-wavelength volumes allows for orders of magnitude enhancements of incident fields. This familiar effect relies on the optical properties of the material that makes up the nanostructures. For visible and infrared excitation frequencies, gold and silver are plasmonic metals and are harnessed in numerous nanostructurebased applications. The negative real part and positive but small imaginary part of the 
dielectric function $\varepsilon_{m}(\omega)$ enables the resonant excitation of the electron plasma at the surface of a plasmonic metal with an oscillating electromagnetic wave. A detailed theoretical treatment within classical electrodynamics (Maxwell's equations in conjunction with the Drude-model for metals) provides a good description of the associated plasmonic phenomena [39]. Depending on the shape of the nanostructures, two types of plasmonic effects are generally distinguishable.

First, the electron density oscillations at the surface of a planar metallic structure cause so-called surface plasmon polaritons (SPPs), which can propagate in the form of exponentially-decaying electromagnetic waves (confined in the $z$-direction) at the metal/dielectric interface (see Fig. 1.9(a)). Second, the collectively excited free electrons of a sub-wavelength metal particle lead to a resonantly oscillating electron density within the particle boundaries, termed localized surface plasmons (LSPs) (see Fig. 1.9(b)). LSPs lead to a dipole-like field distribution and the concentration of the fields at the edges of the nanoparticles 5 .

(a)

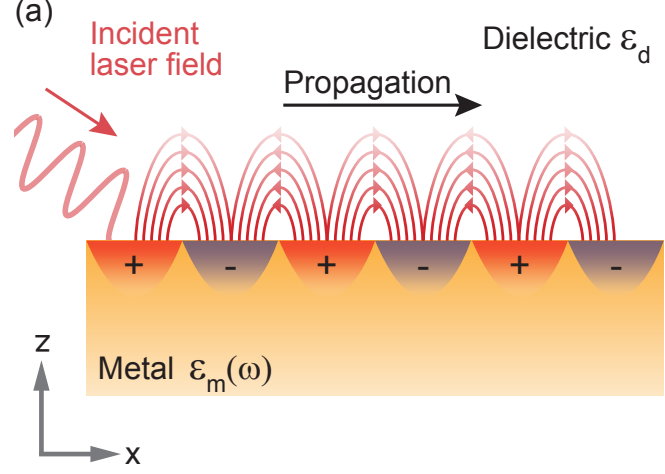

(b)

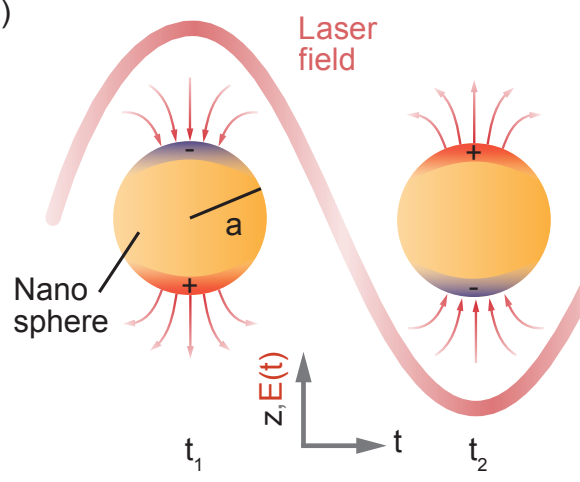

Figure 1.9: Illustrations of light induced excitation of a plasmonic metal. For a planar metal surface (a) and a sub-wavelength metal particle (b) both in the environment of a dielectric with constant permittivity $\varepsilon_{d}$. (a) An impinging laser field excites surface plasmon polariton waves (red arrows), which propagate in $x$-direction at the metal/dielectric interface. (b) Spherical nanoparticles exhibiting LSPs driven by an external oscillating field E(t) (red curve). The dipole-like field distribution outside the particles is indicated by the red arrows.

Both of these plasmonic effects can be utilized in a vast number of different nanostructure geometries for light localization. A few examples of such geometries include

\footnotetext{
${ }^{5}$ Please note that this explanation is only valid for particles which are much smaller that the light wavelength $\lambda$ (quasi-static approximation, see below). For larger particles ( $>100 \mathrm{~nm}$ for visible or infrared radiation) the Mie theory [40] has to be applied. However, a detailed explanation of this theory shall not be given here.
} 
nanoparticles, sharp tips, and even rough surfaces. Additionally, much more complex structures such as split ring resonators, diverse antennas, and hollow waveguides are also known. The manifold research activity in this topic has led to an impressively large number of reports $[9,10,11,41,42]$ and several textbooks [43, 39] about the optical properties of light-concentrating nanostructures and possible applications. Due to the tremendous variety of imaginable geometries and effects, it is not in the scope of this thesis to give a complete overview of the whole field of plasmonics. Instead, the most important aspects of the plasmonic excitation mechanisms for bow-tie nanoantennas and tapered hollow waveguides - the structures utilized in the present work - are elucidated in the following two paragraphs.

Resonant bow-tie antennas Generally, metal antennas offer the important possibility to amplify electromagnetic radiation, whether for radio or light frequencies, and allow for high field enhancements due to their adaptability in terms of tailored shapes. In this respect, the most important property comprises the antenna's resonance frequency at which efficient operation can be realized. For light-enhancing nanoantennas, this frequency is determined by the localized surface plasmon resonance, which is shape and material dependent. The simplest expression for such a LSP resonance can be found for a metal sphere of radius $a$, excited with an oscillating light field (see Fig. 1.9(b)). If the radius $a$ is much smaller than the light wavelength $\lambda$, the field distribution in the metal can be considered quasi-static, and this approximation allows us to derive the sphere's electric polarizability [39]

$$
\alpha=4 \pi a^{3} \frac{\varepsilon_{m}(\omega)-\varepsilon_{d}}{\varepsilon_{m}(\omega)+2 \varepsilon_{d}} .
$$

Here, $\varepsilon_{d}$ is the permittivity of the surrounding dielectric medium, and $\varepsilon_{m}(\omega)$ is the frequency-dependent dielectric function of the metal. For a plasmonic Drude metal, the polarizability $\alpha$ exhibits a strong resonance with a resonance frequency independent of radius (see Fig. 1.10, solid blue). The situation changes for the same analytic treatment of an ellipsoid with different semiaxes $a_{1} \leq a_{2} \leq a_{3}$. The quasi-static approximation leads to

$$
\alpha_{i}=4 \pi a_{1} a_{2} a_{3} \frac{\varepsilon_{m}(\omega)-\varepsilon_{d}}{3 \varepsilon_{d}+3 L_{i}\left(\varepsilon_{m}(\omega)-\varepsilon_{d}\right)}
$$

as an expression for the polarizabilities $\alpha_{i}(i=1,2,3)$ along the major axes of the ellipsoid [39]. Here, $L_{i}=L_{i}\left(a_{i}\right) \propto a_{i}^{-2}$ is the axis-dependent geometry parameter, which has 
to satisfy the condition $\sum L_{i}=1$. For a sphere $L_{1}=L_{2}=L_{3}=1 / 3$, and equation 1.4 is recovered.

Equation 1.5 shows that the polarizability for an ellipsoid exhibits a shape-dependent resonance for an excitation along the respective axis. This enables us to tune the frequency of an antenna by tailoring its shape. For example, an antenna-like ellipsoid, i.e., with a high aspect ratio of the axis $\left(L_{1}<<L_{2}=L_{3}\right)$, exhibits a resonance frequency (of the long axis) that is significantly red shifted compared to the frequency of a sphere (see Fig. 1.10).

Figure 1.10: Absolute value of the polarizability $|\alpha|$ for a small gold sphere and a gold ellipsoid with high aspect ratio. Shown are the calculated polarizabilities for a sphere and an antenna-like ellipsoid with respect to the excitation frequency of the driving light wave (expressed in $\mathrm{eV})$. The dielectric function $\varepsilon_{A u}(\omega)$ was fitted with an analytic model for the optical properties of gold [44].

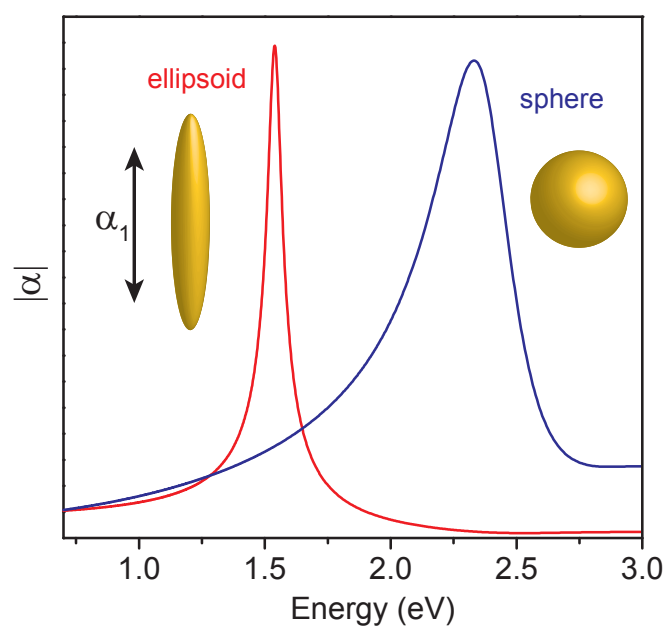

However, this above analytic treatment is only valid for the quasi-static approximation, i.e., if the dimensions of the particles are significantly smaller than the excitation wavelength. Nevertheless, it already shows the great potential of tailored nanoantennas to reach high field enhancements. Furthermore, beyond the quasi-static approximation, diverse effects, e.g., interband transitions in the respective metals, influence the shape and width of the exhibited plasmon resonance. For larger antennas with complicated shapes, such as those shown schematically in Figure 1.11(a), the description via an analytic model is quite difficult, and numerical simulation methods based on the solution of Maxwell's equations have to be applied to calculate their optical properties. Specifically, for bow-tie antennas, the resonance frequency and also the field enhancement are critically dependent on the particular shape and can be tuned by the variation of the length $h$, opening angle $\theta$, thickness $t$, and gap size $d$, as well as the choice of the substrate for the bow-tie fabrication $[45,42]$.

The coupled dipole fields of two bow-tie elements in the tip-to-tip arrangement with 20 


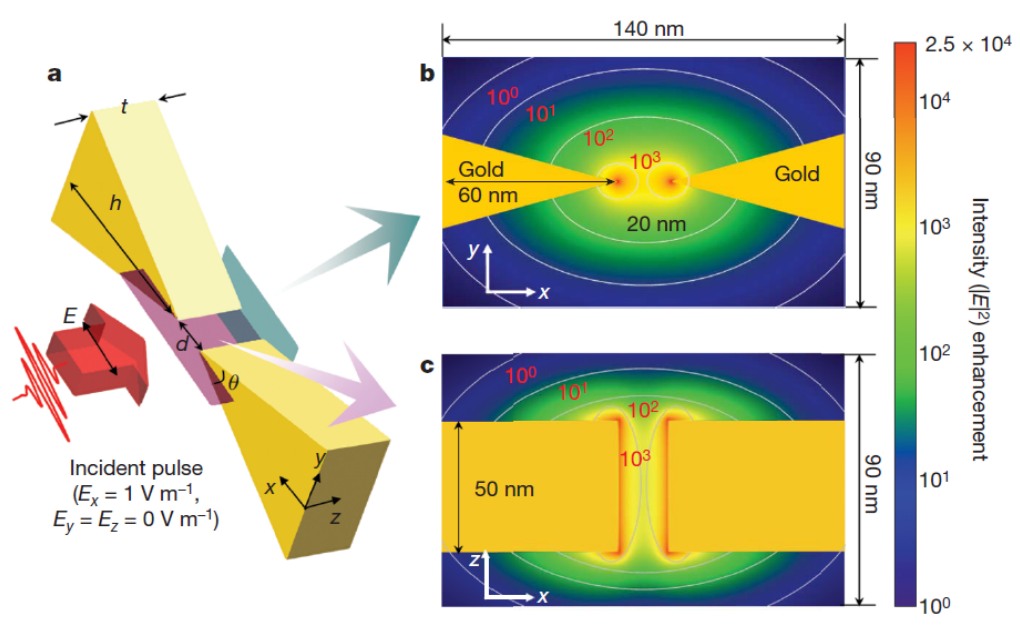

Figure 1.11: Scheme of a bow-tie antenna and FDTD simulations. (a) Schematic for the excitation for a bow-tie antenna pair with linearly polarized laser pulses. (b,c) FDTD simulations for the intensity enhancement. Images are taken from Ref. [24]. (c)2008 npg.

$\mathrm{nm}$ gap size are expected to result in an intensity enhancement of more than $10^{3}$ (corresponding to field-enhancement factors over 30) as shown theoretically in FDTD 6 simulations (see Figs. 1.11(b) and (c)) [24]. Even though these simulations, which are presented in Ref. [24], may overestimate the field enhancement by assuming ideal bow-ties with very sharp apexes, alternative simulations for realistically-shaped antennas still predict intensity enhancement factors of several hundreds [46].

Tapered hollow waveguides In contrast to plasmonic antennas, the excitation scheme for tapered hollow waveguides is yet somewhat unclear, and a fully established theoretical treatment is not available so far. ${ }^{7}$ Most likely, surface plasmon polaritons are induced at the inner walls of the hollow waveguide by an incident, linearly polarized light field and propagate towards the narrowed end of the taper (see Figs. 1.9(a) and 1.12). The SPP

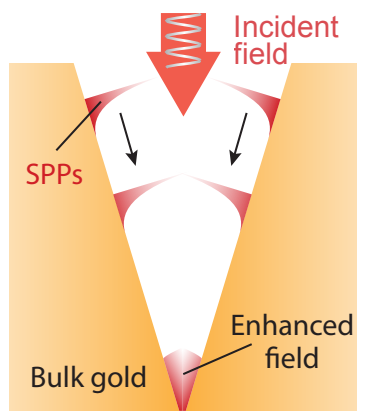

Figure 1.12: Waveguide excitation. drop below the light wavelength; this coupling results in a large field-enhanced volume near the exit aperture.

\footnotetext{
${ }^{6}$ Finite difference time-domain

${ }^{7}$ Please note that a first approach to quantify the field enhancement in tapered hollow waveguides involving numerical FDTD simulations was reported in Ref. [26] (see below).
} 
This possible excitation scheme is analogous to that of a solid metal taper, where the SPPs are induced at the outer surface and propagate to the apex of the tips $[47,48]$. However, in such a scheme, the direct plasmonic excitation of the plain metal surface is restricted due to the dispersion relation of the SPPs. Considering the interface between a metal with frequency-dependent dielectric function $\varepsilon_{m}(\omega)$ and a dielectric medium with a constant $\varepsilon_{d}$, the wave vector $\beta$ of a SPP can be expressed as [39]

$$
\beta=k_{0} \sqrt{\frac{\varepsilon_{m}(\omega) \varepsilon_{d}}{\varepsilon_{m}(\omega)+\varepsilon_{d}}}
$$

Here, $k_{0}$ is the wave vector of the exciting light wave. This dispersion relation causes a mismatch between the wave vectors of the incident light and the SPPs and, therefore, prohibits a direct excitation of the free electrons at the planar surface of the metal. Hence,

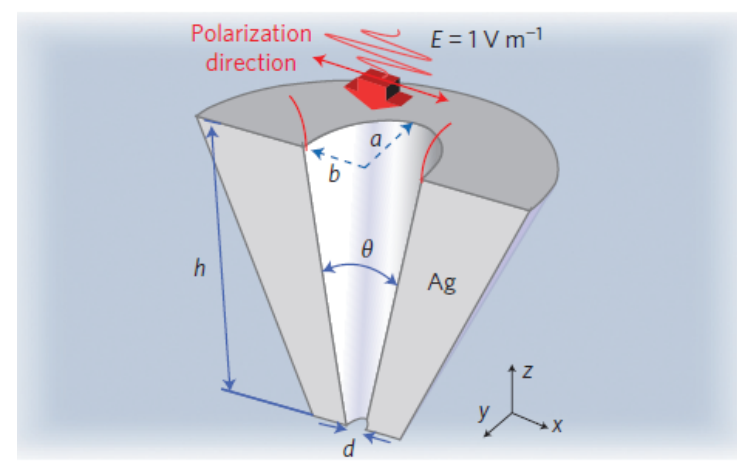

(a) Schematic of a hollow waveguide

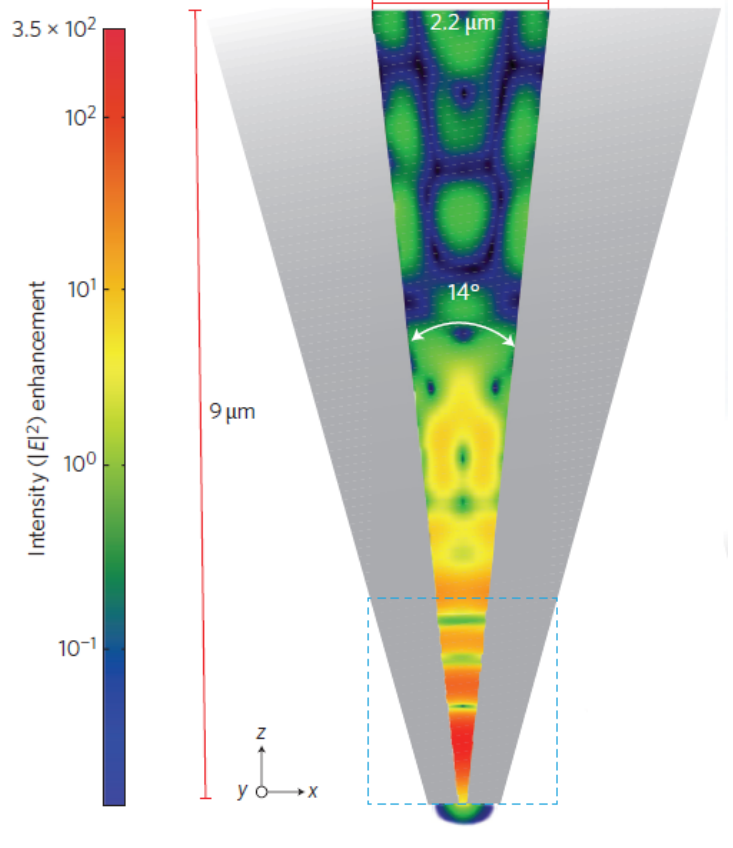

(b) FDTD simulations

Figure 1.13: FDTD simulations for intensity enhancement in a tapered hollow waveguide. (a) Schematic for the excitation of a hollow tapered waveguide with linearly polarized laser pulses. (b) FDTD calculations for the intensity enhancement within an elliptical waveguide structure. Images taken from Ref. [26]. (C) 2011 npg. 
for reasons of momentum conservation it is usually unavoidable to use a prism or grating to realize efficient SPP coupling in such a scenario [47, 48]. In contrast, the incoupling of SPP waves into a hollow tapered waveguide also seems to work without special structural requirements at its inner surface. Although it can be assumed that fabrication imperfections such as steps or roughnesses of the inner walls enable an excitation, the exact incoupling mechanism for SPPs is yet to be revealed. Besides these fundamental considerations, which surely demand further experimental investigations and the need for a profound theoretical model, numerical simulations have shown that high field enhancements are most likely possible in tapered hollow waveguides. Fig. 1.13 reprints the results of FDTD calculations from Ref. [26] for a silver waveguide. Here, intensity enhancement factors as high as 350 within the last $450 \mathrm{~nm}$ of the hollow taper are predicted. It is further reported that the right choice of specific geometrical parameters like height $h$, opening angle $\theta$, and entrance and exit aperture dimensions strongly influence the exhibited field enhancement, with the highest enhancement values expected for elliptically-shaped waveguides [26].

\subsection{Plasmon-enhanced strong-field gas excitation}

This section illustrates the translation of the strong-field gas excitation concept, as discussed in section 1.1, into nanostructure-enhanced implementations utilizing plasmonic light concentration in bow-tie antennas and tapered hollow waveguides. As already mentioned above, the main requirement to trigger strong-field optical effects, specifically with respect to EUV fluorescence and HHG in atomic gases, are local intensities in the excess of $10^{13} \mathrm{~W} / \mathrm{cm}^{2}$ for infrared and visible excitation frequencies. For quite a long time, this particular strong-field intensity regime was reserved for implementations involving the use of high-energy ultrashort laser pulses from $\mathrm{kHz}$ amplifier laser systems. Now, the concept of plasmonic light localization within suitable nanostructures enables us to access the strong-field intensity regime by utilizing low-energy radiation. As shown in the last section, sufficient local intensities above $10^{13} \mathrm{~W} / \mathrm{cm}^{2}$ are generally reachable in particular nanostructure geometries for incident intensities of only $10^{11} \mathrm{~W} / \mathrm{cm}^{2}$. Figure 1.14 schematically depicts the concepts of plasmon-enhanced gas excitation in the nanostructure geometries used in this thesis. In particular, these are arrays of bow-tie antennas (a) and single tapered hollow waveguides (b), which are fabricated on transparent substrates and on gold bulk supports, respectively. In both approaches, linearly polarized laser pulses 
from a MHz Ti:sapphire oscillator are concentrated in certain hot spot regions (see insets of Figs. 1.14 (a) and (b)), and noble gas atoms are excited in these near-field volumes.

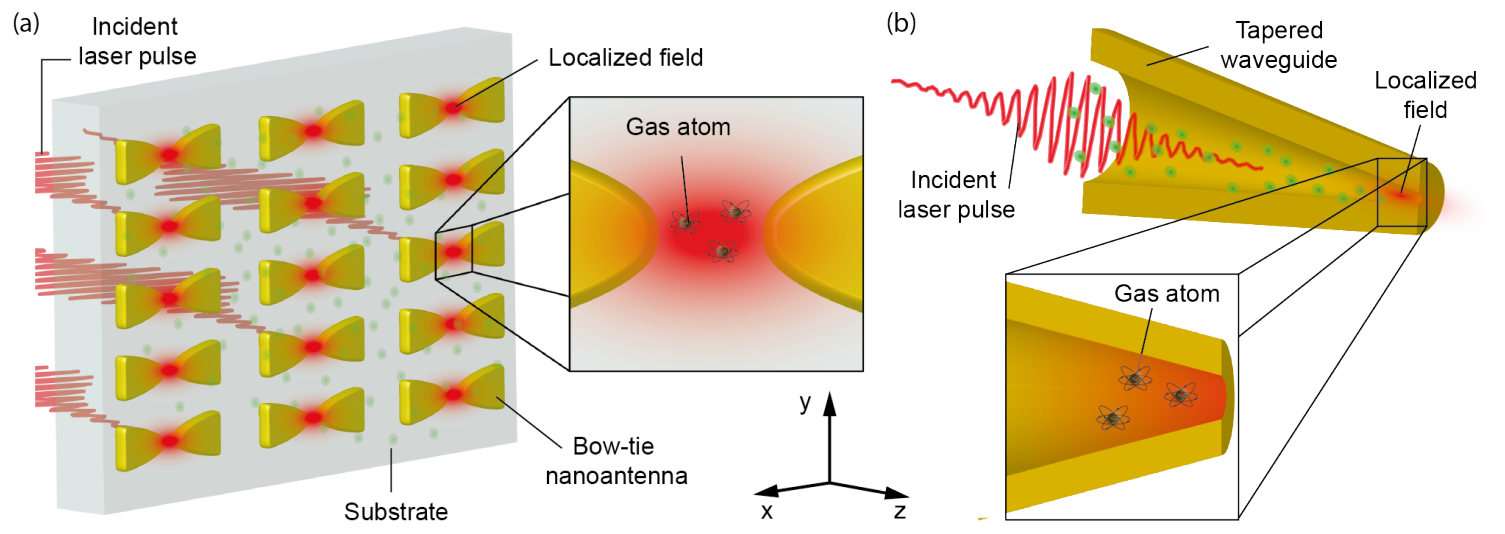

Figure 1.14: Schematic illustrations of the two concept for nanostructure-enhanced strongfield gas excitation. (a) An array of bow-tie nanoantennas is illuminated with ultrashort laser pulses. The local field enhancement of the incident light in the gap region (ca. $20 \times 20 \mathrm{~nm}^{2}$, see inset) leads to the excitation of the gas atoms exposed to the structures. (b) Laser pulses are introduced trough the entrance aperture of the tapered waveguide. The field enhancement in the last $500 \mathrm{~nm}$ of the waveguide results in the excitation of the gas atoms (see inset).

Whereas these concepts generally allow for the study of strong-field gas excitation and the resulting EUV emission, as shown below, it has to be pointed out that the total fieldenhanced volumes in both kinds of nanostructures are orders of magnitude smaller than those in implementations involving amplified laser pulses and large focal spots. This becomes already clear by considering just the generation lengths along the $z$-direction in Fig. 1.14, which are about $100 \mathrm{~nm}$ and $500 \mathrm{~nm}$ for the bow-tie antennas and the waveguides, respectively. In contrast, typical generation lengths for state-of-the-art HHG concepts are in the order of several millimeters or even centimeters [19, 49]. This specific aspect of the nanostructure-enhanced implementations is of great importance for the later discussion of the findings in this work. 


\subsection{Experimental methods}

Experimental setup The experimental setup implemented for this work, schematically shown in Fig. 1.15(a), consists of a vacuum generation chamber and EUV flat-field spectrometer, which are both evacuated separately. The setup allows for nanostructureenhanced gas excitation and EUV generation using low-energy laser pulses. For reference measurements, EUV light emission is also induced with high-energy laser pulses. Optical illumination with femtosecond laser pulses at a central wavelength of $800 \mathrm{~nm}$ is provided with a $78 \mathrm{MHz}$ Ti:sapphire oscillator and a $1 \mathrm{kHz}$ Ti:sapphire laser amplifier for the nanostructure-enhanced $(b, c)$ and the reference $(d)$ measurements, respectively. For

(a)

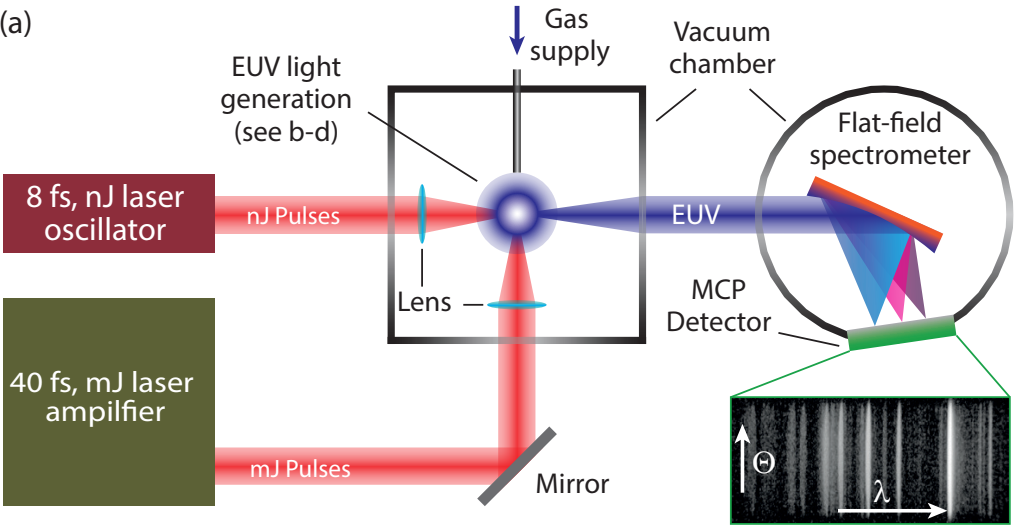

(b)

(c)
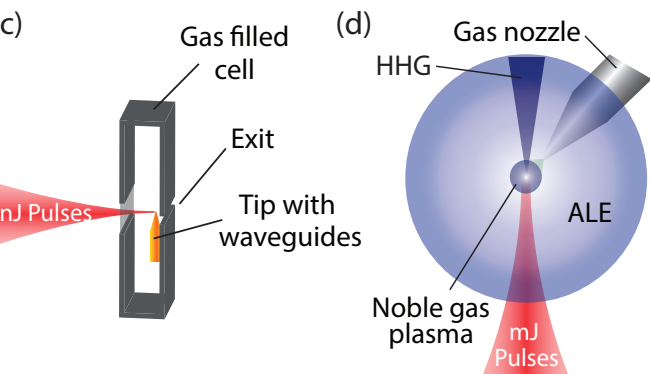

Figure 1.15: Experimental setup and excitation schemes for EUV light generation with and without nanostructure-enhancement. (a) Top view of the vacuum setup consisting of a generation chamber and an EUV flat-field spectrometer. The flat-field spectra (see inset, xenon fluorescence spectrum) are detected with a microchannel-plate detector (MCP) in combination with a CCD camera. b,c) Excitation schemes for the measurements using resonant bow-tie antennas (b) and tapered waveguides (c). Gas exposure of the bow-ties and the waveguides is realized with a nozzle and a purged gas cell, respectively. (d) Excitation scheme for the reference measurements using high-energy laser pulses. EUV fluorescence and high harmonic radiation are detected perpendicular and parallel to the laser beam direction, respectively. 
each of the measurements, EUV radiation is collected within a solid angle of $\pm 1.2^{\circ}$ and spectrally resolved with the EUV flat-field spectrometer. The spectra are detected with an imaging microchannel-plate (MCP) phosphor screen assembly and a CCD camera. As an example, a recorded flat-field image is shown in the inset of Fig. 1.15(a).

The excitation schemes for the nanostructure-enhanced measurements using arrays of bow-tie antennas and tapered hollow waveguides are depicted in Figs. 1.15(b) and (c), respectively. The oscillator pulses are focused tightly in both cases to reach incident intensities on the order of 0.1 to $1 \mathrm{TW} / \mathrm{cm}^{2}$. The plasmonic field enhancement in the nanostructures leads to orders of magnitude higher intensities in certain hot spots, and gas atoms exposed to these near-fields can be efficiently excited (see Fig. 1.14). For the bowtie measurements the gas atoms are provided via a nozzle facing the structures, while the hollow waveguides are placed in a purged gas cell with a small exit aperture for the collection of the generated EUV radiation. Measurements with amplified Ti:sapphire laser pulses in an ordinary gas jet (without nanostructure-enhancement) are performed to obtain reference EUV spectra. Fig. 1.15(d) illustrates the excitation scheme in the reference measurements, which employ the same nozzle as used in the bow-tie measurements. For comparison with the nanostructure-enhanced measurements, the fluorescent EUV emission perpendicular to the laser beam direction is recorded.

Home-built vacuum setup for EUV light generation and spectral detection The results presented in this thesis rely on the accurate analysis of EUV radiation with wavelengths well below 200 nanometers. In this wavelength regime, the absorption of photons in air under ambient conditions is a critical issue and limits photon propagation distances to the range of only several tens of micrometers ${ }^{8}$. Thus, for a practical experimental scheme in which EUV radiation from nanostructures can be investigated, it is necessary to implement the generation and the spectral detection of the EUV light in a suitable vacuum environment. For this purpose, one of the experimental efforts was the design and construction of a suitable vacuum setup. Figure 1.16(a) shows a technical drawing of the vacuum setup consisting of generation and spectrometer chambers. Under operation conditions, the chambers are separately evacuated with vacuum pumps to pressures well below $10^{-3}$ mbar and $10^{-5}$ mbar for the generation and spectrometer chamber, respectively (cf. Fig. 1.16(b)). Under these conditions, the mean free path of the EUV photons

\footnotetext{
${ }^{8}$ See X-ray database of the center for X-ray optics (CXRO, http://henke.lbl.gov/optical_constants/gastrn2.html, January 24, 2014).
} 
is sufficient $(>50 \mathrm{~cm})$ for the generated radiation to reach the detector.

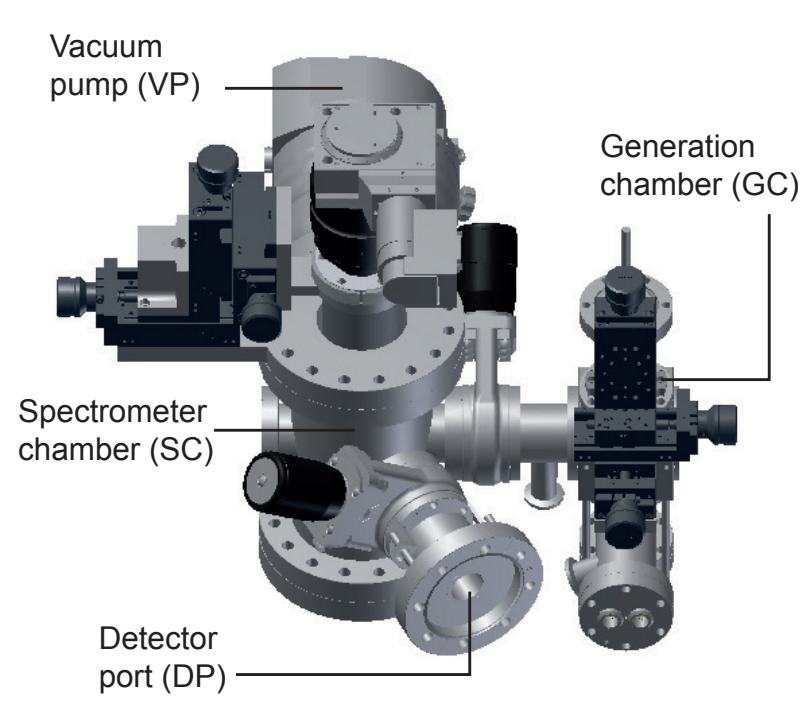

(a) CAD drawing of the vacuum setup

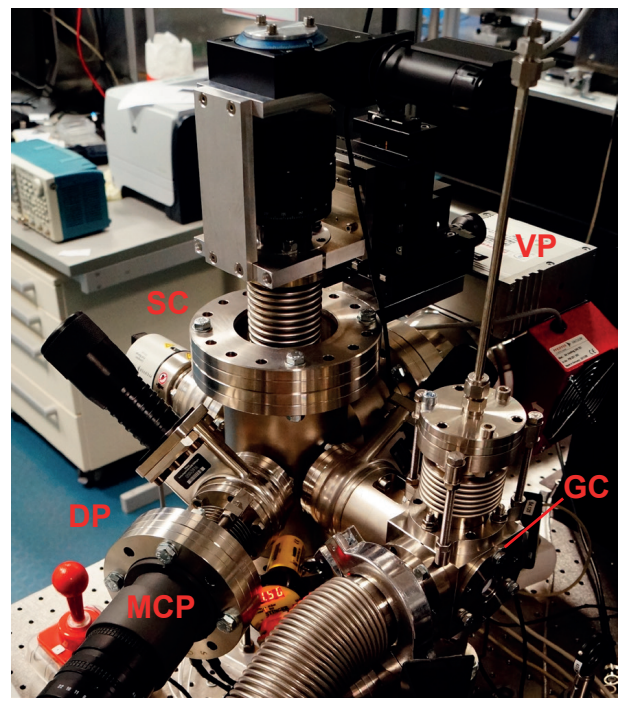

(b) Vacuum setup in operation

Figure 1.16: Home-built vacuum setup for EUV light generation and spectrally-resolved detection. (a) In the computer-aided design (CAD) drawing, the main components of the setup are illustrated. The generation chamber (GC) is equipped with all necessary tools to manage EUV light generation in a gas, with and without nanostructure-assistance. A spherical flat-field reflective grating inside in the spectrometer chamber (SC) focuses the emitted EUV radiation onto a virtual exit plane at the detector port (DP) and allows for a spectrally-resolved detection in a wavelength range of $30 \mathrm{~nm}$ to $500 \mathrm{~nm}$. (b) Fully connected vacuum setup under operation conditions. In contrast to the measurements with nanoantennas, in this case the gas supply (GS) is fixed, i. e., without manual translation, for the waveguide experiments.

Both parts of the vacuum setup are optimized for the excitation of noble gases with nanostructure-enhanced laser pulses and the spectral detection of the generated EUV light. In the generation chamber, micro-translation stages enable accurate positioning of the nanostructures relative to the laser beam, which is introduced through an entrance window and focused onto the structures either with an off-axis parabolic mirror or an aspherical lens. A variable gas supply is managed with a suitable feed-through in conjunction with a precise pressure adjustment. The detection of the EUV photons is realized with an EUV broadband reflective grating, which focuses a spectrally-resolved flat-field image of the EUV radiation onto the detector.

Nanostructure fabrication One of the primary challenges of this work is the fabrication of high quality plasmonic nanostructures, exhibiting large field enhancements and 
sufficient durability with respect to laser-induced thermal damage. Bow-tie antennas and tapered hollow waveguide nanostructures were fabricated on thin gold films and bulk gold material, respectively, using focused ion beam (FIB) lithography. In contrast to other fabrication techniques, such as electron-beam lithography, the direct milling of solids with accelerated ions has one striking advantage: the ability to change and control structural parameters during the production process. Hence, for the fabrication of prototype-like nanostructures, as employed in this work, FIB lithography presents the ideal method with respect to the optimization of the structural and optical properties.

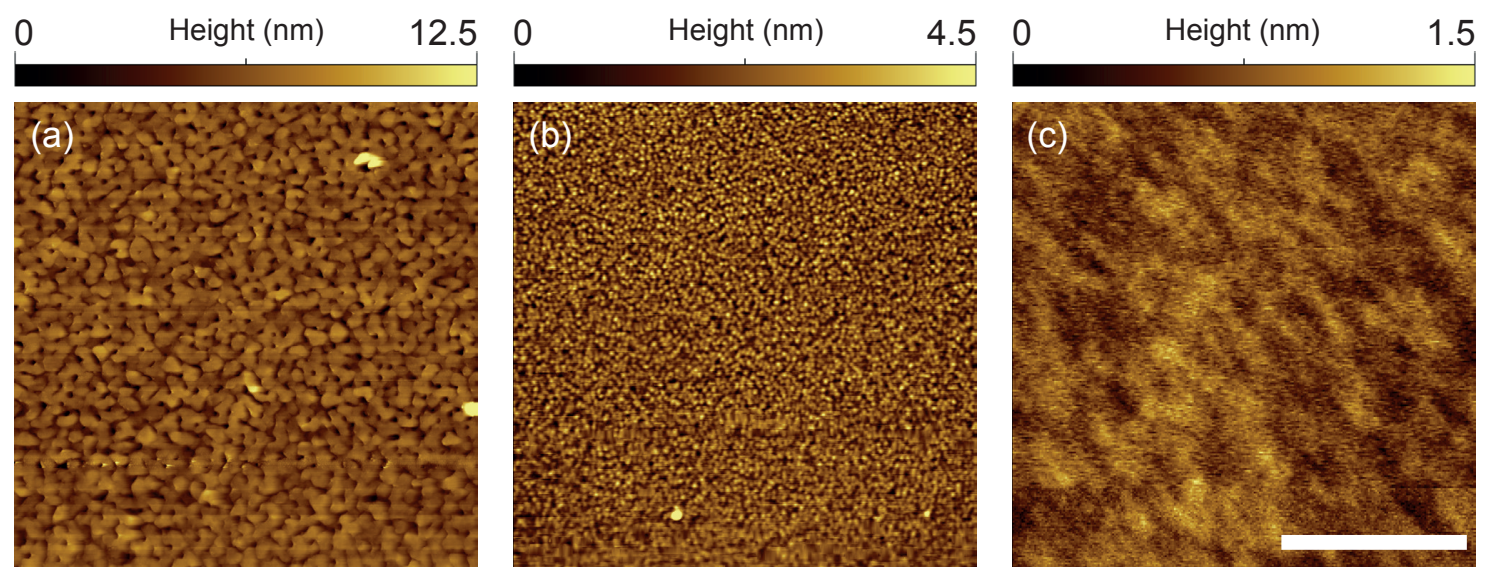

Figure 1.17: Optimization of the gold film roughness for the fabrication of nanoantennas with high structural quality. The three atomic force microscope (AFM) images show the different optimization stages with improving surface roughness from (a) to (c). The root-meansquared (RMS) value of the surface height within an area of $5 \mu \mathrm{m} \times 5 \mu \mathrm{m}$ has to be smaller than $1 \mathrm{~nm}$ to manage the fabrication of nanoantennas with sufficient structural quality. Thus, nanostructures on a rough gold film, as shown in (a) have a much lower quality than nominally identical structures on smoother gold films depicted in (b) and (c). The RMS heights are 1.6 $\mathrm{nm}, 0.7 \mathrm{~nm}$ and $0.2 \mathrm{~nm}$ for the surfaces shown in (a), (b) and (c), respectively. Scale bar: $2 \mu \mathrm{m}$.

Independent of the chosen preparation technique, the structural quality of the gold support strongly influences the exhibited field enhancement of the nanostructures. Specifically, in the case of bow-tie antennas, high quality structures were obtained by optimizing the surface roughness of the thin films on the dielectric substrates. Figure 1.17 depicts a series of atomic force micrographs for different gold thin films prepared in this work. The optimization of the evaporation conditions improved the surface roughness (measured by the root-mean-squared (RMS) value of the surface height) and enabled the fabrication of high quality nanoantennas. 


\title{
Chapter 2
}

\section{Nanostructure-enhanced atomic line emission}

\author{
M. Sivis, M. Duwe, B. Abel, and C. Ropers
}

Nature 485, E1 (2012)

DOI: $10.1038 /$ nature 10978

Plasmonic nanostructures offer unique possibilities for enhancing linear and nonlinear optical processes $[45,50,51,52,53,6]$. Recently, Kim et al. [24] reported nanostructure-enhanced high harmonic generation (HHG). Here, using nearly identical conditions, we demonstrate extreme-ultraviolet (EUV) emission from gas-exposed nanostructures, but come to entirely different conclusions: instead of HHG, we observe line emission of neutral and ionized gas atoms. We also discuss fundamental physical aspects limiting nanostructure-based HHG.

We conduct very similar experiments to those presented in ref. [24]. Specifically, bowtie nanostructure arrays are exposed to a noble-gas jet and illuminated with 8-fs laser pulses, and emitted radiation in the EUV is spectrally analysed (Fig. 2.1a). Further details and procedures are given in Methods. Figure 2.1b shows the raw detected spectral density of the first (solid black line) and second (solid red line) grating diffraction orders for an exemplary nanostructure (inset) and argon. We observe six main emission lines, some resolved into multiple lines in second order. All prominent features are attributed to atomic line emission of neutral and ionized argon $[54,55,56]$. Various optimized structures yield nearly identical spectra, whereas other gases display different transitions: Fig. 2.1c shows data using xenon on the same structures (Fig. 2.1b inset).

The presence of ionized atoms seems to support the feasibility of nanostructure-enhanced HHG. However, we have not observed any signature of HHG, even on increasing 

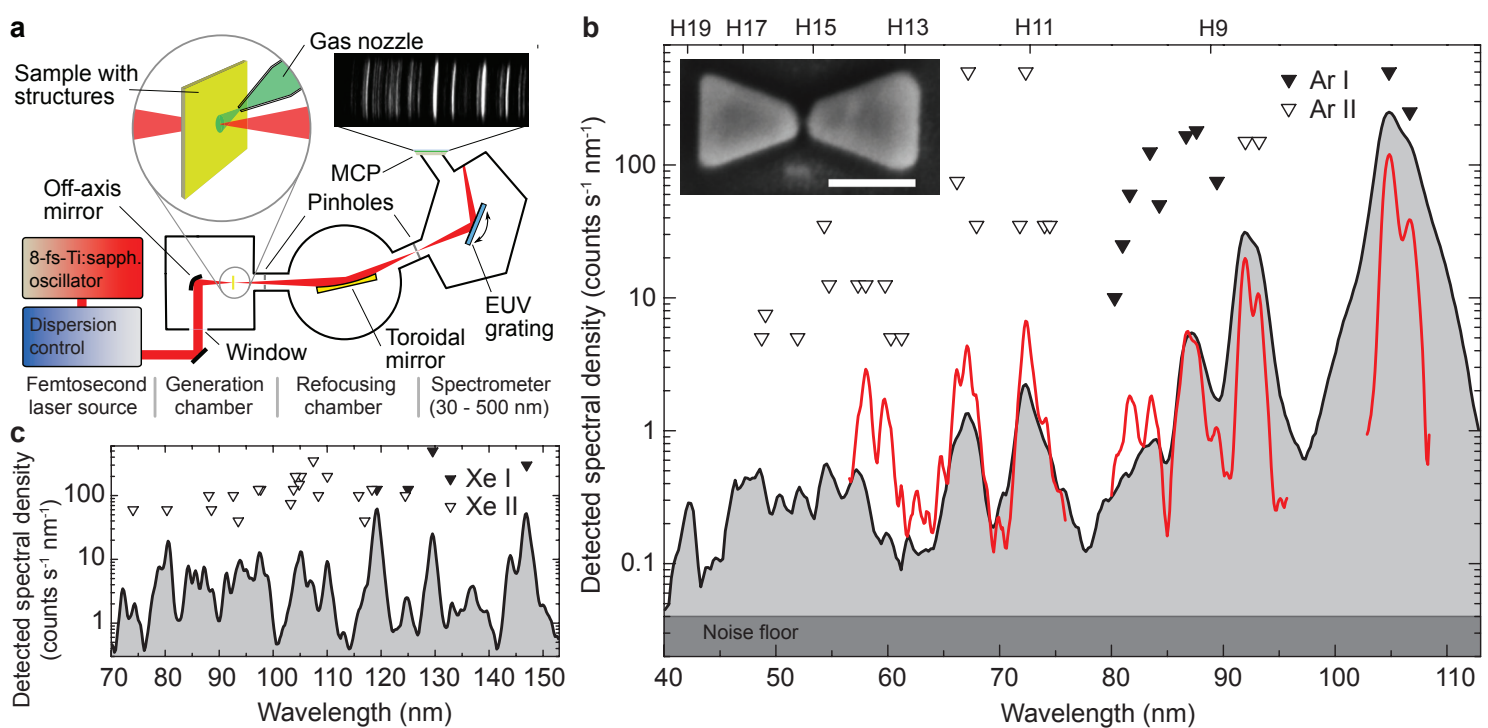

Figure 2.1: Experimental set-up and results. a, Diagram of the experimental set-up. Inset, image of the phosphor screen recorded with a CCD camera. MCP, microchannel plate. This image corresponds to the xenon measurement shown in c. b. Detected spectral density (solid black line) from an array $\left(20 \times 20 \mu \mathrm{m}^{2}\right)$ of argon-exposed nanostructures (inset; scale bar, $200 \mathrm{~nm}$ ) illuminated by femtosecond laser pulses. The second grating diffraction order (solid red line) provides higher resolution and efficiency at shorter wavelengths, and it is shown wherever it does not overlap with other orders. The emission corresponds to atomic line emission from neutral (Ar I; filled triangles) and singly ionized (Ar II; open triangles) argon. Vertical triangle positions indicate expected relative intensities $[54,55,56]$. Note the wavelengths expected for HHG using $800 \mathrm{~nm}$ light (H9-H19, upper $x$-axis). c, Spectrum measured using xenon and the same nanostructure as in $b$ (first grating diffraction order). Triangles indicate the expected xenon lines [56, 57]. Filled triangles are upshifted by a factor of 10 for better visibility.

intensities beyond damage thresholds. This is a striking result, considering that the small detection solid angle in the set-up strongly favours directional emission (HHG) over incoherent line emission. There are fundamental physical reasons for the predominance of line emission in this geometry, as we discuss below. In ref. [24], using ordinary gas densities, the authors claim conversion efficiencies similar to conventional HHG. However, the much smaller number of coherently emitting dipoles, entering quadratically in the yield, suggests a huge deficit in the conversion efficiency of nanostructure-enhanced HHG. A simplified expression for the ratio of expected conversion efficiencies for nanostructureenhanced $\left(C_{\text {nano }}\right)$ and conventional $\left(C_{\text {conv }}\right)$ HHG (using amplified pulses in a capillary or 
gas jet) at comparable local intensities is given by

$$
\frac{C_{\text {nano }}}{C_{\text {conv }}}=\frac{R_{\text {nano }}}{R_{\text {conv }}}\left(\frac{N_{\text {nano }}}{N_{\text {conv }}}\right)^{2} \frac{\left|F_{\text {nano }}\right|^{2}}{\left|F_{\text {conv }}\right|^{2}} \lesssim 10^{-8}
$$

where $N_{\text {nano }} / N_{\text {conv }} \approx 10^{-8}$ and $R_{\text {nano }} / R_{\text {conv }} \approx 10^{5}$ are the ratios of the number of radiating atoms (at comparable density) and repetition rates in both scenarios, respectively. A typical phase matching coefficient $\left|F_{\text {conv }}\right|^{2} \gtrsim 10^{-3}$ is assumed for the relevant harmonics [19], while nanostructure-based HHG is assigned $\left|F_{\text {nano }}\right|^{2}=1$. Such considerations may also be relevant for related studies [26]. Note that, because of a linear dependence on the dipole number for incoherent radiation, the above unfavourable conversion efficiency does not apply to atomic line emission. Thus, it is efficiently enhanced in nanostructures, as demonstrated here. In fact, a generation rate of incoherent fluorescence photons greater than $10^{9} \mathrm{~s}^{-1}$ is estimated from our raw data and collection conditions.

Despite experiments with numerous high-quality samples of different dimensions (displaying efficient third harmonic generation), optimizations of gas nozzle dimensions, materials and orientations, as well as gas pressures, we have only observed atomic and ionic line emission and were able to reproduce our findings multiple times. Thus, together with the physical arguments given above, we must conclude that very efficient HHG in bow-tie nanostructures under the given conditions is highly unlikely, if not physically impossible.

We believe that our results are difficult to reconcile with the conclusions of Kim et al. [24], and further note several of our observations that are at variance with their results. First, in our experiments, we always observe second and higher grating diffraction orders, which is expected for broadband EUV gratings such as the ones used here and in ref. [24], where higher diffraction orders are absent. Second, the photon count rates in our experiments did not exceed several thousand per second using an imaging detector and obtaining a signal to noise ratio of $\sim 10^{3}$. In contrast, ref. [24] reports photon count rates above $10^{8} \mathrm{~s}^{-1}$, even exceeding the laser repetition rate, using a photon multiplier but displaying a signal to noise ratio of only $\sim 10^{2}$. It is very important to distinguish between actual count rates and projected generation rates, which arise from normalization by the quantum efficiency of the set-up; ref. [24] does not state which of these quantities is plotted. Generally, we believe that using conventional photon counting techniques, the nanostructure-enhanced atomic line emission we have found will be detectable in such experiments. Last, the linewidths we have measured are partially given by spectrometer 
resolution (below $2 \mathrm{~nm}$ ) and are very similar to those in ref. [24] and in a related experiment with xenon [58]. Whereas harmonic linewidths are influenced by the spectral amplitude and temporal duration of the fundamental driving field, the linewidths of atomic and ionic fluorescence are governed by the spontaneous lifetime. If harmonic radiation were present, we would expect several linewidths to be broader, given the incident pulse duration and known properties of plasmonic resonances [45, 51, 6].

In conclusion, the line emission observed in our experiments originates from nanostructure-enhanced multiphoton and strong-field excitation and ionization, and is intrinsically incoherent. Moreover, the fundamental physical relations discussed above imply important limitations on nanostructure-enhanced HHG, which calls for alternative approaches.

\section{Methods}

Nanostructures. Numerous arrays (area $20 \times 20 \mu \mathrm{m}^{2}$ ) of bow-ties are fabricated by focused ion-beam etching of smooth gold films (thermal evaporation; $<1 \mathrm{~nm}$ r.m.s. roughness over $5 \times 5 \mu \mathrm{m}^{2}$ ) on EPI polished sapphire substrates. High optical (structural) nanostructure quality is confirmed using optical third harmonic generation (scanning electron/atomic force microscopy). Structural parameters are iteratively optimized for maximum emission (EUV/third harmonic), starting from nominal parameters in ref. [24]. Improved nonlinear emission is found for film thicknesses, bow-tie lengths (single triangle) and gap sizes of $90 \mathrm{~nm}, 230 \mathrm{~nm}$ and $20 \mathrm{~nm}$, respectively. For different arrays, the EUV yield depends on the field enhancement and resonance wavelength.

Experimental set-up (Fig. 2.1a). Optical excitation is provided by focusing dispersioncompensated 8-fs pulses from a Ti:sapphire oscillator with an off-axis parabolic mirror to incident peak intensities of $0.1-1 \mathrm{TW} \mathrm{cm}^{-2}$. Micro-translation stages carry the samples (room temperature); a movable nozzle (stainless steel, inner diameter $100 \mu \mathrm{m}$ ) supplies a gas jet (up to 500 mbar backing pressure). The generated EUV radiation within an opening angle of $\pm 1.2^{\circ}$ is refocused (using a toroidal gold mirror) into a flat-field EUV spectrometer (McPherson 234, 1,200 grooves per mm). Proper alignment of the set-up for collecting possible directed radiation is ensured using the fundamental beam (zeroth grating order) and the third harmonics $(267 \mathrm{~nm})$ from the bare nanostructures and the substrate. EUV emission is detected with a phosphor-screen microchannel-plate assembly (Hamamatsu, uncoated). Accurate wavelength calibration is verified with plasma line emission and conventional HHG using the same set-up and nozzle. 


\title{
Chapter 3
}

\section{Extreme-ultraviolet light generation in plasmonic nanostructures}

\author{
M. Sivis, M. Duwe, B. Abel, and C. Ropers \\ Nature Physics 9, 308 (2013) \\ DOI: $10.1038 /$ nphys 2590
}

Strong-field phenomena in optical nanostructures have enabled the integration of nanophotonics, plasmonics and attosecond spectroscopy. For example, tremendous excitement was sparked by reports of nanostructure-enhanced high-harmonic generation. However, there is growing tension between the great promise held by extreme-ultraviolet and attosecond-pulse generation on the nanoscale, and the lack of successful implementations. Here, we address this problem in a study of highly nonlinear optical processes in gas-exposed bow-tie nanoantennas. We find multiphoton- and strong-field-induced atomic excitation and ionization resulting in extremeultraviolet fluorescence, as well as third- and fifth-harmonic generation intrinsic to the nanostructures. Identifying the intensity-dependent spectral fingerprint of atomic fluorescence, we gauge local plasmonic fields. Whereas intensities sufficient for high-harmonic generation are indeed achieved in the near-field, the nanoscopic volume is found to prohibit an efficient conversion. Our results illustrate opportunities and challenges in highly nonlinear plasmonics and its extension to the extreme ultraviolet.

Tailoring and controlling optical near-fields in plasmonic nanostructures facilitate the enhancement of numerous linear and nonlinear optical effects [45, 50, 51, 52, 53]. Applications are diverse and include surface enhanced Raman scattering [1, 2], second [3, 4] 
and third [5, 6] harmonic generation, two-photon photoluminescence $[59,60]$, continuum generation [61] or localized multiphoton photoemission [7, 8]. Recently, even higher nonlinear effects such as above-threshold and strong-field photoemission and acceleration were observed in plasmonic and other nanostructures [12, 62, 63, 17, 15, 64], closing the gap to strong-field and attosecond physics. [13, 14]

Another key example of this development was the report of high-harmonic generation (HHG) in plasmonic bow-tie antennas [24], that is, the integration of the most prominent strong-field effect of gaseous media [20,33] into a nanostructure. Despite triggering widespread theoretical $[46,65,66,67]$ and experimental $[26,28]$ efforts, subsequently, this effect has remained rather elusive. Important issues in this respect are whether plasmonic nanostructures can generally sustain sufficient intensities for HHG (refs [29, 68]), if other fundamental limitations to the process exist [28], and what the ultimate prospects of ultrafast extreme-ultraviolet (EUV) nano-optics are.

Generally, efficient high-harmonic conversion requires both sufficient intensities and a coherent radiation build-up. Here, we show that whereas the first condition can be realized in nanostructures, the second condition faces serious difficulty compared with conventional approaches [28, 69]. In contrast, incoherent processes such as atomic line emission (ALE), which only require high intensities, are readily enhanced.

To examine the specifics of highly nonlinear, plasmon-enhanced light emission, we carry out experiments with low-energy laser pulses at bow-tie nanoantennas (Fig. 3.1a,b) and compare the emission characteristics with those obtained using high-energy pulses without nanostructures in the same set-up (Fig. 3.1a,c,d), hereafter called conventional scheme.

The nanostructure-enhanced EUV emission in the forward direction is measured (Fig. $3.1 \mathrm{~b})$, whereas the radiation generated by amplified pulses is detected both perpendicular (Fig. 3.1c) and parallel (Fig. 3.1d) to the beam direction, to separate incoherent (ALE) from coherent (HHG) contributions (see Methods for details).

We begin our presentation with the nanostructure-enhanced scenario. Arrays of gold bow-tie structures (Fig. 3.1e) were mounted in a vacuum chamber on micro-translation stages, faced by a moveable gas jet from a stainless-steel nozzle (Fig. 3.1b, inset). High quality structures (focused-ion-beam preparation) of various dimensions (Fig. 3.2d) were illuminated with ultrashort low-energy pulses. Figure 3.2 shows the respective emission spectra recorded using argon (Fig. 3.2a) and xenon (Fig. 3.2b) exposure at incident laser intensities of about $0.15 \mathrm{TW} \mathrm{cm} \mathrm{cm}^{-2}$. Here, the data shown in ref. [28] stem from structure 

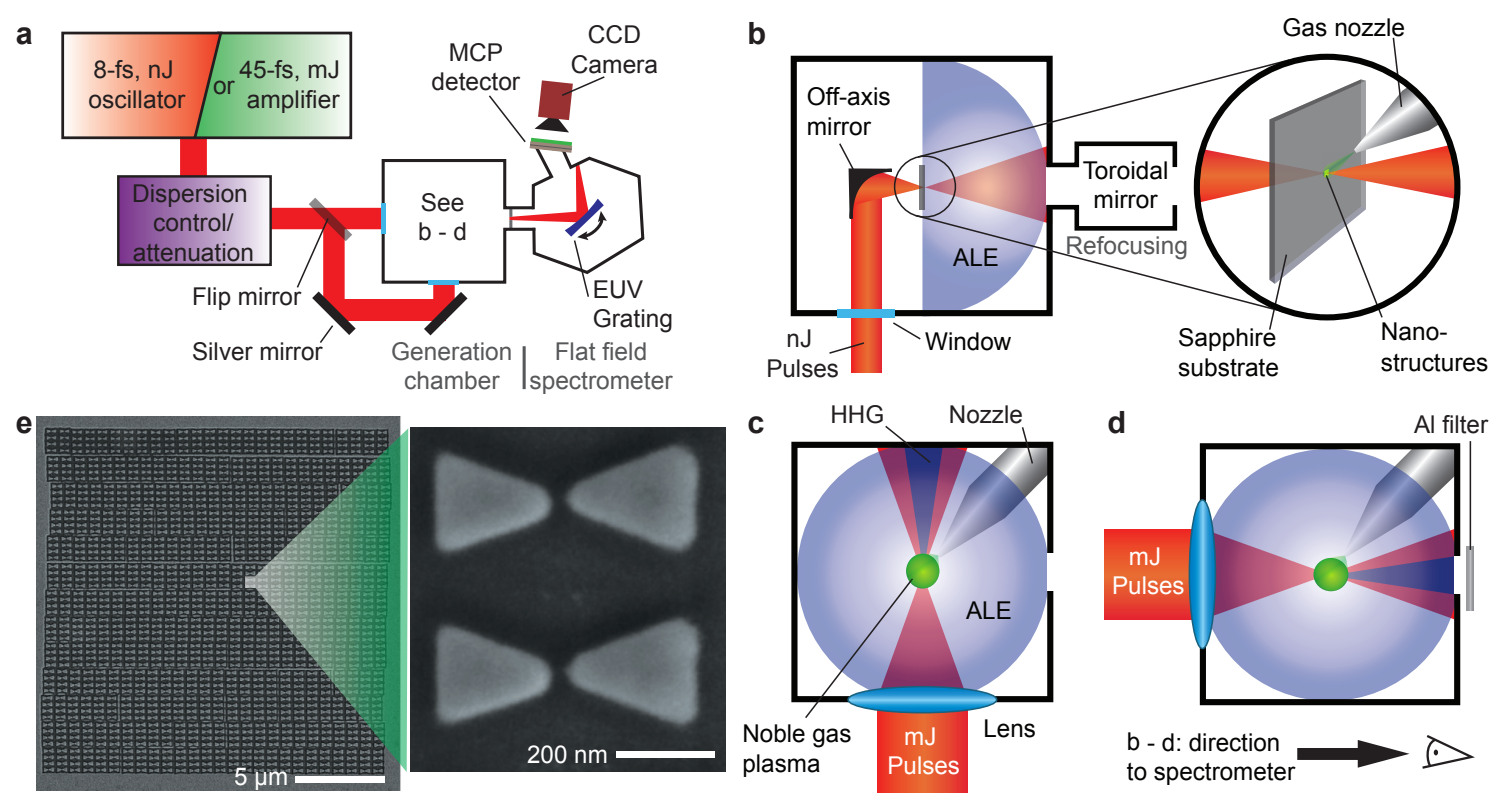

Figure 3.1: Diagrams of the set-up and generation schemes. a, Schematic of the experimental set-up. The top views of the different generation and detection schemes are illustrated in $\mathbf{b}$ d. b, Generation scheme for the nanostructure-enhanced measurements using low-energy laser pulses. c,d, Schematics of the generation chamber for the ALE/HHG reference measurements using amplified pulses and the same gas nozzle as in $\mathbf{b}$. e, Scanning electron micrograph of an array containing bow-tie nanostructures with a close-up view.

iv (solid black). A higher-resolution spectrum measured in the second grating diffraction order is shown in Fig. 3.2c (structure ii). These spectral features are identified as nanostructure-enhanced fluorescence from neutral and ionized gas atoms (that is, ALE), driven by multiphoton excitation and strong-field ionization, and evidenced by a comparison with transitions for neutral (filled triangles) and singly ionized (open triangles) atoms [56]. Some further peaks in the xenon spectra are due to higher ionized states and higher diffraction orders. The different signal levels in these measurements have several causes, including structure-dependent field enhancements and resonance wavelengths.

Despite achieving EUV emission using numerous optimized structures and selectively collecting radiation in a small solid angle in the forward direction (see Methods), we have never found indications for higher-order harmonic generation under these conditions. In contrast, we have observed low-order harmonics intrinsic to the bow-tie nanostructures, most likely stemming from surface harmonic generation in the enhanced fields [70, 71]. In particular, we find the third- and notably the fifth-order harmonics of the driving field (Fig. 3.2e), with a yield that is not significantly influenced by the presence of the gas 
jet. These observations evidence proper alignment, substantial field enhancement and a plasmonic resonance within the incident laser spectrum. The resonance properties of the bow-tie antennas used were characterized by dark-field spectroscopy, as shown in the inset of Fig. 3.2e for the shortest and longest antennas with single arm lengths of $200 \mathrm{~nm}$ and $240 \mathrm{~nm}$, respectively. The resonance wavelengths (between 810 and $940 \mathrm{~nm}$ ) are
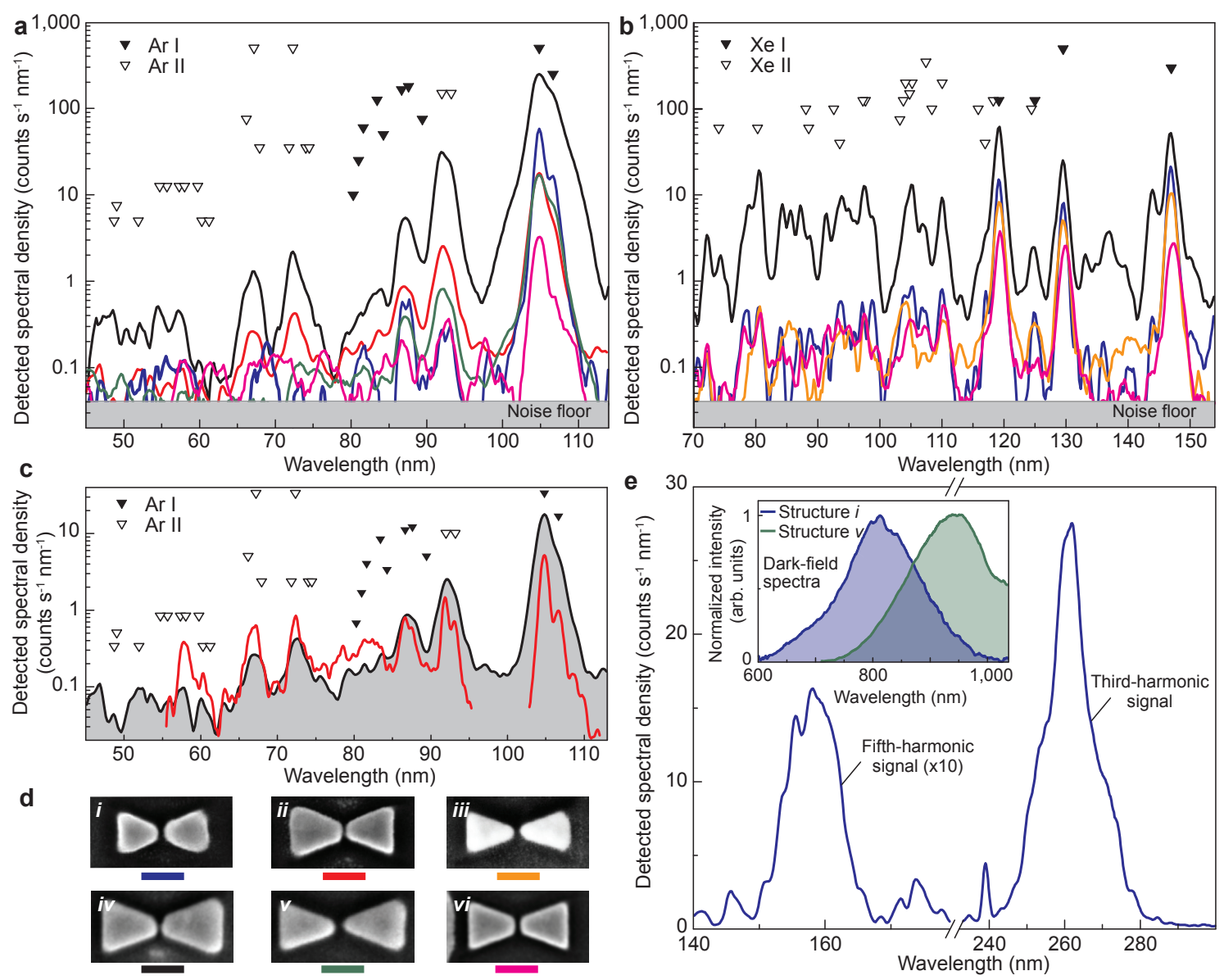

Figure 3.2: Nanostructure-enhanced emission spectra. a,b, EUV spectra using argon (a) and xenon (b) exposure. Line colors refer to bow-tie geometries shown in d. Triangles indicate expected transitions for neutral (filled) and singly ionized (open) gas of the respective element. Vertical triangle positions correspond to reported relative intensities [56]. Xe I triangles are upshifted $(\times 10)$ for better visibility. $\mathbf{c}$, First (black) and second (red) grating diffraction orders of the ALE for argon obtained with the bow-tie geometry displayed in $\mathbf{d}(i i)$. d, Scanning electron micrographs of the different bow-tie antennas. The gold film thickness is $90 \mathrm{~nm}$ and $50 \mathrm{~nm}$ for the structures $i-v$ and $v i$, respectively. Color-coded scale bars, $200 \mathrm{~nm}$. Structure and spectrum $i v$ correspond to those shown in ref. [28]. e, Third- and fifth-harmonic spectra obtained with structure $i$. Inset: dark-field scattering spectra for structure $i$ and $v$. 
consistent with the structures approximately behaving as quarter-wavelength antennas. It should be noted that the maximum field enhancement found is not only a function of antenna length, but also strongly depends on the actual gap size and overall structural quality.

The identification of nanostructure-enhanced ionization and ALE allows for new means to investigate the limits of highly nonlinear physics in optical nanostructures, for example, concerning their durability and maximum achievable local intensities. We have carried out various test measurements to identify conditions for stable operation and the peak intensities, at which accelerated degradation sets in. Bow-tie structures on sapphire substrates (unlike on mica, see Fig. 3.3a) were found capable of sustaining local intensities sufficient for EUV generation for at least a few hours. As an illustration, Fig. 3.3b shows scanning electron micrographs of sample (iv) directly after fabrication, after prolonged EUV generation (over several hours) with argon and xenon at incident intensities up to $0.15 \mathrm{TW} \mathrm{cm}^{-2}$ (including the measurements in Fig. 3.2), and at several minutes of exposure to intensities up to $0.3 \mathrm{TW} \mathrm{cm}^{-2}$. Whereas only rather minor modifications in the gap region can be identified in the second image, the third image shows significant accelerated degradation, especially in the region of largest field enhancement, which coincided with an irreversible loss of signal. This shows that already such subtle sample alterations without complete damage [68] may lead to a loss of field enhancement and EUV generation. During the exploration of the intensity regime beyond the damage threshold, we have not observed any transient occurrence of HHG. Similar tests were carried out for numerous structures, with the same result.

It is important to resolve whether the lack of any HHG signatures in these measurements is caused by insufficient local intensities or has other physical origins. The presence of low-order harmonics and substantial gas ionization already implies rather high intensities within the nanostructures $[72,73,74]$. (Note that the ionization energies of the gases used (argon: $15.8 \mathrm{eV}$, xenon: $12.1 \mathrm{eV}$ ) exceed the energy of ten and seven laser photons, respectively.) However, absolute local intensities are difficult to estimate based on total yields. In general, the total strength of optical signals generated in nanostructures not only depends on the near-field intensity but on multiple additional factors, which may include chemical effects, quenching, quantum efficiencies or specimen density. Therefore, an independent measure of local intensities is desirable, and we have found that reference measurements using amplified femtosecond pulses can provide an absolute intensity scale. 
Figure 3.4a contains ALE spectra from amplified pulses obtained in the side-view collection geometry of Fig. 3.1c. For comparison, one of the nanostructure-enhanced ALE spectra is superimposed (thick gray line). It is apparent that both spectra contain the same features, although a lower spectral resolution (caused by the refocusing optics) was achieved in the nanostructure case. Furthermore, it can be seen that the relative strengths of some of the emission lines are changing with intensity, which is clearly visible for the lines at $104.8 \mathrm{~nm}$ and $106.7 \mathrm{~nm}$ wavelength.

An intensity-dependent measurement of the fluorescence yield of these two lines (Fig. 3.4d) uncovers the origin of this effect. The yields of the $104.8 \mathrm{~nm}$ and $106.7 \mathrm{~nm}$ lines show slopes of 8 and 10 in a double-logarithmic plot, respectively, before approaching a slope of unity. The excitation of the $104.8 \mathrm{~nm}$ line is most likely caused by direct eightphoton absorption and subsequent radiative decay to the ground state. In contrast, the $106.7 \mathrm{~nm}$ line has a smaller transition dipole moment for direct excitation [75], and the slope of 10 suggests an indirect excitation path via higher-lying states, which will be a subject of further study.

This intensity-dependent spectral ALE fingerprint may serve as a direct probe for gauging high local intensities in nanostructures by evaluating the relative line strengths in a
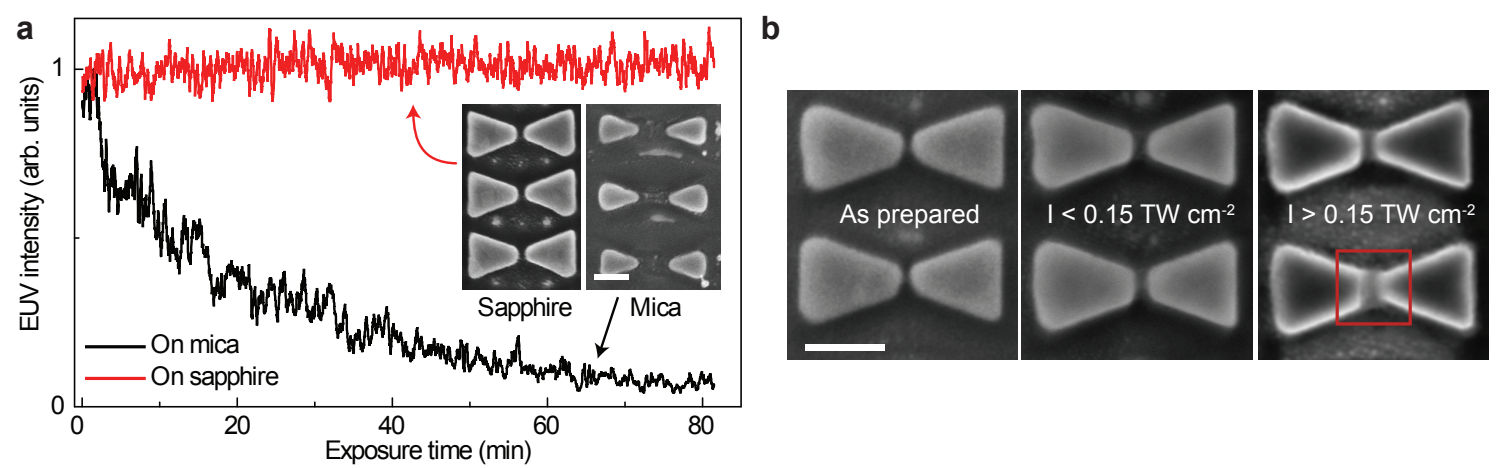

Figure 3.3: Durability measurements. a, Long-term EUV yield measurement of bow-tie nanostructures on mica (black) and sapphire (red) substrates. Nanostructures on sapphire substrates are found to remain intact for substantially longer times than nominally identical structures on mica substrates, which display cumulative damage effects (see right inset). The insets show scanning electron micrographs recorded after the respective experiments. b. Scanning electron micrographs of structure $i v$ directly after preparation through focused-ion-beam etching, after several hours of exposure to incident intensities up to $0.15 \mathrm{TW} \mathrm{cm}{ }^{-2}$ and few minutes at higher intensities up to $0.3 \mathrm{TW} \mathrm{cm}^{-2}$. The melting of the structure in the gap region is clearly visible (red square). Scale bars, $200 \mathrm{~nm}$. 
similar manner. This approach is related to the concept presented in ref. [76] and allows for sensing in much higher fields and smaller interaction volumes. Figure 3.4c shows the intensity-dependent ratio of line strengths $\mathrm{I}_{106.7} / \mathrm{I}_{104.8}$ for the (amplifier) reference measurements and a measurement using nanostructures (here: structure $i v$ ). Both measurements show an increase of this ratio with incident intensity. Here, the lower (upper)
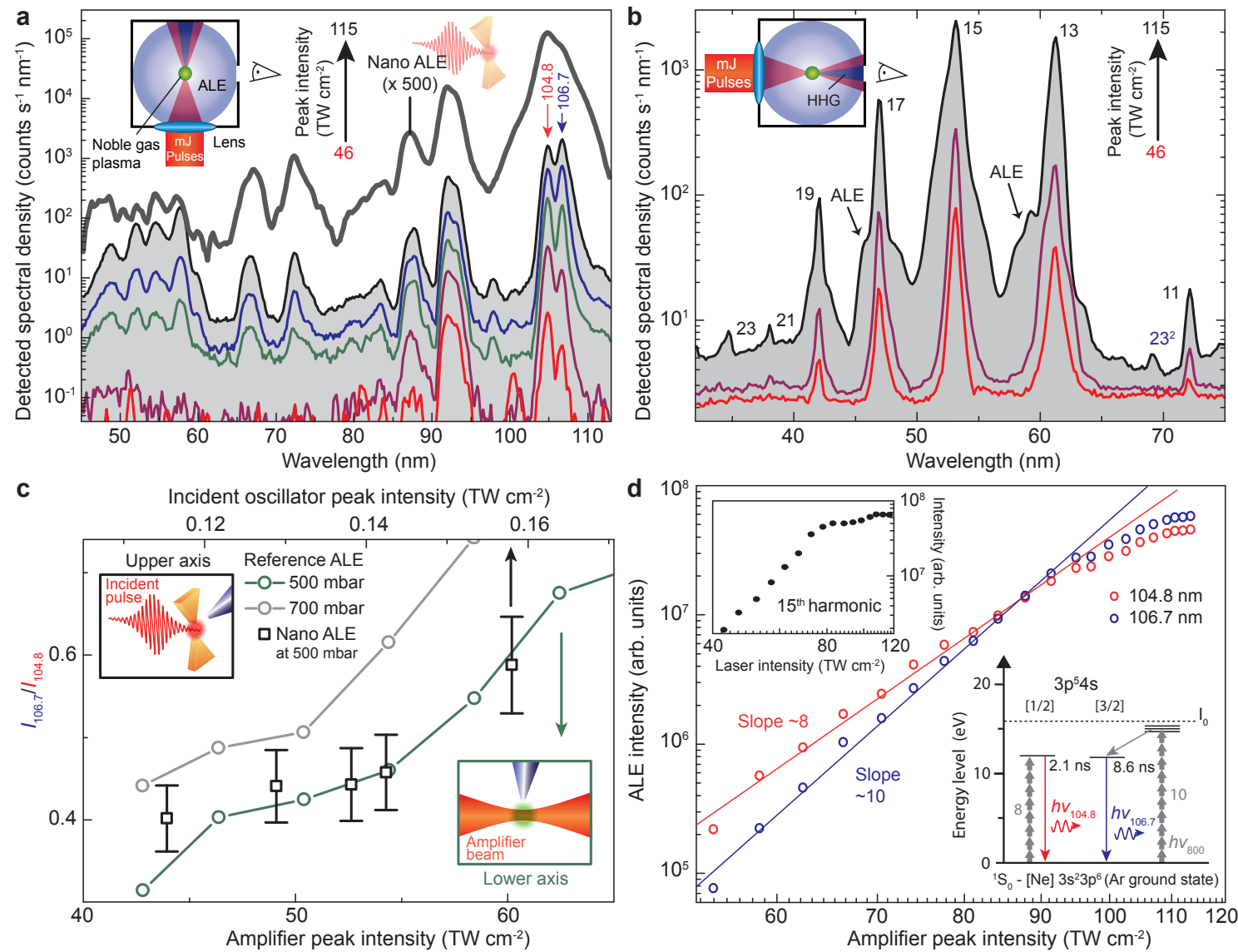

Figure 3.4: Intensity-dependent reference measurements. a, ALE spectra from argon gas (first diffraction order) for different incident laser peak intensities using amplified pulses (red to black), detected in side-view geometry. b, HHG spectra detected along the beam direction. Peaks correspond to the odd harmonic orders (labeled from 11th to 23th) of the pump (central wavelength: $800 \mathrm{~nm}$ ). An Al filter suppresses wavelengths greater than $80 \mathrm{~nm}$. Additional side peaks are identified as ALE. c, Peak-intensity-dependent ratio of the argon line strengths at $104.8 \mathrm{~nm}$ and $106.7 \mathrm{~nm}$ wavelength for the reference (lower axis) and the nanostructureenhanced (upper axis) measurements. The error bars were extracted from temporal signal fluctuations caused by, for example, local gas pressure variations. d, Peak-intensity-dependent signal strengths of the 104.8 and $106.7 \mathrm{~nm}$ lines. Insets: intensity-dependent signal of the 15 th harmonic in b. Diagram of the relevant energy levels for neutral argon. 
axis refers to the focus peak intensities of the measurements without (with) nanostructure enhancement. Thus, by comparing these measurements and the incident intensities required to obtain a particular line strength ratio, we can estimate the intensity enhancement provided by the nanostructures and the local intensities achieved in a given experiment. At present, this also assumes a weak pulse duration dependence of the spectral fingerprint. In Fig. 3.4c, best agreement with the amplifier reference measurements is obtained for a nanostructure intensity enhancement of 380, which agrees with theoretically predicted values for bow-ties [46] and suggests local intensities up to $60 \mathrm{TW} \mathrm{cm}^{-2}$ within the nanostructures. An uncertainty of about $15 \%$ arises from the pressure dependence of these signals (see grey line in Fig. 3.4c).

Thus, intensities within the nanostructures are clearly beyond the HHG threshold, as evident from expected cutoffs [20] and experimentally seen in Fig. 3.4b (red line: $46 \mathrm{TW} \mathrm{cm}^{-2}$ ). Here, high-harmonic radiation from the amplifier pulses is collected at some of the intensities used in Fig. 3.4a. Such measurements directly illustrate that although the intensities in nanostructures may indeed be sufficient to achieve ionization conditions equalling those using a conventional scheme, the resulting emission in the forward direction is still of a very different nature. In particular, whereas the forward emission in the conventional case is dominated by the coherent HHG contribution (Fig. 3.4b), the nanostructure-enhanced scenario shows no sign of this emission.

From the above results, we conclude that it is not a lack of local intensity that causes the absence of high-harmonic radiation in our nanostructure EUV spectra. Instead, the coherent build-up within the small nanostructure propagation length seems to be insufficient to raise the intensity of coherent emission beyond the level of incoherent ALE.

Previously, we have made a comparison of the conversion efficiencies in conventional and nanostructure-enhanced HHG (ref. [28]). However, such an estimate only illustrates the general difficulty in achieving coherent signals in nanometric volumes, as the conversion efficiency in a given implementation critically depends on numerous experimental factors, including collection angles, interaction lengths or beam parameters. Here, we discuss expected relative yields by directly using our own reference data taken in the same set-up (Fig. 3.4). The reference measurements were conducted such that the interaction length was shorter than the coherence lengths arising from both plasma dispersion and geometrical dephasing [33] (see Methods). Although such conditions are not optimized for maximum conversion efficiency, they allow us to assume a quadratic scaling of the HHG signal with interaction length. We can thus carry out a rough estimation of the 
expected strength of a potential nanostructure-enhanced HHG signal $S_{H H G \text {,nano }}$ relative to the observed ALE emission $S_{A L E, \text { nano }}$, using the measured ratio $S_{H H G, r e f} / S_{A L E, \text { ref }}$ in the reference measurements:

$$
\frac{S_{H H G, \text { nano }}}{S_{A L E, \text { nano }}} \approx \frac{S_{H H G, \text { ref }}}{S_{A L E, \text { ref }}} \frac{L_{\text {nano }}}{L_{\text {ref }}}
$$

Here, the interaction lengths $L_{r e f}$ and $L_{\text {nano }}$ are about $300 \mu \mathrm{m}$ and $100 \mathrm{~nm}$, respectively. Empirically, the ratio $S_{H H G, r e f} / S_{A L E \text {,ref }}$ near a wavelength of $60 \mathrm{~nm}$ was intensity dependent and varied between 14 and 20 above $50 \mathrm{TW} \mathrm{cm}^{-2}$, where $S_{A L E \text {,ref }}$ was detectable (Fig. 3.4b). The relative contribution of the coherent HHG radiation to the overall EUV signal is therefore reduced by $L_{\text {nano }} / L_{r e f} \approx 3 \times 10^{-4}$, so that we may expect a nanostructure-enhanced HHG intensity that is lower than that of the ALE by a factor of about $6 \times 10^{-3}$ (taking the maximum value of $S_{H H G, r e f} / S_{A L E, r e f} \approx 20$ ). Although such signals are potentially measurable, simple relations such as equation 3.1 illustrate that it will be very challenging to detect any clean HHG spectrum from nanostructures that does not have a significant or even dominant incoherent contribution.

In addition, diffraction caused by the geometrical arrangement of the nanostructure array and the subwavelength nature of the bow-tie gap further diminishes any coherent radiation that may be collected in the forward direction. An estimate of the relative power in the zeroth diffraction order of the structure is obtained by viewing the bow tie gaps as coherent (scalar) emitters with a diameter of the gap size $g$, spaced by the distances $D_{x}$ and $D_{y}$ in a planar rectangular array, and emitting with an overall envelope on the order of the laser focus diameter. For a more complete consideration, also the source extension in the propagation direction would have to be included, at least for cases where the wavelength is comparable or shorter than this extension. At the EUV wavelength $\lambda$, about $n=\left(D_{x} D_{y}\right) / \lambda^{2}$ diffraction orders exist in the far-field. In these experiments, $g$ is significantly smaller than $\lambda$. Therefore, one may assume that all diffraction orders in the far-field would be about equally populated in intensity, and a relative fraction on the order of only $1 / n$ of the total emitted power would be emitted in the forward direction (zeroth order). For our structures, this would correspond to at most few per cent of the total emitted power, which further reduces the likelihood of observing HHG from nanostructures. Overall, we believe that both the minute interaction length and the significant influence of diffraction from subwavelength generation volumes have important ramifications for the practicality of nanostructure-enhanced HHG.

Our investigation of highly nonlinear processes and local intensity probing in plasmonic 
nanostructures demonstrates the feasibility of studying various strong-field phenomena in optical near-fields, such as EUV generation, but also illustrates severe constraints for coherent radiation build-up. We believe that for future implementations of nanostructurebased EUV generation, it is very important to carefully and spectroscopically identify relative contributions from incoherent and coherent processes. To become competitive with other HHG concepts at high repetition rates [77, 78, 79], the number of participating atomic dipoles should be substantially increased using higher pressures and larger interaction volumes. Nonetheless, the observation of stable nonlinear fluorescence signals is encouraging and may find application where spatial and temporal coherence are not essential, for example in an implementation of near-field EUV lithography. Finally, the demonstrated approach to use the spectral fingerprint of atomic gases can be further refined to ultimately serve as a quantitative local gauge for plasmonic fields and atomic densities. Alongside the observation of plasmonic fifth-harmonic generation, this opens up the study of radiation and optical coupling effects within metallic nanostructures at ultraviolet and EUV frequencies, a widely unexplored field. 


\section{Methods}

Nanostructure fabrication. The nanostructures used in these experiments are arrays of bow-tie antennas (Fig. 3.1e) prepared by focused-ion-beam etching of thermally evaporated gold films (50-90 nm thickness; $<1 \mathrm{~nm}$ r.m.s. roughness over $5 \times 5 \mu \mathrm{m}^{2}$ area) on dielectric substrates (sapphire or mica). Samples with triangle lengths between $200 \mathrm{~nm}$ and $240 \mathrm{~nm}$ were prepared. Both the radii of curvature and the distance of the opposing bow-tie elements are about $20 \mathrm{~nm}$. (Fig. 3.2d)

Nanostructure experiments. Dispersion-controlled optical excitation is provided at normal incidence by a $78 \mathrm{MHz}$ titanium:sapphire laser oscillator emitting 8-fs light pulses with a centre wavelength of $800 \mathrm{~nm}$ (Femtolasers Rainbow), polarized along the bow-tie main axis, focused to a spot size of $15 \mu \mathrm{m}$ by a parabolic off-axis mirror. A toroidal grazing-incidence gold mirror collects the radiation emitted from the structures in a small solid angle of $\pm 1.2^{\circ}$ and focuses it on the entrance slit of an EUV flat-field spectrometer. Spectra are recorded by a phosphor screen microchannel plate and a CCD (charge-coupled device) camera. The stainless-steel gas nozzle had an aperture diameter of $100 \mu \mathrm{m}$ and is operated at backing pressures of typically 500 mbar.

Reference experiments. Figure 3.1c,d shows two configurations of the generation chamber for the reference measurements, using the same gas nozzle and spectrometer as with nanostructures. ALE and HHG (Fig. 3.1c,d) are observed by direct excitation of the gas jet with amplified laser pulses (800 nm wavelength, 45-fs pulse duration, $0.5 \mathrm{~mJ}$ pulse energy, $1 \mathrm{kHz}$ repetition rate). In the HHG measurements, the fundamental laser beam was blocked by a 150 -nm-thick aluminum filter. The pressure and jet conditions (including focus distance from nozzle exit) were chosen to match those in the nanostructure experiments as closely as possible. The resulting interaction length of the laser field with the gas (about $300 \mu \mathrm{m}$ ) is smaller than the coherence lengths for both geometrical $L_{g, f s}=\pi z_{0} / N \approx 840 \mu \mathrm{m}$ and plasma $L_{f e}=2 \pi c \omega_{0} / \omega_{p}(\tau)^{2} N \approx 2.1 \mathrm{~mm}$ dephasing [33]. In the calculation of the coherence lengths the following experimental parameters are used: Rayleigh length $z_{0}=4 \mathrm{~mm}$, harmonic order $\mathrm{N}=15$, atomic density $n_{a}=1.25 \times 10^{19} \mathrm{~cm}^{-3}$ (500 mbar), ionization fraction $n_{e}(\tau) / n_{a}=3.5 \times 10^{-3}$ (for $60 \mathrm{TW} \mathrm{cm}{ }^{-2}$ intensity in argon [80]) and plasma frequency $\omega_{p}(\tau)^{2}=1.39 \times 10^{-4} \mathrm{fs}^{-2}$. Although the pulse duration in the reference measurements was significantly longer than 
in the nanostructure case, it may be assumed that this does not significantly alter the estimated relative yields because the present conditions are at rather low ionization fractions. Moreover, considering the small interaction length in the gas jet, there would be no qualitative change in using even shorter pulses resulting in longer coherence lengths for a given peak intensity in the reference measurements.

\section{Acknowledgments}

We thank Y. Liu and K. R. Sieferman for their helpful participation in preparatory studies, D. R. Solli and G. Herink for fruitful discussions, P. Simon, E. Lugovoy and V. Radisch for technical support and equipment, and Venteon Femtosecond Laser Technologies for providing a laser oscillator for initial experiments. Financial support by the Deutsche Forschungsgemeinschaft (DFG-ZUK 45/1 and SFB 755) is gratefully acknowledged.

\section{Author contribution}

M.S., B.A. and C.R. conceived and designed the experiment. M.S. and M.D. prepared the nanostructures and conducted the experiments. M.S., M.D. and C.R. analyzed the measured data. M.S. and C.R. wrote the manuscript, with contributions from M.D. and B.A.. All authors were involved in intensive discussions at all stages of the study. 


\title{
Chapter 4
}

\section{Generation and Bistability of a Waveguide Nanoplasma Observed by Enhanced Extreme-Ultraviolet Fluorescence}

\author{
M. Sivis, and C. Ropers \\ Physical Review Letters 111, 085001 (2013) \\ DOI: 10.1103/PhysRevLett.111.085001
}

\begin{abstract}
We present a study of the highly nonlinear optical excitation of noble gases in tapered hollow waveguides using few-femtosecond laser pulses. The local plasmonic field enhancement induces the generation of a nanometric plasma, resulting in incoherent extreme-ultraviolet fluorescence from optical transitions of neutral and ionized xenon, argon, and neon. Despite sufficient intensity in the waveguide, high-order harmonic generation is not observed. The fluorescent emission exhibits a strong bistability manifest as an intensity hysteresis, giving strong indications for multistep collisional excitations.
\end{abstract}

Recent years have shown significant progress in translating concepts from attosecond and strong-field science [22] into the areas of ultrafast nano-optics and plasmonics. Numerous characteristic nonlinear optical phenomena, such as above-threshold and strongfield photoemission, have become accessible using local field enhancements in metal nano- tips [12, 62, 13, 14, 15], resonant optical antennas [24, 28, 16, 81], nanoparticles [17], rough surfaces [63], and plasmonic waveguides [26, 15]. These experimental studies are paralleled by increasing theoretical efforts of describing strong-field effects at surfaces and in optical near fields [82, 83, 64], with a particular emphasis on 
nanostructure-enhanced high-order harmonic generation (HHG) $[46,65,66,84,67,85$, $86,87,88,89,90]$. Currently, some debate exists about experimental reports of nanostructure-enhanced HHG [24, 26, 15], following the demonstration of the predominance of incoherent extreme-ultraviolet (EUV) fluorescence caused by multiphoton and strong-field atomic excitation and ionization [28, 81]. The essential physical issue revolves around the tendency of gaseous media in nanoscopic generation volumes to favor incoherent over coherent processes. Thus, although not all expectations on realizing novel forms of attosecond spectroscopy in compact devices may ultimately materialize experimentally, the discussion reopens the field and further highlights the specificities and alternative scalings exhibited by strong-field interactions in nanostructures. This will allow for entirely new perspectives and phenomena in near-field high-intensity atomic and molecular gas excitations.

In this Letter, we demonstrate the nanostructure-enhanced formation of a confined plasma within a gas-filled plasmonic waveguide using low-energy, few-femtosecond laser pulses. This nanoplasma exhibits a pronounced threshold behavior and bistability of the fluorescent EUV emission that depends on the gas species and pressure. Specifically, we investigate the optical excitation, ionization, and emission characteristics of xenon, argon and neon atoms and ions, finding a strong intensity hysteresis of the fluorescence signal. Moreover, the exclusive observation of fluorescent emission highlights physical limits of nanostructure-based HHG concepts. Our results illustrate the plasmonic enhancement of strong-field effects in gaseous media and provide insight into the mechanisms associated with such phenomena driven at high repetition rates and in nanoscale confinement.

The setup of our experiment is depicted in Fig. 4.1(a). An electrochemically etched gold tip containing the waveguide structures [cf. Fig. 4.1(b)] is placed in a cell that can be purged with different gases. The structures are mounted on microtranslation stages (three translational and one rotational positioner) in a high vacuum chamber. Optical excitation with variable linear polarization is provided by 8 -fs pulses ( $800 \mathrm{~nm}$ center wavelength) from a $78 \mathrm{MHz}$ Ti:sapphire laser oscillator, focused to a $5 \mu \mathrm{m}$ spot size (FWHM). Incident peak intensities up to about $1 \mathrm{TW} / \mathrm{cm}^{2}$ are achieved. A $200 \mu \mathrm{m}$ aperture in the gas cell allows for differential pumping of the detection chamber and the collection of the generated radiation within a solid angle of $\pm 1.2^{\circ}$. Spectral analysis of the collected emission is carried out with a home-built EUV flat-field spectrometer and a phosphor screen microchannel-plate detector. A more detailed description of the detection scheme is given elsewhere $[28,81]$. 


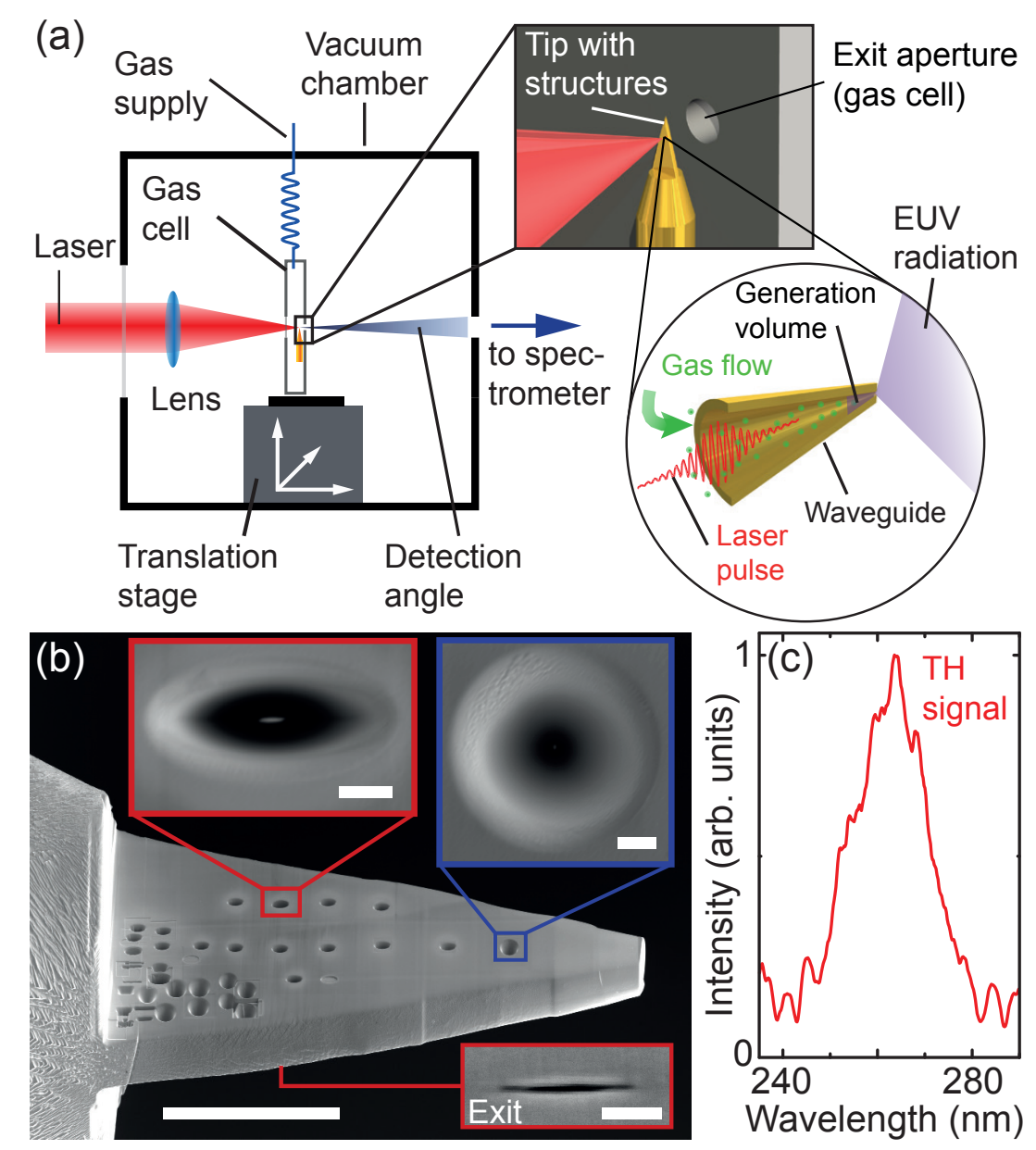

Figure 4.1: (a) Schematics of the experimental setup. The generated radiation in a solid angle of $\pm 1.2^{\circ}$ is spectrally analyzed with a flat-field EUV spectrometer. (b) Scanning electron micrograph of the gold platform containing the tapered waveguides. Upper insets: Close-up views of the entrance apertures of an elliptical and a circular waveguide. Lower inset: Exit aperture of the elliptical waveguide. (Scale bars: overview $50 \mu \mathrm{m}$, top views $1 \mu \mathrm{m}$, exit aperture $200 \mathrm{~nm}$.) (c) Third harmonic signal generated in a waveguide without gas exposure.

Various waveguides with circular and elliptical cross sections are prepared on a $10 \mu \mathrm{m}$ thick gold platform using focused ion beam etching [cf. Fig. 4.1(b)], and we have taken special care to produce smooth waveguide surfaces for minimal losses. For waveguides with elliptical entrance and exit apertures, geometrical dimensions are chosen that approximately correspond to those presented in Ref. [26], in which simulations indicated intensity enhancements of up to 350 near the waveguide end. As an example, Fig. 4.1(b) (top left inset, red) shows a waveguide with diameters of the minor and major axes of the entrance (exit) apertures of $2.4 \mu \mathrm{m}(40 \mathrm{~nm})$ and $4.8 \mu \mathrm{m}(440 \mathrm{~nm})$, respectively. 
Previously, optical second harmonic generation was used to characterize local fields at the exit aperture [91] of hollow near-field probes [92]. Here, we utilize surface-enhanced third harmonic (TH) generation as a confirmation of field enhancement and efficient waveguide coupling [71, 5, 6, 93, 81]. Figure 4.1(c) displays a TH spectrum obtained from an illuminated waveguide in the absence of gas. To ensure a proper alignment and detection of any possible coherent contribution in the EUV radiation, the TH signal was optimized by adjusting the lateral positioning of the structures and the angle of incidence.

In the following, we present our observations of the EUV radiation emitted from the illuminated structures exposed to atomic gases. Figure 4.2(a) shows EUV spectra measured using xenon, argon, and neon gases in the elliptical waveguide displayed in Fig. 4.1(b) (top left inset, red). The spectra exhibit numerous emission lines at the atomic and ionic transitions of the respective elements (indicated by triangles [56]) and thus present incoherent characteristic EUV fluorescence, in other words, atomic line emission. One of the essential features of the hollow waveguide structures, which has not yet been experimentally studied, is the theoretically proposed improvement in the field enhancement of an elliptical over a circular cross section [26] and potential polarization dependencies. We have observed only minor EUV signals from waveguides with circular cross sections, such as the one displayed in Fig. 4.1(b) (top right inset, blue). Moreover, we have studied the dependence of the EUV emission on incident laser polarization [cf. polar diagram in Fig. 4.3(a)]. Maximum EUV intensity was found for a polarization parallel to the minor waveguide axis (corresponding to an angle of $0^{\circ}$ ), which is consistent with an interpretation that the fundamental excitation for polarization parallel to the minor axis reaches deeper into the waveguide and thus creates higher overall field enhancement.

Despite numerous tests, accurate alignments [cf. Fig 4.3(b)], and recording more than 2000 individual EUV spectra using different waveguides, intensities, gases, and pressures, we have not found any indications for high harmonic generation in this spectral range, which appears to contradict the conclusions of previous reports [26, 15]. For better comparison, let us briefly note the differences between our structures and those presented in Ref. [26]. In principle, our use of gold instead of silver as waveguide material may cause higher absorptive losses [94, 47, 39]. However, since the typical propagation lengths of surface plasmon polaritons in gold waveguides are still much larger than the length of the waveguides used [95, 39], we believe that structural quality and durability play a far more significant role than material selection for achieving stable strong-field conditions. In this respect, using a bulk gold support allowing for smooth waveguide walls and high thermal 


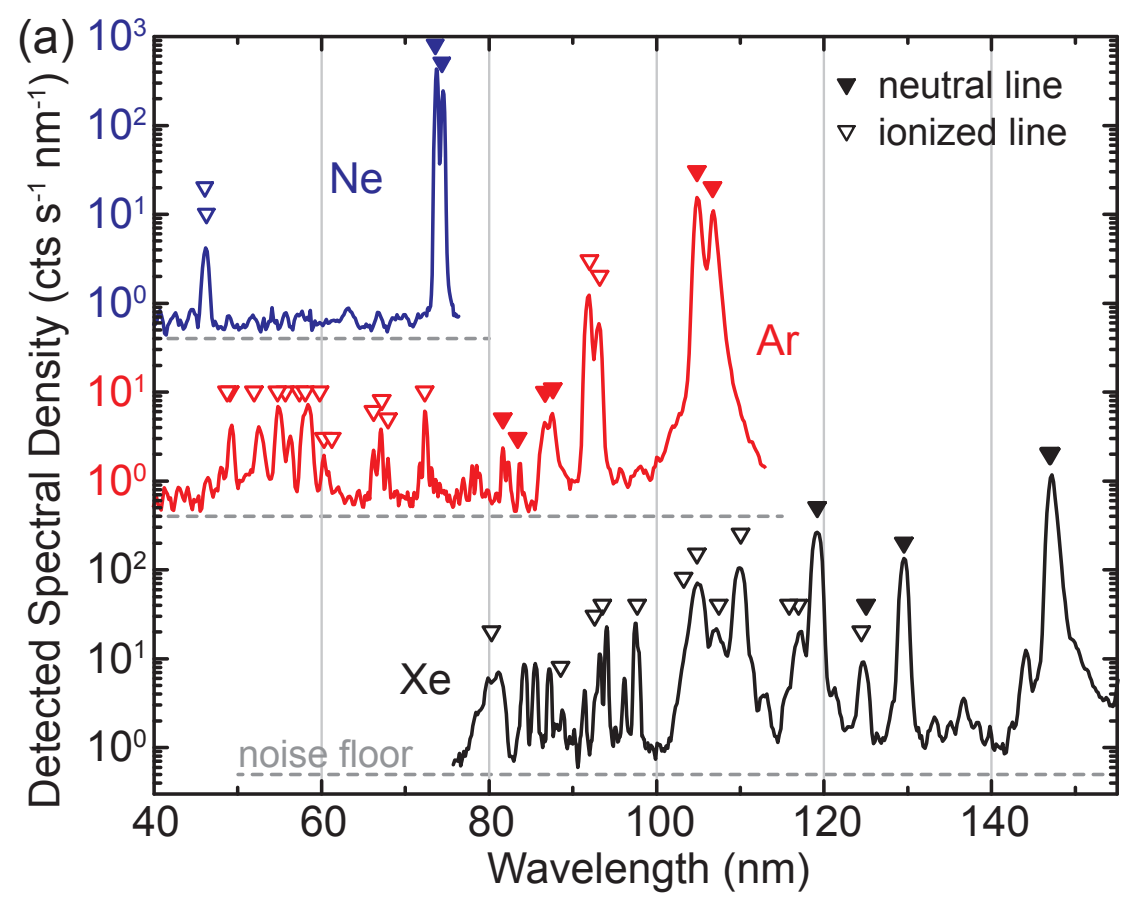

(b)

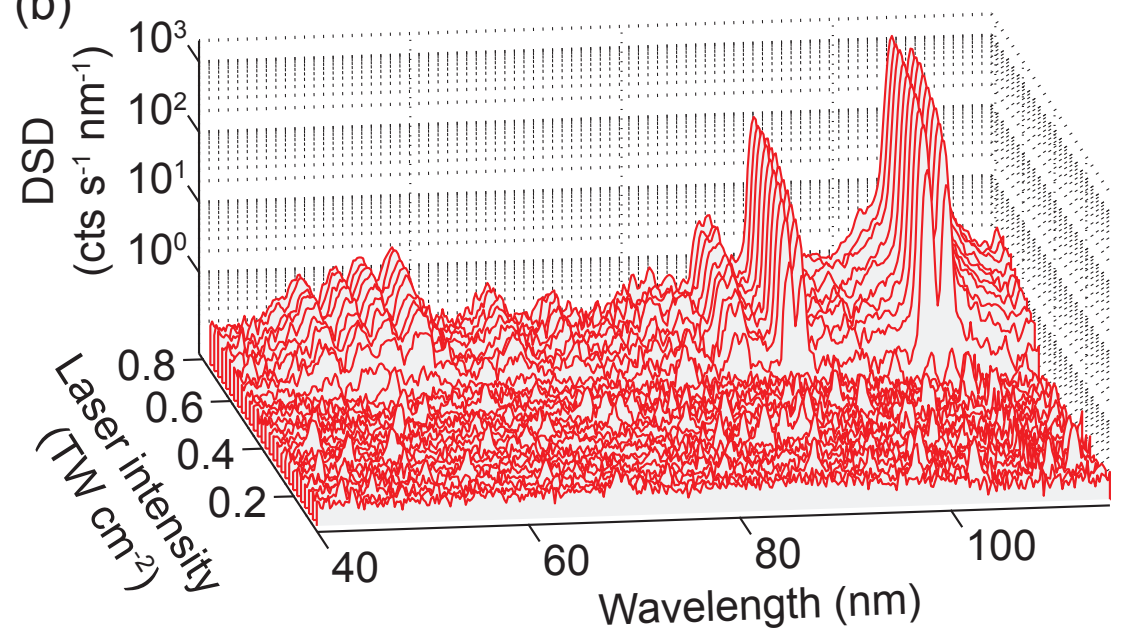

Figure 4.2: (a) Nanostructure-enhanced EUV spectra (logarithmic scale) using xenon, argon and neon gas (200 mbar backing pressure) excited in the waveguide displayed in Fig.4.1(b) (inset, red frame). The argon and neon spectra are up shifted for better visibility. In all cases, dashed gray lines correspond to the same signal level (being the noise floor at 0.5 counts/(s nm) in these measurements). Wavelength positions of triangles indicate the expected fluorescent transitions for neutral (filled) and singly ionized (open) atoms of the respective gas species [56]. Providing for better resolution, the following portions of the spectra were recorded in the second grating diffraction order: $\mathrm{Ne}(>60 \mathrm{~nm})$, Ar $(65-84 \mathrm{~nm})$, Xe $(84-100 \mathrm{~nm})$. (b) Intensity-dependent series of argon spectra recorded for increasing incident laser power. 

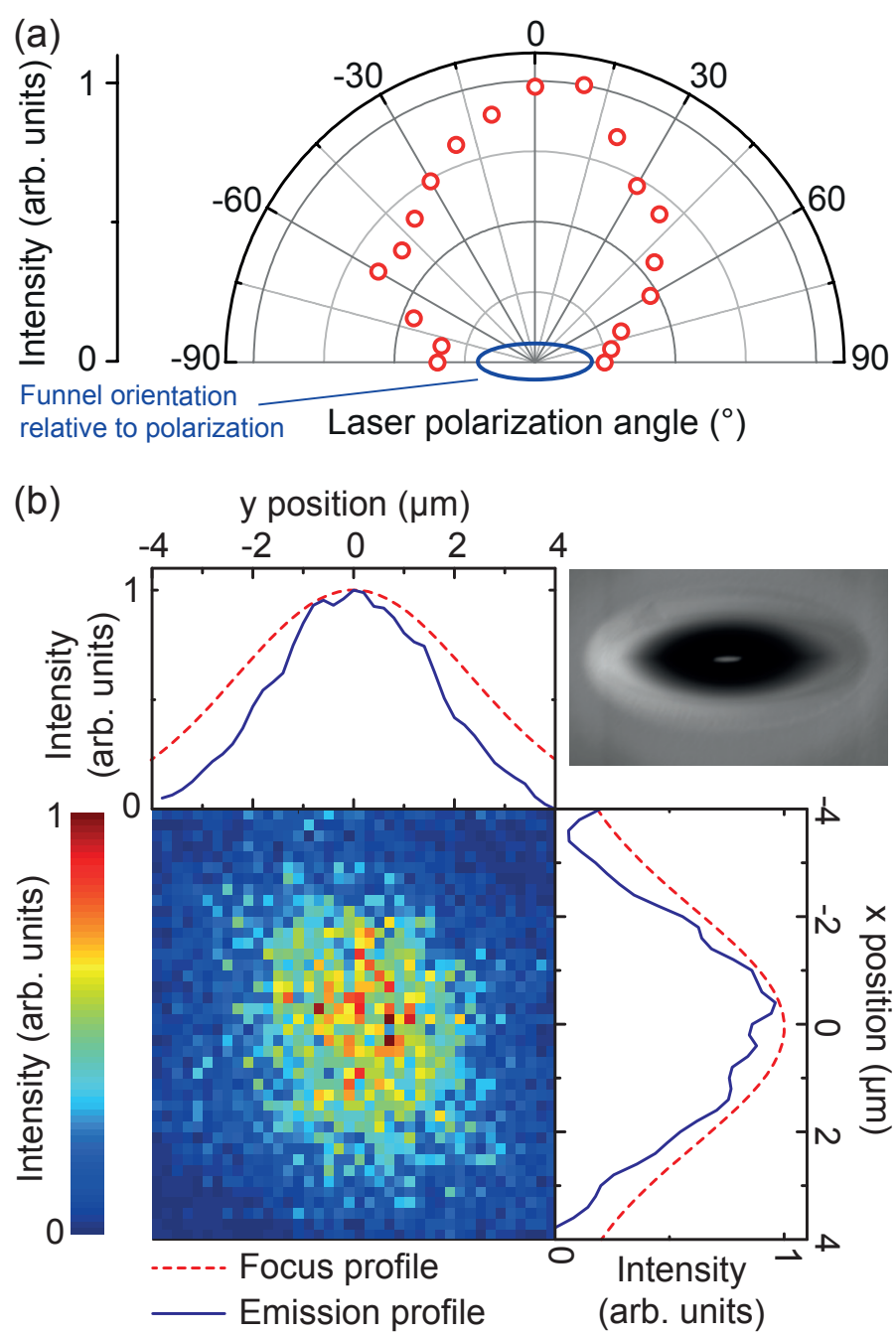

Figure 4.3: (a) Intensity of the 104.8-nm argon line as a function of incident linear laser polarization. Polarization parallel to the minor axis leads to maximum fluorescent emission. (b) Emission map obtained for argon gas. The EUV fluorescence is recorded as the waveguide (cf. inset) is scanned relative to the focal spot. The averaged profiles in the $x$ and $y$ directions (solid blue) both show a significantly narrowed width compared with the focal laser profiles (dashed red), which is caused by spatial mode overlap in conjunction with the emission nonlinearity.

conductivity has advantages over the silver-coated semiconductor taper previously used. Concerning the dimensions of the waveguide exit apertures [here several waveguides with minor (major) axes of typically about $50 \mathrm{~nm}(400 \mathrm{~nm})$ compared with $100 \mathrm{~nm}(200 \mathrm{~nm})$ in Ref. [26]], we believe that the details of the aperture geometry are less critical for the overall volume in which strong-field conditions can be achieved, as long as substantial field compression in the waveguide is present. Instead, the exit aperture is expected to 
strongly influence the overall spectral transmittance and diffraction properties. Importantly, the field-enhanced volume in these structures is assumed to span at least several hundred nanometers before the end of the taper, i.e., multiple EUV wavelengths. Thus, even for incoherent fluorescent emission, wavelength-dependent divergence angles (previously interpreted as evidence for HHG [26]) are expected.

We note that the absence of HHG is not caused by insufficient local intensities, as is evident by the strong contributions from ionic transitions in our measured spectra. Substantial ionization is found even in neon with an ionization energy of $21.5 \mathrm{eV}$, exceeding the energy of 13 laser photons. Furthermore, the observation of a predominance of incoherent emission is consistent with our previous studies on plasmonic bow-tie antennas, in which near-field strengths generally allowing for HHG were verified by utilizing the intensity-dependent atomic line emission spectral fingerprint [81]. By applying this field gauging concept to the waveguide measurements, sufficient local peak intensities above several tens of $\mathrm{TW} / \mathrm{cm}^{2}$ are found. However, considering the field-enhanced volumes in both types of structures, severe limitations on high harmonic signal generation arise from the nanometric confinement of the source and the resulting insufficient coherent radiation buildup. Estimates on the relevant scalings are discussed in Refs. [28, 81, 69]. Specifically, a quadratic scaling of the HHG yield with propagation length poses an important constraint in nanoscale geometries. Reference measurements using amplified laser pulses allow for an estimate of relative HHG to fluorescence signal levels [cf. Eq. (1) in Ref. [81]]. In this comparison, diffraction of potential HHG also has to be considered. The total power of high harmonic radiation generated in the waveguide $\left(L_{\text {nano }} \approx 500 \mathrm{~nm}\right)$ is expected to be 2 orders of magnitude below that of fluorescence emitted into the $\pm 1.2^{\circ}$ detection angle. Diffraction of potential coherent radiation at the exit aperture further reduces the intensity in the forward direction by at least another 2 orders of magnitude. Thus, based on the signals presented in Fig. 4.2(a), we could expect to count less than one HHG photon per second and nanometer, which is below the presently detectable limits, and far below previously reported values. Supporting these considerations, an independent estimate for waveguide structures has projected the high harmonic power to be at least 8 orders of magnitude lower than that achieved in cavity measurements $[69,96]$.

It seems clear that our findings present a serious setback for current approaches to realize compact nanoscale sources of high harmonic radiation for attosecond spectroscopy. Nonetheless, it is apparent that the strong-field regime is reached by means of field enhancement in these nanostructures. The long-term stability and reproducibility of the 
highly nonlinear EUV emission allows for further discovery in detailed investigations of the waveguide excitation and radiation mechanisms under such conditions.

To this end, we have carried out an analysis of the intensity and pressure dependencies of the emission for different gases. First, we find that as function of incident intensityparticularly for higher pressures - the fluorescent intensity exhibits a steep threshold behavior [displayed in Fig. 4.2(b)]. This indicates a density-dependent plasma formation in the waveguide that is most likely triggered by cascaded (for example collisional) excitation and ionization processes involving multiple excited atoms, ions, or electrons. Moreover, at higher pressures, we find a pronounced and reproducible intensity hysteresis, i.e., a situation in which the fluorescent signal at a given incident intensity depends on the excitation history of the system. Figures 4.4(a) and (b) display these intensity hysteresis curves for two different waveguide structures. Specifically, after having applied high intensity, substantial fluorescence can be maintained (upper branch) upon significantly decreasing the intensity to levels, at which hardly any fluorescence is detected when measured in conditions starting from low intensities (lower branch). In atomic gases, bistable behavior is a characteristic feature of complex plasma dynamics, which can manifest itself as hysteresis in the plasma properties upon cycling various physical parameters. The familiar effect of maintaining a gas discharge for reduced voltages after ignition is just one example of such hystereses [97, 98, 99, 100].

The present observations constitute a new kind optical bistability [101] within metal nanostructures [102, 103, 104], which warrants further investigation. As expected for a bistable plasma effect, we find that it strongly depends on the gas species and pressure. In order to quantify the hysteresis, in Fig. 4.4(d), we evaluate the area enclosed by the two branches, normalized to the maximum fluorescent signal [Fig. 4.4(c)]. The prominence of the bistability is found to increase more rapidly with increasing pressures for xenon compared with neon. Moreover, whereas it decreases beyond 150 mbar pressure in xenon, neon displays increasing hysteresis throughout the measured pressure range. The earlier onset of hysteresis for xenon is understood by considering that it has both a lower ionization threshold and a larger atomic cross section for scattering events than neon. This results in reaching a critical excitation density for cascaded plasma formation at lower pressures.

A quantitative modeling of the plasma dynamics within the waveguide is outside of the scope of the present work, so that we give a qualitative description of the types of processes that may contribute to the observed bistability. Generally, the fact that a hysteresis 


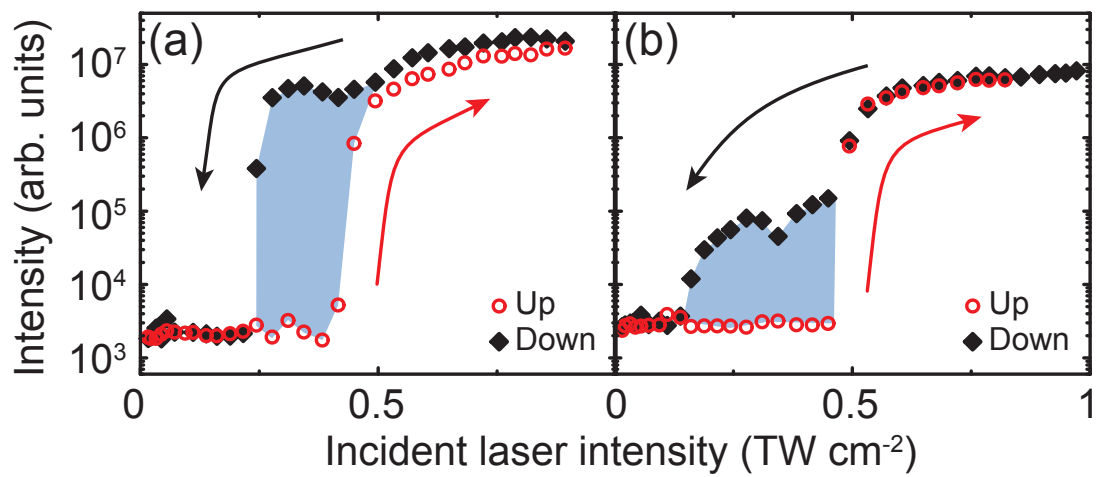

(c)

(d)
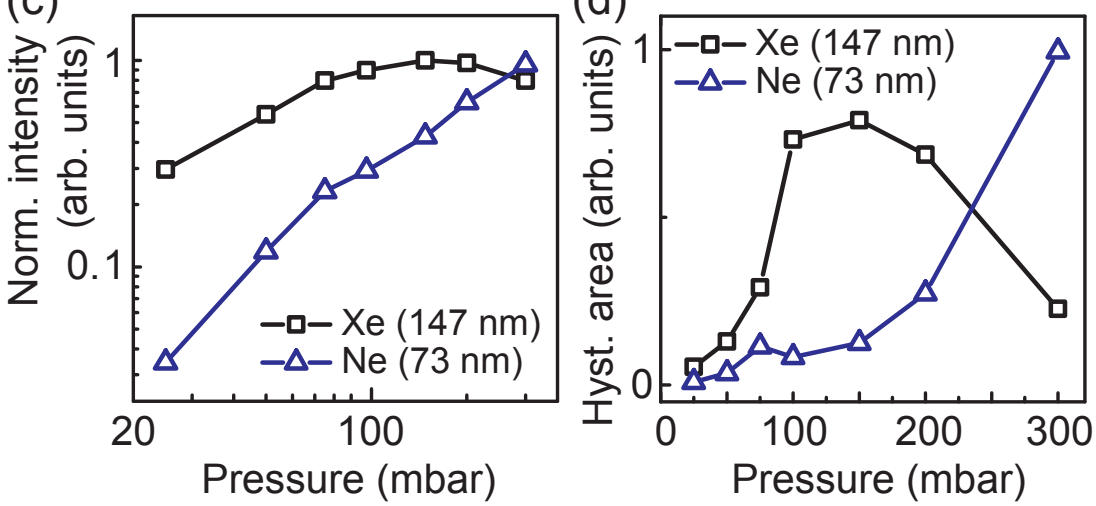

Figure 4.4: (a),(b) Intensity-dependent strength of the 104.8-nm argon line excited in two different waveguides (pressure: 200 mbar). A pronounced intensity hysteresis is observed. (c) Pressure-dependent intensities of two prominent xenon and neon lines. (d) Degree of bistability in xenon and neon as a function of gas pressure, measured as the normalized area enclosed by the hysteresis curves [cf. shaded areas in (a) and (b)].

is observed demonstrates that upon arrival of a laser pulse, there is residual excitation left within the waveguide from the previous excitation. After the laser pulse period of approximately $12 \mathrm{~ns}$, the state of the system will be composed of partially decayed populations of excited states in neutral atoms and ions, free electrons, as well as thermal excitation of the gas and waveguide walls. As thermal transport in the bulk waveguide is rather efficient, the likely most relevant forms of residual excitation are long-lived atomic and ionic states. The reduced ionization energy of such states then causes a higher ionization rate than without prior excitation. An excitation memory alone, however, is not sufficient for the development of a bistability. Instead, the bistable behavior is caused by a collective aspect of the plasma dynamics, which influences the global state of the system and depends in a highly nonlinear way on the density of excited atoms or electrons.

Whereas other potential plasma effects could contribute to the observed bistability, the 
nonlinear pressure dependence of the hysteresis and the fact that accelerated electrons from strong-field photoionization with energies up to several tens of electronvolts are present in significant density suggest that multistep collisional excitations may be the origin of the bistability, as in discharge plasmas [98]. Moreover, multiphoton and strongfield photoemission from the waveguide walls $[13,63,14]$ will provide additional electrons capable of collisional excitation. We have found that upon moderate waveguide modification by using incident powers in excess of $200 \mathrm{~mW}$, the steepness of the threshold slope could be influenced, potentially by introducing roughness and thus altering the contributions from surface-emitted electrons. A more detailed theoretical examination of the plasma bistability based on coupled rate equations $[105,98]$ will be the subject of future work.

In conclusion, we have demonstrated the formation of a submicron noble-gas plasma within tapered waveguides by the observation of extreme-ultraviolet fluorescence. The results illustrate that such tapered plasmonic waveguides are not suitable for efficient nanostructure-enhanced HHG, although sufficient local intensities are achieved. We believe that coherent radiation buildup is limited by the nanoscopic generation length, which is orders of magnitude smaller than in other state-of-the-art HHG concepts [96, 19, 106, 78, 49]. Nonetheless, the observed strong EUV fluorescence and the exhibited optical bistability indicate novel aspects of strong-field physics in conjunction with nonlinear plasmonics. Further studies will elucidate the different roles of surface-mediated, collisional and direct excitation processes within the waveguides. We believe that the possibility to induce highly nonlinear processes in these devices may lead to applications in the areas of EUV near-field imaging, lithography and spectroscopy. The identified bistability of the EUV emission indicates cascaded excitation processes and presents a new link between nanostructure plasmonics and laser-induced surface plasma physics.

We thank M. Duwe, B. Abel, V. Radisch, B. Schröder, and S. Schäfer for helpful discussions. Financial support by the Deutsche Forschungsgemeinschaft (DFG-ZUK 45/1 and SFB 755) is gratefully acknowledged. 


\section{Chapter 5}

\section{General discussion}

In this Section the main results presented in Chapters 2-4 are briefly summarized and discussed. An Outlook sketches perspectives for further investigations of the novel nonlinear effects and illustrates possible applications for the EUV emission obtained from waveguide nanostructures.

\subsection{Summary}

The present thesis critically revisits nanostructure-enhanced EUV light generation processes in atomic gas. Among other things, the possibility of efficient plasmon-enhanced HHG under the previously reported conditions [24, 26, 27] is discussed. Chapter 2 briefly comments on the findings of Kim et al. [24] and presents first experimental evidence for the predominance of incoherent EUV fluorescence over high harmonic generation in bow-tie nanoantennas. A fundamental physical argument concerning the reduced coherent buildup of radiation in the small nanostructure-enhanced generation volume explains the observations and interpretation.

Chapter 3 provides a more detailed examination of EUV light generation enhanced in bow-tie nanoantennas. Additional measurements using different antenna dimensions also led to the exclusive observation of EUV fluorescence without any sign of HHG. The demonstration of fifth-order harmonic generation intrinsic to the nanostructures is a new finding in itself and also indicates that the setup is accurately aligned for the detection of potential coherent HHG contributions in the EUV signal. Sufficient local intensities are verified by applying a novel means to gauge local strong fields in plasmonic nanostructures. Moreover, time-dependent durability measurements show that the structures 
do remain intact for at least several hours of illumination at local peak intensities up to $60 \mathrm{TW} / \mathrm{cm}^{2}$. By evaluating conventional HHG reference measurements, an estimate for potentially detectable HHG contributions relative to the observed fluorescence signal is given. This estimate reveals that the high harmonic signal level must be several orders of magnitude below that of the observed EUV fluorescence. Whereas such signal levels are potentially detectable under special conditions, it is obvious that the incoherent fluorescent photons will dominate the emitted radiation for the given geometries.

Chapter 4 deals with strong-field EUV light generation processes within tapered hollow waveguide nanostructures and discusses the feasibility of HHG in such an approach. Again, the exclusive observation of fluorescent radiation (even from neon gas) indicates an apparent conflict with the previous demonstrations of nanostructure-enhanced HHG [26, 27]. The signal levels of the observed EUV fluorescence for argon and xenon gas are about one order of magnitude larger than those recorded in the bow-tie measurements. This increase is caused by the roughly ten times larger generation volume in the waveguides compared to that in the bow-tie experiments. Together with the observation of an intensity-dependent bistability of the EUV signal — due to the formation of a nanoplasma in the waveguides - the results give entirely new insights into strong-field gas excitation in plasmonic nanostructures.

\subsection{Plasmonic enhancement of HHG revisited}

The following discussion concludes the findings regarding nanostructure-enhanced HHG in bow-tie antennas and tapered hollow waveguides. Since the sole experimental realizations of such approaches are reported in just three publications [24, 26, 27], which all arise from the same group of scientists, we aim to consider the plausibility of these reports based on the results obtained in the present work. Thus, it is instructive to first recap the main findings regarding the feasibility of efficient HHG in nanostructure-based implementations.

First of all, the results of this thesis clearly illustrate that extreme-ultraviolet light generation in plasmonic nanostructures is generally feasible. However, it is also revealed that the underlying generation process is not high-order harmonic up-conversion of laser radiation, as previously claimed $[24,26]$. Each of the measured spectra in the present experiments clearly shows that the observed EUV emission stems exclusively from atomic and ionic fluorescence, which is caused by multiphoton excitation and strong-field ion- 
ization of the noble gas atoms. Furthermore, no signature of coherent high harmonic radiation was found, although sufficient intensities for HHG were reached in the nanostructures, and the experiments were repeated numerous times with different bow-ties and waveguides for various laser intensities, gas species, and pressures. Very recently, an independent study involving a similar implementation with bow-tie nanoantennas has resulted in the clear observation of fluorescent emission, with no conclusive evidence of high harmonic radiation [107].

As already discussed in Chapters 2-4, the reason for this observation is the fact that efficient plasmon-enhanced HHG is not feasible under the examined conditions. The physical argument concerns the coherent nature of the HHG process. In short, the nanoscopic interaction distance between the atoms and the enhanced near-fields leads to an insufficient coherent radiation buildup given its quadratic length-dependent scaling (see section 1.1); therefore, high harmonic yields are negligible. On the other hand, incoherent fluorescence scales only linearly with generation length and is enhanced enough to be readily observed, as shown in this work.

In agreement with this physical consideration, the estimates carried out in Chapters 3 and 4 illustrate that the potential harmonic signal levels in such nanostructure-enhanced implementations are expected to be orders of magnitude below those of the observed fluorescent emission. In other words, only a few high harmonic photons per second are expected to be countable in these measurements. Taking into account the detection efficiency of the experimental setup and an emission solid angle of $\pm 1.2^{\circ}$, this yield corresponds to the generation of at most $10^{2}$ and $10^{3}$ coherent photons per second at each harmonic order in forward direction for the bow-tie and waveguide experiments, respectively. These findings are in contradiction to the previous reports, which described count rates in excess of $10^{8}$ and $10^{9}$ harmonic photons per second at each harmonic order in their bow-tie and waveguides measurements, respectively [24, 26]. It was recently acknowledged by the authors that the photon numbers they reported do not represent count rates, but in fact projected generation rates by considering a detection efficiency of $10^{-5}$ for their setup [27] (see also Reply by Kim et al. in the Appendix A); however, the contradiction is still present, as illustrated in table 5.1. Here, the high harmonic generation rates reported by Kim and Park et al. [24, 26] are compared to the values estimated in Chapters 3 and 4. Additionally, the table contains expected generation rates, which were estimated independently in a recent comment on nanostructure-enhanced HHG [69]. These theoretically extrapolated values are in good agreement with those estimated experimen- 


\begin{tabular}{lcc}
\hline \hline & \multicolumn{2}{c}{ HHG photon generation rate $\left(\mathrm{s}^{-1}\right)$} \\
& Bow-ties & Waveguide \\
\hline Kim/Park et al. $[24,26]$ & $10^{8}$ & $10^{9}$ \\
Estimation [this work] & $\sim 10^{2}$ & $\sim 10^{3}$ \\
Extrapolation [69] & $10^{2}-10^{3}$ & $10^{3}-10^{4}$ \\
\hline \hline
\end{tabular}

Table 5.1: Reported generation rates per harmonic order for plasmon-enhanced HHG in bow-tie and waveguide nanostructures (by Kim and Park et al. [24, 26]) in comparison to expected HHG rates estimated in this work and values taken from Ref. [69].

tally in the present work. For both types of nanostructures, the expected high harmonic yields are up to six orders of magnitude lower than those reported in the two previous studies $[24,26]$.

Taking into account these estimations together with our experimental results, it is evident that the previous reports about plasmon-enhanced HHG should be re-evaluated. Shortly after our reports, also the authors of Refs. [24] and [26] are reconsidering their initial interpretations. In a follow-up article, Kim et al. state [27]: "It is also important to note that there may be a contribution from ALE as claimed in Ref. [13] [here Ref. [28]]. However, it is practically not possible to isolate the contribution of ALE directly in our EUV spectrum. The reason is that there are too many ALE lines possibly emitted from the ionized Ar atoms in the range of 40-80 nm, with some ALE lines even being in too close proximity to H11-H17 harmonics [...]." Referencing to the graph shown in Fig. 5.1, the Supplement of this article further notes [27]: "The calibrated spectrum clearly shows the harmonic peaks in both the cases, but it is found that the observed peaks are not precisely located at the expected positions of harmonic wavelengths. This may be simply due to the calibration imperfection of our home-built spectrometer. Nonetheless, the discrepancy could be attributable to other reasons such as atomic line emission as claimed in Ref. [13] [here Ref. [28]] [...]."

Whereas a final clarification of this issue might still be expected, the present work already has unequivocally demonstrated that high harmonic generation is exceedingly inefficient and essentially negligible under field-enhanced conditions involving localized surface plasmons in both bow-tie and waveguide nanostructures. A realistic increase in the local atom density in the waveguides by a factor of 10 (a pressure increase of about 5 bar) would probably enable the observation of high harmonic photons; however, even under such high pressure conditions, the high harmonic yield should be only on the 


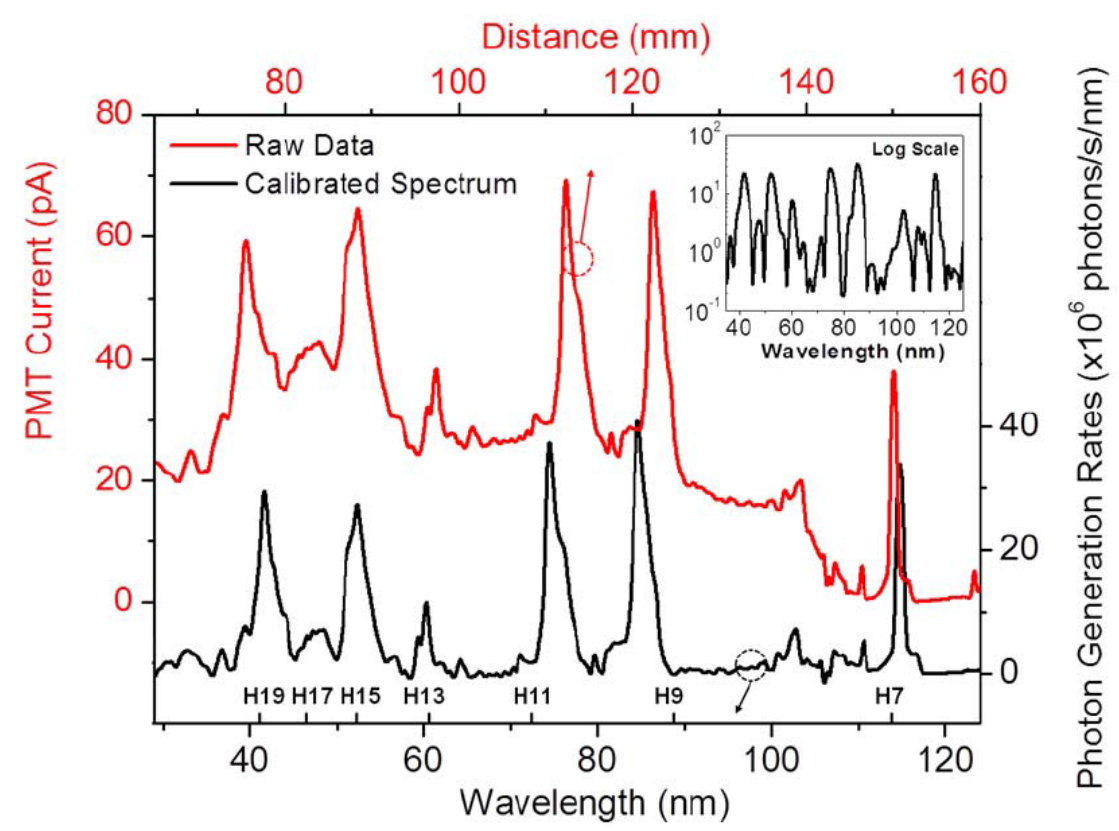

Figure 5.1: Nanostructure-enhanced EUV spectrum taken from the supplement of Ref. [27]. This spectrum is a reproduction of the measurement shown in Ref. [24]. The expected harmonic orders are indicated in the lower axis (H7-H19). (C)2013 Wiley.

order of several hundred photons per second and will still be surpassed by the fluorescent emission.

In consequence, entirely different approaches would be needed to raise the coherent EUV contributions significantly above the levels of the incoherent fluorescence. A possible alternative concept for efficient nanostructure-assisted HHG utilizing propagating surface-plasmon-polariton fields instead of LSPs is provided in the next section. 


\subsection{Concept for efficient plasmon-enhanced HHG}

As described above, HHG is not efficiently produced in implementations that utilize LSPs for field enhancement. To overcome the underlying volume limitations, we envision a different plasmon-enhanced approach, which combines the tapered waveguide geometry with a longer channel-waveguide. Figure 5.2(a) schematically depicts this idea. In this scenario, an incident low-energy laser pulse excites SPP waves at the inner walls of a tapered waveguide similar to those used in Chapter 4. These propagating SPP waves become concentrated at the tapered end of the funnel and are guided into a channel of several micrometers in length. Due to the long propagation distance of the SPP waves at the inner wall of the channel-waveguide, the total field-enhanced volume is drastically increased compared to implementations using LSPs, so that sufficient coherent radiation buildup for HHG may be realized. Together with a high pressure gas supply, this approach could lead to a situation where the high harmonic yield clearly dominates the fluorescent contribution in the EUV emission.

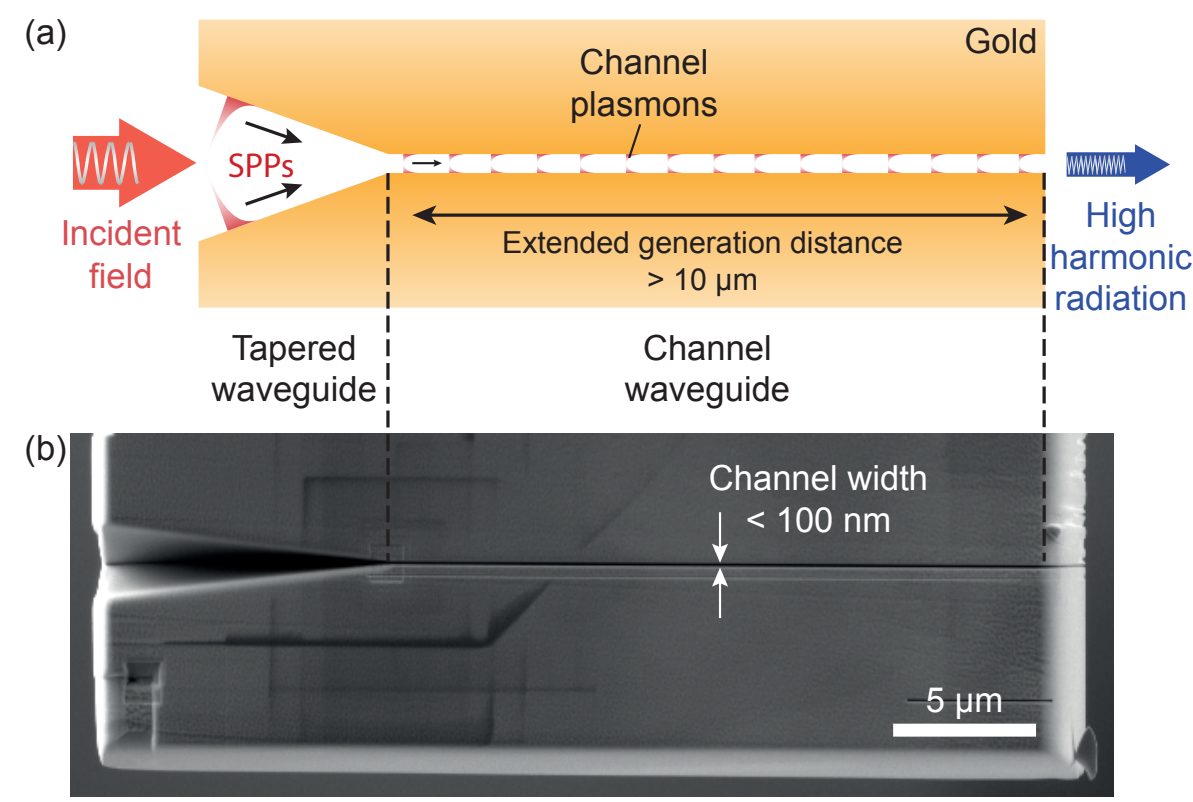

Figure 5.2: Concept for efficient plasmon-enhanced HHG by using SPP waves in a channel waveguide. (a) Schematic illustration of channel-plasmon-enhanced HHG. (b) Scanning electron microscope image of a prototype sample. The gold platform contains a combination of a tapered waveguide and a $30 \mu \mathrm{m}$ long hollow channel, which is $100 \mathrm{~nm}$ wide and few micrometer deep. 
The efficient coupling of channel SPPs - channel-plasmon-polaritons (CPP) - into Vshaped grooves has already been demonstrated and characterized by near-field scanning optical microscopy (NSOM), as shown in Fig. 5.3 [95, 108]. A smooth gold film is deposited on a fused silica substrate and contains V-shaped channels, which have a tailored geometry in terms of opening $\theta$ angle and depth $d$ (see SEM image in Fig. 5.3(a)) ${ }^{1}$. In Fig. 5.3(b), the NSOM image illustrates the near-field distribution and homogeneous propagation of the CPPs within the channel for an excitation wavelength of $1.6 \mu \mathrm{m}$. Here, an efficient coupling of the CPPs depends critically on the geometry of the grooves. Calculations for rectangular gold channels indicate that propagation distances on the order of $200 \mu \mathrm{m}$ are expected for certain CPP modes at $1.55 \mu \mathrm{m}$ wavelength [95], allowing for a sufficient generation length in a HHG scheme with channel plasmons.

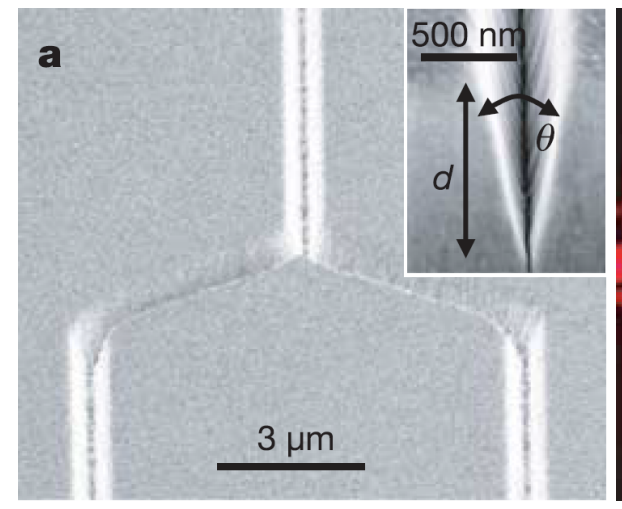

(a) SEM image of a channel waveguide

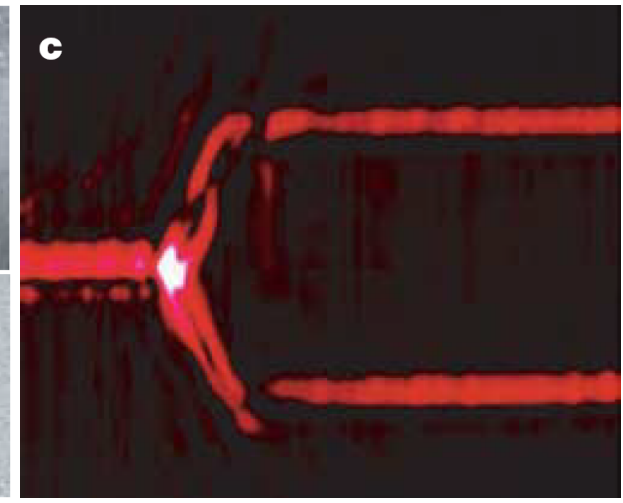

(b) NSOM image

Figure 5.3: Channel-plasmon-polaritons. Results from Ref. [108]. (a) Scanning electron microscope image of the gold film containing V-shaped grooves forming a plasmonic Y-splitter. (b) Near-field scanning optical microscope (NSOM) image displaying the field distribution of the $1.6 \mu \mathrm{m}$ light in the channel waveguide. Images taken from Ref. [108]. (C)2006 npg.

Currently, attempts to realize channel-plasmon-enhanced HHG are in progress and are expected to enable a clear observation of high harmonic signals from nanostructures for the first time. Figure 5.2(b) depicts a prototype sample for such an experiment. A combination of a tapered waveguide with a micrometer-long channel is fabricated via focused ion milling onto a smooth gold platform. Pressure-dependent measurements of the EUV light generation in such samples with differing channel lengths may lead to the observation of the transition from fluorescence to HHG dominated EUV spectra.

\footnotetext{
${ }^{1}$ The Y-splitter configuration of the channels has no further relevance in this context.
} 


\subsection{Outlook}

This section provides a road map for further investigations of the novel nanostructurebased EUV effects found in this study. Specifically, gauging the near-field, surfaceenhanced low-order harmonic generation, and the formation of the bistable waveguide nanoplasma are discussed. A first attempt to explain the bistability in the waveguide plasma in terms of coupled rate equations is also included. Finally, some potential applications for the waveguide-enhanced EUV emission are suggested.

\subsubsection{Gauging of plasmonic near-fields}

One of the unsolved challenges in nano-optics is the determination of absolute field strengths in optical nanostructures by means of a direct gauge. The main problem in this respect is caused by the lack of suitable probes for sensing the nanoscopically confined near-fields. Whereas other properties, for example, the plasmonic resonances of nanoantennas are relatively easy to access via spectroscopy $[52,51,16]$ (see also the dark-field scattering spectra in Fig. 3.2e), the absolute intensity values of local fields are still difficult to extract. Approaches relying on the evaluation of absolute signals, e.g, from twophoton photo-luminescence excited at the metal surface of the nanostructures [60], were suggested in the past. However, such concepts may face diverse problems in the form of uncertainties about nanostructure-specific changes of the signal emission properties due to quenching, diffraction, or absorption causing inaccuracies in the obtained intensity values. Other methods utilizing relative signal levels are generally more reliable in this context and were already demonstrated for nanostructures which exhibit moderate field enhancements [76]. In this latter report, relative luminescent contributions from near-field excited colloid quantum dots were harnessed to find an absolute intensity scale. However, due to the dimensions of quantum dots (about $10 \mathrm{~nm}$ in diameter), this approach is restricted to nanostructures with relatively large dimensions and low field enhancements.

In contrast, the gauging method presented in Chapter 3 can be applied to arbitrarily small nanostructures (in terms of field-enhanced volumes) and allows for the sensing of very high local intensities even reaching the destruction threshold of the nanostructures. In analogy to the approach in Ref. [76], the intensity-dependent relative line strengths of the fluorescent atomic emission provide an absolute measure of the near-field enhancement. In the same manner, it is also anticipated that this method can be used to gauge the 
absolute local gas densities, since the relative line strengths of the fluorescence show also a clear pressure dependence (cf. Fig. 3.4c).

In order to make this near-field gauging more accessible and versatile, an important next task is the implementation of this method under ambient conditions. Due to the strong absorption of EUV photons at atmospheric pressure, this goal requires intensitydependent fluorescence lines at longer (e.g. visible) wavelengths. This approach will be the subject of further studies. Many nano-optical applications rely on accurate knowledge of absolute near-field intensities and could, therefore, benefit from this new gauging method.

It should also be noted that the formation of the bistable plasma in the waveguide experiments could have a significant influence on the near-field gauge due to the alternation in intensity-dependence of the fluorescent emission. Such alternations are clearly indicated by the threshold behavior and the strong hysteresis observed in our experiments. However, these plasma effects are only present at high pressures, as evident from Fig. 4.4d. The main reason behind this property is simply the much stronger collisional contribution from free electrons to the atomic excitation process due to a increased gas density; therefore, this gauging method is not applicable at high-pressure conditions. On the other hand, plasma formation in the bow-tie experiments was not observed, most likely because of the much smaller excitation volumes in this scenario. The formation of laser induced-plasma requires an excitation memory together with multi-collisional electron excitation of the gas atoms. Whereas the second requirement is most probably satisfied in the bow-ties, the fast escape of pre-excited atoms and ions from the field-enhanced region (due to simple gas kinetics) prevents plasma formation. In other words, the gas volume is renewed upon arrival of a subsequent exciting laser pulse (see also explanation in Chapter 4).

Beyond the applicability of intensity-dependent spectral characteristics for gauging purposes, the study of nonlinear fluorescent gas excitation within strong fields is very interesting in itself. As an example, Fig. 3.4(d) shows a transition from the multiphoton to a different excitation regime characterized by a linear intensity dependence of the fluorescence line strengths. This and other aspects of the plasmonic EUV light generation will be the subject of further studies. 


\subsubsection{Surface-enhanced low-order harmonic generation}

The nonlinear optical response from metallic nanostructures, including antennas, sharp tips, particles, and rough surfaces, is well investigated and has enabled the observation of light conversion effects like second [70, 3, 4] and third [5, 6] harmonic generation as well as two-photon photo-luminescence $[59,60]$ at the metal surfaces. These observations offer the great potential to study the nonlinear optical properties of the respective bulk metals and surfaces. Exact values for the nonlinear refractive indices or the nonlinear susceptibilities of gold and other materials are already obtainable experimentally. The investigation methods usually rely on $z$-scan absorption measurements or the evaluation of low-order harmonic light generation $[109,110,111,112,113]$. The utilization of the fifth-order harmonic generation in nanostructures, as it is demonstrated here, will offer a new means of investigating nonlinear optical properties of metals and other materials in terms of higher nonlinear orders and shorter wavelengths in the ultraviolet spectral range.

\subsubsection{Optical bistability of the waveguide nanoplasma}

Plasma formation is necessarily linked to a high degree of ionization in an atomic medium and the density of the charged particles_electrons and ions-serves as the key marker for the underlying plasma dynamics which are governed by collisional processes between these particles [114]. Due to the large scattering cross section of fast electrons, electron collisions usually dominate the plasma process. The formation of a gas plasma can be easily induced by electron discharges, which rely on direct or inductive ionization of a gas volume [57, 55, 115, 97, 98]. But intense laser radiation can also be utilized to obtain a plasma through multiphoton or strong-field ionization [30, 116, 117, 118].

In this respect, the waveguide-enhanced EUV fluorescence reported in Chapter 4 indicates a new way to study laser-induced plasma processes at high repetition rates. Specifically, the observed intensity hysteresis in the EUV signal warrants further investigation, and is intended to provide a first approach for a more detailed explanation of optical bistabilities in waveguide nanoplasmas. In order to derive a model based on coupled rate equations, it is instructive to look at similar effects in other types of plasma experiments.

Specifically, for inductively-coupled (IC) discharge plasmas, bistabilities have been studied for a long time, resulting in a large number of reports [97, 98, 99, 100, 120, 121, 119, 122, 123]. For example, Fig. 5.4(a) reprints a plasma bistability measurement taken from Ref. [97]. Here, the emission yield from an argon plasma shows a strong 


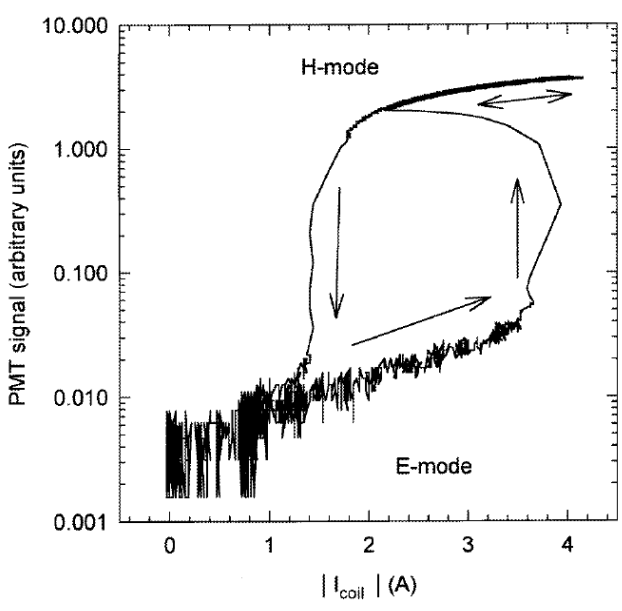

(a) Plasma emission hysteresis

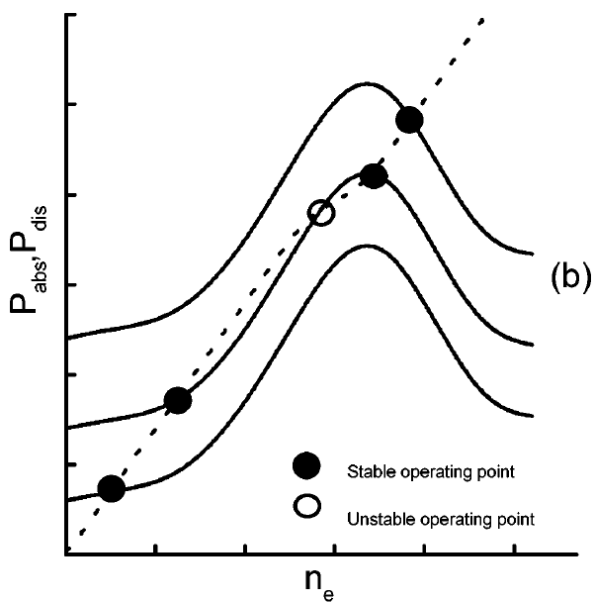

(b) Power balance diagram

Figure 5.4: Bistability in a inductively-coupled gas discharge plasma. (a) Emission intensity of the argon plasma lines at $419.8 \mathrm{~nm}$ and $420.0 \mathrm{~nm}$ wavelength as a function of the coil current. The arrows denote the time evolution of the trace. (b) Power balance diagram for an inductive discharge. Shown are the dissipated (dotted line) and absorbed (solid lines) power as a function of free electron density for three different coil currents. Stable and unstable operation points are indicated with filled and open circles, respectively. Graphs are taken from refs. [97] and [119]. (C) 1996/1999 IOP Publishing Ltd.

hysteresis as a function of the coil current. Such bistabilities are commonly explained by considering power balance diagrams, which compare the dissipated with the absorbed power in the IC discharge plasma [119]. In Fig. 5.4(b), a typical power balance diagram shows the dissipated power $P_{d i s}\left(n_{e}\right)$ (dotted) and the absorbed power $P_{a b s}\left(n_{e}\right)$ (solid) as functions of the free electron density $n_{e}$. Equilibrium in plasma generation is reached, if $P_{d i s}\left(n_{e}\right)=P_{a b s}\left(n_{e}\right)$, and the free electron density becomes constant. The corresponding intersections (filled circles) indicate the stable operation points of the plasma. Due to the nonlinear behavior of the absorbed power $P_{a b s}\left(n_{e}\right)$, which is plotted here for three different coil currents, monostable operation conditions are present for small or large currents, whereas bistable conditions only occur at intermediate currents. The hysteresis shown in Fig. 5.4(a) becomes clear under consideration of such a power balance diagram. Therefore, starting from small coil currents, the system remains in the operation point at lower electron densities (low hysteresis branch) until the monostable condition at large coil currents results in a plasma at higher electron densities. This change in turn causes the fluorescence strength to increase by orders of magnitude. After this ignition process, the high electron density conditions can be maintained (upper hysteresis branch) until 
monostable conditions at sufficiently small coil currents force the system back into the operation point at low electron densities.

Following this above explanation, it is clear that the free-electron density is the key aspect of the plasma generation process. Specifically, stable operation conditions are characterized by a constant electron density, which implies a zero rate change $d n_{e} / d t=0$. Taking this into account, the bistability of the waveguide plasma can be discussed by evaluating the rate of change of the electron density $d n_{e} / d t$ as a function of electron density $n_{e}$ itself. The rate of change is affected by several excitation and relaxation channels, which include multiphoton and strong-field ionization, together with direct or stepwise electron-collisional excitations, as well as collisional de-excitation and fluorescence. Fig. 5.5 depicts several of these processes, together with

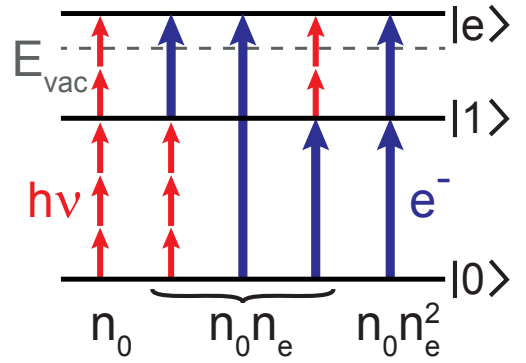

Figure 5.5: Ionization paths. the dependence of the electron production rate on the atom density in the ground state $\left(n_{0}\right)$ and the free electron density $\left(n_{e}\right)$. Both direct and stepwise ionization via the numerous accessible atomic states (labeled by $|1\rangle$ ) contribute. Based on this consideration the following two rate equations determine the change of the electron density.

$$
\begin{gathered}
\frac{d n_{e}}{d t}=\Gamma_{0 e} n_{0}+\Gamma_{1 e} n_{1}+S_{1 e} n_{1} n_{e}+S_{0 e} n_{0} n_{e}-\gamma_{e} n_{e} \\
\frac{d n_{1}}{d t}=\Gamma_{01} n_{0}+S_{01} n_{0} n_{e}-\gamma_{1} n_{1} .
\end{gathered}
$$

Here, the coefficients $\Gamma_{x y}$ and $S_{x y}$ represent the transition probabilities from state $x$ to $y$ for photon-driven and collisionally-exited electrons, respectively. The coefficients for the de-excitation processes and the fluorescence are summarized in $\gamma_{e}$ and $\gamma_{1}$.

The coupled rate equations 5.1 and 5.2 lead to a density dependence (up to third-order) of the rate change $d n_{e} / d t$, considering the mass density conservation $\rho=n_{0}+n_{1}+n_{e}$. Thus, purely light-induced excitations and those involving up to one electron scattering event scale linearly and quadratically, respectively, in the electron densities, whereas stepwise ionization mechanisms involving two collisions provide the $n_{e}^{3}$ scaling, which is necessary for the bistability, as described below. 


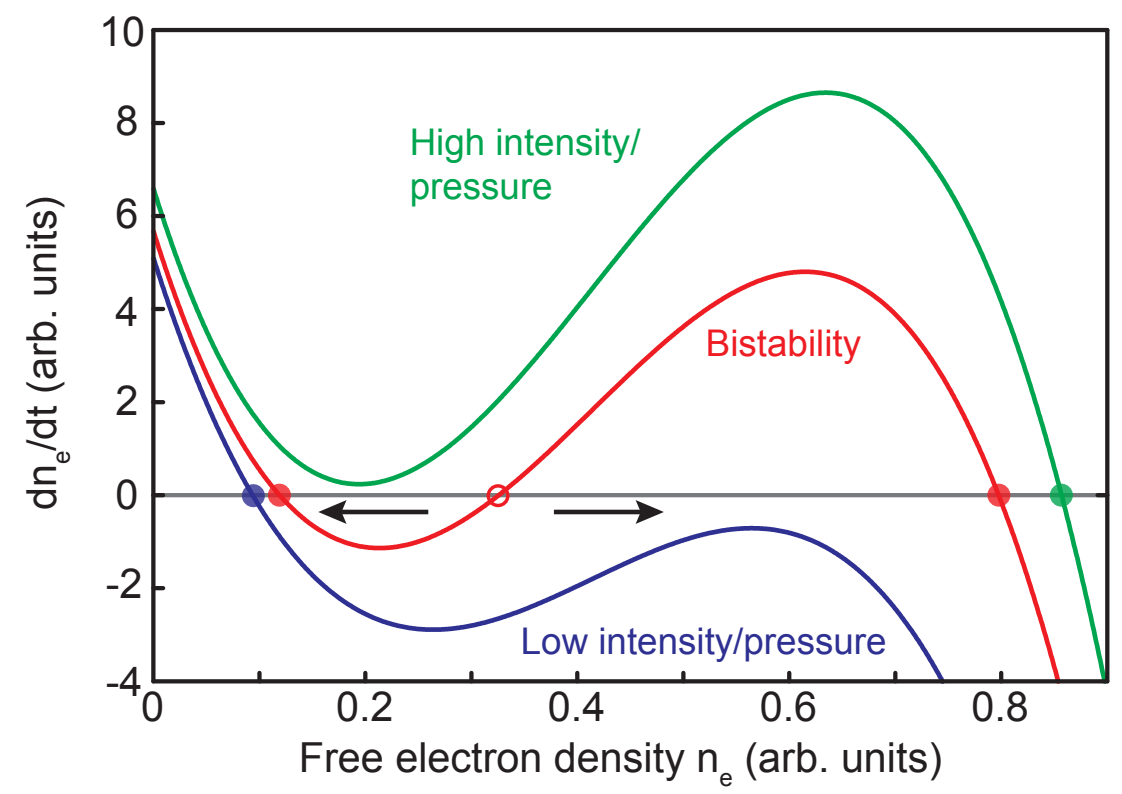

Figure 5.6: Illustration of the appearance of bistability based on stepwise excitation processes. Ionization rate in the plasma as a function of free electron density for the laser intensities and/or gas pressures below (blue), within (red) and above (green) the bistable regime. Filled and open circles indicate stable and unstable zero-rate change conditions, respectively.

A qualitative evaluation of $d n_{e} / d t$ for different laser intensities leads to the bistability diagram shown in Fig. 5.6. Here, monostable conditions (green/blue for low/high intensities), are characterized by a positive rate at zero density and negative rate at high density, with a single intersection at the stability point $\left(d n_{e} / d t=0\right)$. For the bistable intensity regime (red) two stable operation points are present, and the plasma exhibits either low or high electron densities, depending on the excitation history of the system. This characteristic results in the observation of the pronounced intensity hysteresis shown in Fig. 5.7. Similar considerations should also hold for differing gas densities, and the experimental verification of a pressure hysteresis is in progress. Furthermore, by choosing realistic values for the coefficients in equations 5.1 and 5.2 it should be possible to obtain quantitative information about the optical bistability in the waveguide nanoplasma. Such an analysis will be the subject of future studies, resulting in a deeper understanding of the observed plasma dynamics.

However, additional electron contributions due to multiphoton and strong-field photoemission from the waveguide walls $[13,63,14]$ are expected to influence the exhibited plasma dynamics and, thus, will have to be included in the above model. For example 
this affects the mass density conservation mentioned above. Figure 5.7 shows the effect of these additional electron contributions to the shape of the intensity hysteresis in the waveguide EUV fluorescence. In contrast to the very step hysteresis in Fig. 5.7(a), which was measured with a fresh waveguide having a smooth surface, the same waveguide with increased surface roughness (due to laser damage) exhibits a completely different hysteresis (cf. Fig. 5.7(b)). In particular, the earlier onset of the EUV emission at lower incident intensities indicates strong contributions of photoelectrons from the metal surface. This reduction of the plasma excitation threshold can be understood considering the metal's work function (about $5 \mathrm{eV}$ for gold), which is much smaller that the ionization energy of the gas atoms. Further investigation of these surface mediated contributions are in progress.

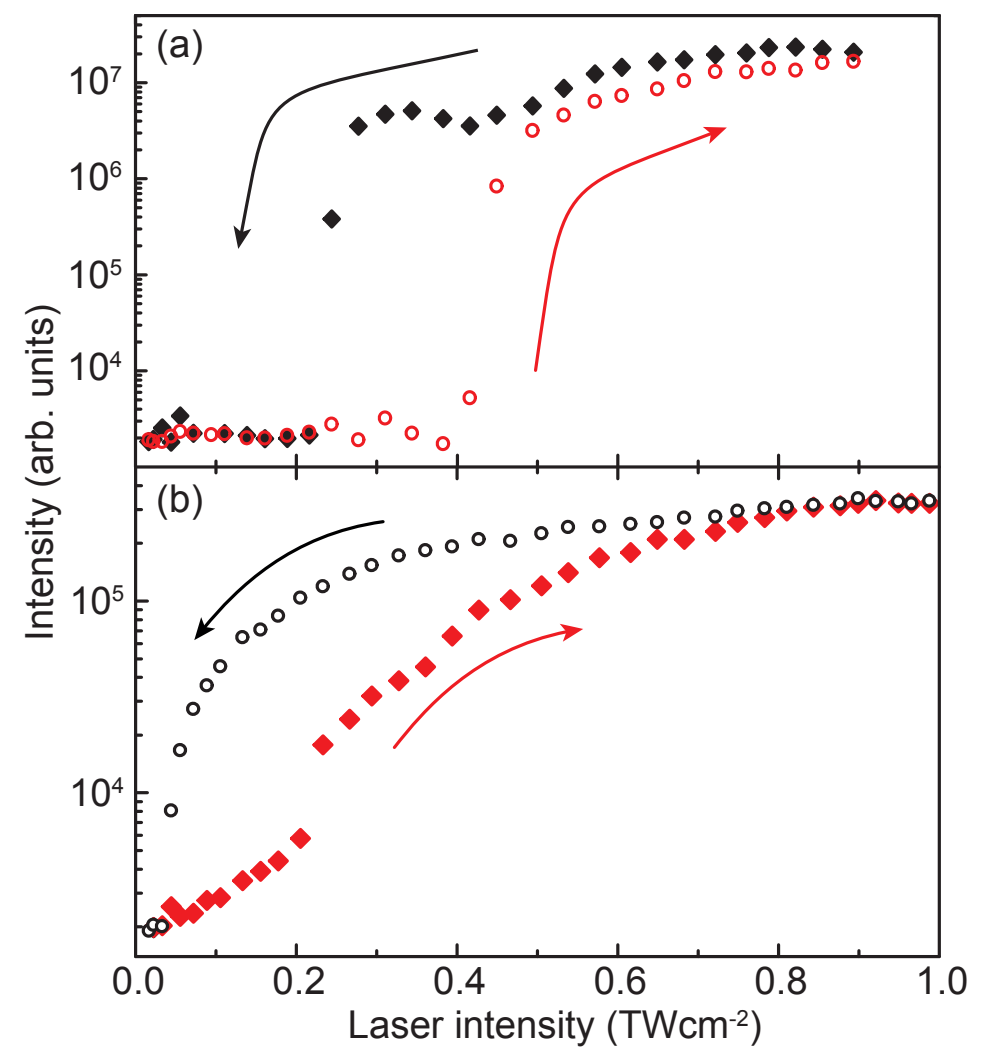

Figure 5.7: Intensity hysteresis from a fresh and moderately modified waveguide. a) Hysteresis measurement using a fresh waveguide with smooth inner surface right after the fabrication process. Same data as shown in Fig. 4.4(a). (b) Hysteresis measurement for the same waveguide as in (a), but with moderately modified surface roughness caused by illumination with laser intensities in the excess of $1 \mathrm{TW} / \mathrm{cm}^{2}$. 


\subsubsection{Future applications for EUV fluorescence}

Besides the further study of strong-field gas excitation in plasmonic nanostructures, it is also intended to utilize the very stable fluorescence yield-particularly from the tapered hollow waveguides - in experiments that do not necessarily require temporally coherent light. Taking into account the detection efficiency of the setup, a total generation rate of up to $10^{10}$ fluorescence photons per second is expected at the exit of an optimal waveguide structure. These photon fluxes are large enough to be considered in experimental implementations based on scanning near-field techniques or nanoscale coherent diffractive imaging methods. The schemes in Figs. 5.8(a) and (b) illustrate possible concepts for scanning near-field optical microscopy/lithography and lensless imaging, respectively. A striking advantage of the waveguide-based light source is its small size and the great flexibility to fabricate the structures on any kind of metal tip or cantilever. This enables a very compact design of the respective experiments and allows us to bring the EUV source as close as desired to the sample (see Fig. 5.8).
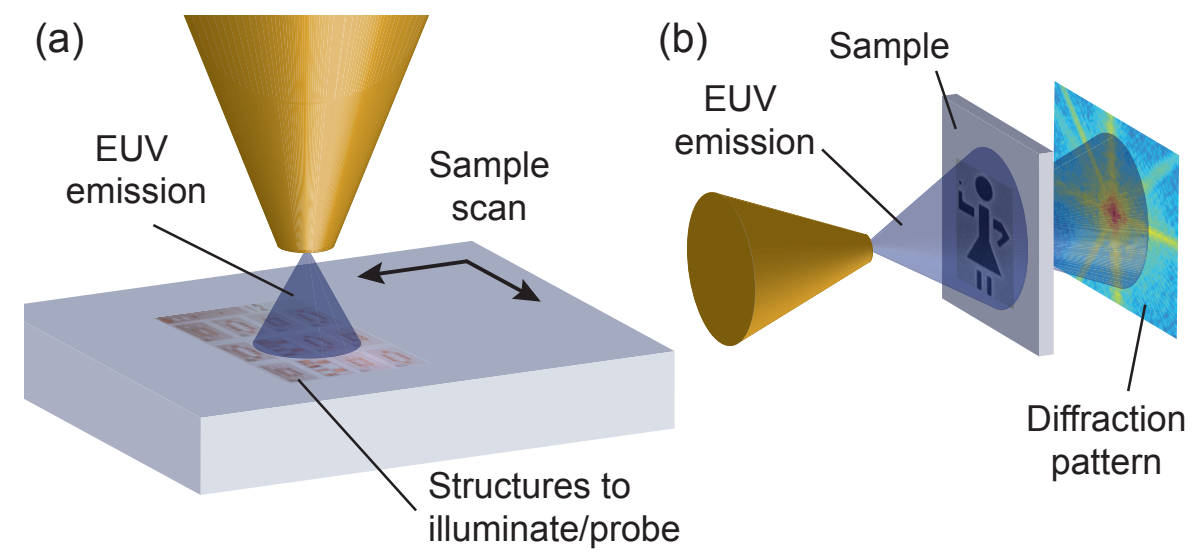

Figure 5.8: Possible applications for the waveguide-enhanced EUV emission. a) Scheme for EUV scanning near-field optical microscopy or lithography. The exit of the waveguide source is placed in close proximity to a sample, which can be scanned in lateral directions. b) Scheme for lensless imaging. The EUV radiation emitted from the waveguide source passes the sample containing a phase or intensity object, and a diffraction pattern is recorded with a camera behind the sample. The pictures of the sample and the diffraction pattern are both adapted from Ref. [21]. 


\section{Appendix A}

\section{Kim et al. reply}

S. Kim, J. Jin, Y.-J. Kim, I.-Y. Park, Y. Kim, and S.-W. Kim

Nature 485, E2-E3 (2012)

DOI: $10.1038 /$ nature 10978

Kim et al. wrote in their reply to the comment in Chapter 2:

"Sivis et al. showed [28] spectral data of extreme ultraviolet (EUV) emission from gasexposed bow-ties, claiming high predominance of atomic line emission (ALE) of neutral and ionized gas atoms in contradiction to our data [24, 58] of high harmonic generation (HHG). This is not the first time the signature of ALE has been identified in conventional HHG spectral data [78]. The two distinct phenomena, ALE and HHG, are not mutually exclusive but coexistent when gaseous atoms are illuminated by strong-field laser pulses.

The feasibility of nanostructure-enhanced HHG has been proved already by several theoretical studies conducted independently [46, 65, 66, 67]. However, no experimental demonstration has yet been reported except for our HHG data [24, 58] from bow-ties. The main reason may be inferred from the durability problem we encountered with bow-ties patterned with Au on a sapphire substrate. The thin-film bow-ties began to degrade, not abruptly, but starting gradually then continuing at a fast rate, soon after being exposed to

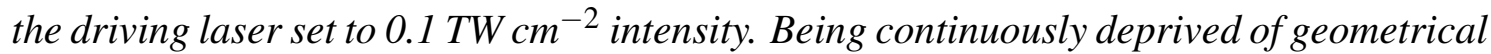
accuracy by thermal damage coupled with optical breakdown, even new bow-ties gave out detectable HHG signals during a short lifetime, which was often missed.

We do not agree with Sivis et al. [28] that $N_{\text {nano }} / N_{\text {conv }} \approx 10^{-8}$. Our calculation reveals that the ratio reaches $10^{-6}$; we calculate the number of gas atoms to be $\sim 8 \times 10^{4}$ with a total interaction volume of $60 \mathrm{~nm} \times 350 \mathrm{~nm} \times 350 \mathrm{~nm} \times 150$ bow-ties at 115 torr pressure. 
This brings the conversion efficiency of nano-HHG for the seventh harmonic (H7) to be $\sim 10^{-4}$ times that of conventional HHG. Given that the conversion efficiency of $H 7$ is $\sim 10^{-5}$ in conventional HHG [124], the efficiency value of $10^{-9}$ for the harmonic shown in our data is realistic. No consideration was given to the possible enhancement of harmonic yield attributable to inhomogeneous distribution of plasmonic field intensity [67].

The photon-count of our data [24, 58] was estimated by measuring the output current of the photomultiplier tube (PMT) used to scan the raw spectral data, and at the same time taking into account all the individual functional efficiencies of the hardware components involved in our experiment. This projected estimation of photon-count led to a noise floor of $\sim 10^{6}$ photons $s^{-1}$, which appeared rather high due to electrical noise in the PMT current measurement.

The linewidth became narrowed during post-processing, both for deconvolution of the slit size placed before the PMT and also for data averaging through repetitive measurements to reduce electric noise. Long plasmonic field decay might have caused the linewidth narrowing [66], which is however not proved yet. No attention was paid to the second or higher-order diffraction signals because they were buried below the noise floor in our measurement; the grating efficiency for the second order diffraction was one order of magnitude less than that for the first order. Besides, the peak amplitudes of higher harmonics were also about one order of magnitude less than that of H7.

Further work continued after our Letter [24] brought us the conclusion that bow-ties are not an ideal tool for experimental demonstration of nano-HHG. Instead, we found that three-dimensional waveguides [26] hollowed out in an ellipsoidal funnel shape on a bulk metal substrate are a good alternative. The funnel waveguide permits stable, consistent generation of higher harmonics up to the 43rd order using xenon gas with improved immunity to optical and thermal damage. Investigation is underway to verify the coherence of the EUV radiation from the funnel waveguide." 


\section{Bibliography}

[1] S. Nie and S. R. Emory. Probing Single Molecules and Single Nanoparticles by Surface-Enhanced Raman Scattering. Science, 275(5303):1102-1106, February 1997. Cited on p. 1, 29

[2] S. Kühn, U. Håkanson, L. Rogobete, and V. Sandoghdar. Enhancement of SingleMolecule Fluorescence Using a Gold Nanoparticle as an Optical Nanoantenna. Physical Review Letters, 97(1):017402, July 2006. Cited on p. 1, 29

[3] A. Bouhelier, M. Beversluis, A. Hartschuh, and L. Novotny. Near-Field SecondHarmonic Generation Induced by Local Field Enhancement. Physical Review Letters, 90(1):013903, January 2003. Cited on p. 1, 29, 60

[4] C. Neacsu, G. Reider, and M. Raschke. Second-harmonic generation from nanoscopic metal tips: Symmetry selection rules for single asymmetric nanostructures. Physical Review B, 71(20):201402, May 2005. Cited on p. 1, 29, 60

[5] M. Lippitz, M. A. van Dijk, and M. Orrit. Third-harmonic generation from single gold nanoparticles. Nano letters, 5(4):799-802, April 2005. Cited on p. 1, 30, 44, 60

[6] T. Hanke, G. Krauss, D. Träutlein, B. Wild, R. Bratschitsch, and A. Leitenstorfer. Efficient Nonlinear Light Emission of Single Gold Optical Antennas Driven by Few-Cycle Near-Infrared Pulses. Physical Review Letters, 103(25):257404, December 2009. Cited on p. 1, 25, 28, 30, 44, 60

[7] C. Ropers, D. R. Solli, C. P. Schulz, C. Lienau, and T. Elsaesser. Localized Multiphoton Emission of Femtosecond Electron Pulses from Metal Nanotips. Physical Review Letters, 98(4):043907, January 2007. Cited on p. 1, 30 
[8] A. Kubo, K. Onda, H. Petek, Z. Sun, Y. S. Jung, and H. K. Kim. Femtosecond imaging of surface plasmon dynamics in a nanostructured silver film. Nano letters, 5(6):1123-7, June 2005. Cited on p. 1, 30

[9] J. A. Schuller, E. S. Barnard, W. Cai, Y. C. Jun, J. S. White, and M. L. Brongersma. Plasmonics for extreme light concentration and manipulation. Nature materials, 9(3):193-204, March 2010. Cited on p. 1, 15

[10] D. K. Gramotnev and S. I. Bozhevolnyi. Plasmonics beyond the diffraction limit. Nat Photon, 4(2):83-91, February 2010. Cited on p. 1, 15

[11] M. Kauranen and A. V. Zayats. Nonlinear plasmonics. Nature Photonics, 6(11):737-748, November 2012. Cited on p. 1, 15

[12] R. Bormann, M. Gulde, A. Weismann, S. V. Yalunin, and C. Ropers. Tip-Enhanced Strong-Field Photoemission. Physical Review Letters, 105(14):147601, September 2010. Cited on p. 1, 30, 41

[13] M. Krüger, M. Schenk, and P. Hommelhoff. Attosecond control of electrons emitted from a nanoscale metal tip. Nature, 475(7354):78-81, July 2011. Cited on p. 1, $30,41,50,63$

[14] G. Herink, D. R. Solli, M. Gulde, and C. Ropers. Field-driven photoemission from nanostructures quenches the quiver motion. Nature, 483(7388):190-193, March 2012. Cited on p. 1, 30, 41, 50, 63

[15] D. J. Park, B. Piglosiewicz, S. Schmidt, H. Kollmann, M. Mascheck, and C. Lienau. Strong Field Acceleration and Steering of Ultrafast Electron Pulses from a Sharp Metallic Nanotip. Physical Review Letters, 109(24):244803, December 2012. Cited on p. 1, 30, 41, 42, 44

[16] P. Dombi, A. Hörl, P. Rácz, I. Márton, A. Trügler, J. R. Krenn, and U. Hohenester. Ultrafast strong-field photoemission from plasmonic nanoparticles. Nano letters, 13(2):674-8, February 2013. Cited on p. 1, 41, 58

[17] S. Zherebtsov, T. Fennel, J. Plenge, E. Antonsson, I. Znakovskaya, A. Wirth, O. Herrwerth, F. Süßmann, C. Peltz, I. Ahmad, S. A. Trushin, V. Pervak, S. Karsch, M. J. J. Vrakking, B. Langer, C. Graf, M. I. Stockman, F. Krausz, E. Rühl, and 
M. F. Kling. Controlled near-field enhanced electron acceleration from dielectric nanospheres with intense few-cycle laser fields. Nature Physics, 7(8):656-662, April 2011. Cited on p. 1, 30, 41

[18] A. McPherson, G. Gibson, H. Jara, U. Johann, T. S. Luk, I. A. McIntyre, K. Boyer, and C. K. Rhodes. Studies of multiphoton production of vacuum-ultraviolet radiation in the rare gases. Journal of the Optical Society of America B, 4(4):595, April 1987. Cited on p. 2

[19] X. Li, A. L'Huillier, M. Ferray, L. Lompré, and G. Mainfray. Multiple-harmonic generation in rare gases at high laser intensity. Physical Review A, 39(11):57515761, June 1989. Cited on p. 2, 11, 12, 13, 20, 27, 50

[20] P. Corkum. Plasma perspective on strong field multiphoton ionization. Physical Review Letters, 71(13):1994-1997, September 1993. Cited on p. 2, 10, 11, 30, 36

[21] R. L. Sandberg, C. Song, P. W. Wachulak, D. A. Raymondson, A. Paul, B. Amirbekian, E. Lee, A. E. Sakdinawat, C. La-O-Vorakiat, M. C. Marconi, C. S. Menoni, M. M. Murnane, J. J. Rocca, H. C. Kapteyn, and J. Miao. High numerical aperture tabletop soft $\mathrm{x}$-ray diffraction microscopy with 70 -nm resolution. Proceedings of the National Academy of Sciences of the United States of America, 105(1):24-7, January 2008. Cited on p. 2, 9, 65

[22] P. B. Corkum and F. Krausz. Attosecond science. Nature Physics, 3(6):381-387, June 2007. Cited on p. 2, 9, 41

[23] P. H. Bucksbaum. The future of attosecond spectroscopy. Science (New York, N.Y.), 317(5839):766-9, August 2007. Cited on p. 2, 9

[24] S. Kim, J. Jin, Y.-J. Kim, I.-Y. Park, Y. Kim, and S.-W. Kim. High-harmonic generation by resonant plasmon field enhancement. Nature, 453(7196):757-60, June 2008. Cited on p. 2, 3, 4, 5, 17, 25, 26, 27, 28, 30, 41, 42, 51, 52, 53, 54, 55, 67,68

[25] M. I. Stockman. Attosecond physics: an easier route to high harmony. Nature, 453(7196):731-3, June 2008. Cited on p. 3 
[26] I.-Y. Park, S. Kim, J. Choi, D.-H. Lee, Y.-J. Kim, M. F. Kling, M. I. Stockman, and S.-W. Kim. Plasmonic generation of ultrashort extreme-ultraviolet light pulses. Nature Photonics, 5(11):677-681, October 2011. Cited on p. 4, 17, 18, 19, 27, 30, $41,42,43,44,46,47,51,52,53,54,68$

[27] I.-Y. Park, J. Choi, D.-H. Lee, S. Han, S. Kim, and S.-W. Kim. Generation of EUV radiation by plasmonic field enhancement using nano-structured bowties and funnel-waveguides. Annalen der Physik, 525(1-2):87-96, February 2013. Cited on p. $4,51,52,53,54,55$

[28] M. Sivis, M. Duwe, B. Abel, and C. Ropers. Nanostructure-enhanced atomic line emission. Nature, 485(7397):E1-E2, May 2012. Cited on p. 5, 30, 32, 36, 41, 42, $47,54,67$

[29] S. Kim, J. Jin, Y.-J. Kim, I.-Y. Park, Y. Kim, and S.-W. Kim. Reply. Nature, 485:E2-E3, 2012. Cited on p. 5, 30

[30] P. Laporte, N. Damany, and H. Damany. Pulsed-laser-generated rare-gas plasma as a light source in the vacuum ultraviolet. Optics letters, 12(12):987-9, December 1987. Cited on p. 8,60

[31] J. K. Crane, M. D. Perry, D. Strickland, S. Herman, and R. W. Falcone. Coherent and incoherent XUV emission in helium and neon, laser-driven plasmas. IEEE Transactions on Plasma Science, 21(1):82-89, 1993. Cited on p. 8

[32] R. Coffee and G. Gibson. vuv fluorescence from selective high-order multiphoton excitation of $\mathrm{N}_{2}$. Physical Review A, 69(5):053407, May 2004. Cited on p. 8, 9

[33] T. Brabec and F. Krausz. Intense few-cycle laser fields: Frontiers of nonlinear optics. Reviews of Modern Physics, 72(2):545-591, April 2000. Cited on p. 9, 10, $12,30,36,39$

[34] P. M. Paul, E. S. Toma, P. Breger, G. Mullot, F. Auge, P. Balcou, H. G. Muller, and P. Agostini. Observation of a train of attosecond pulses from high harmonic generation. Science (New York, N.Y.), 292(5522):1689-92, June 2001. Cited on p. 9 
[35] M. Hentschel, R. Kienberger, C. Spielmann, G. A. Reider, N. Milosevic, T. Brabec, P. Corkum, U. Heinzmann, M. Drescher, and F. Krausz. Attosecond metrology. Nature, 414(6863):509-13, November 2001. Cited on p. 9

[36] A. L'Huillier, P. Balcou, and L. Lompré. Coherence and resonance effects in highorder harmonic generation. Physical Review Letters, 68(2):166-169, January 1992. Cited on p. 10

[37] E. S. Toma, P. Antoine, A. de Bohan, and H. G. Muller. Resonance-enhanced high-harmonic generation. Journal of Physics B: Atomic, Molecular and Optical Physics, 32(24):5843-5852, December 1999. Cited on p. 10

[38] J. Krause, K. Schafer, and K. Kulander. High-order harmonic generation from atoms and ions in the high intensity regime. Physical Review Letters, 68(24):35353538, June 1992. Cited on p. 11

[39] S. A. Maier. Plasmonics: Fundamentals and Applications. Springer Science, New York, 2007. Cited on p. 14, 15, 18, 44

[40] G. Mie. Beiträge zur Optik trüber Medien, speziell kolloidaler Metallösungen. Annalen der Physik, 330(3):377-445, 1908. Cited on p. 14

[41] M. I. Stockman. Nanoplasmonics: past, present, and glimpse into future. Nano letters, 19(22):22029-22106, October 2011. Cited on p. 15

[42] P. Biagioni, J.-S. Huang, and B. Hecht. Nanoantennas for visible and infrared radiation. Reports on progress in physics. Physical Society (Great Britain), 75(2):024402, February 2012. Cited on p. 15, 16

[43] Heinz Raether. Surface plasmons on smooth surfaces. Springer, 1988. Cited on p. 15

[44] P G Etchegoin, E C Le Ru, and M Meyer. An analytic model for the optical properties of gold. The Journal of chemical physics, 125(16):164705, October 2006. Cited on p. 16

[45] H. Fischer and O. J. F. Martin. Engineering the optical response of plasmonic nanoantennas. Optics express, 16(12):9144-54, June 2008. Cited on p. 16, 25, 28, 29 
[46] A. Husakou, S.-J. Im, and J. Herrmann. Theory of plasmon-enhanced high-order harmonic generation in the vicinity of metal nanostructures in noble gases. Physical Review A, 83(4):043839, April 2011. Cited on p. 17, 30, 36, 42, 67

[47] M. I. Stockman. Nanofocusing of Optical Energy in Tapered Plasmonic Waveguides. Physical Review Letters, 93(13):137404, September 2004. Cited on p. 18, 19,44

[48] C. Ropers, C. C. Neacsu, T. Elsaesser, M. Albrecht, M. B. Raschke, and C. Lienau. Grating-coupling of surface plasmons onto metallic tips: a nanoconfined light source. Nano letters, 7(9):2784-8, September 2007. Cited on p. 18, 19

[49] T. Popmintchev, M.-C. Chen, P. Arpin, M. M. Murnane, and H. C. Kapteyn. The attosecond nonlinear optics of bright coherent X-ray generation. Nature Photonics, 4(12):822-832, December 2010. Cited on p. 20, 50

[50] D. A. Genov, A. K. Sarychev, V. M. Shalaev, and A. Wei. Resonant Field Enhancements from Metal Nanoparticle Arrays. Nano Letters, 4(1):153-158, January 2004. Cited on p. 25, 29

[51] J. Merlein, M. Kahl, A. Zuschlag, A. Sell, A. Halm, J. Boneberg, P. Leiderer, A. Leitenstorfer, and R. Bratschitsch. Nanomechanical control of an optical antenna. Nature Photonics, 2(4):230-233, March 2008. Cited on p. 25, 28, 29, 58

[52] D. P. Fromm, A. Sundaramurthy, P. J. Schuck, G. Kino, and W. E. Moerner. GapDependent Optical Coupling of Single Bowtie Nanoantennas Resonant in the Visible. Nano Letters, 4(5):957-961, May 2004. Cited on p. 25, 29, 58

[53] K. Li, M. I. Stockman, and D. J. Bergman. Self-Similar Chain of Metal Nanospheres as an Efficient Nanolens. Physical Review Letters, 91(22):227402, November 2003. Cited on p. 25, 29

[54] L. Minnhagen. Accurately Measured and Calculated Ground-Term Combinations of Ar II. Journal of the Optical Society of America, 61(9):1257, September 1971. Cited on p. 25, 26

[55] L. Minnhagen. Spectrum and the energy levels of neutral argon, Ar I. Journal of the Optical Society of America, 63(10):1185, October 1973. Cited on p. 25, 26, 60 
[56] J. E. Sansonetti and W. C. Martin. Handbook of Basic Atomic Spectroscopic Data. Journal of Physical and Chemical Reference Data, 34(4):1559, 2005. Cited on p. $25,26,31,32,44,45$

[57] J. C. Boyce. The Spectra of Xenon in the Extreme Ultraviolet. Physical Review, 49(10):730-732, May 1936. Cited on p. 26, 60

[58] S. Kim, I.-Y. Park, J. Choi, and S.-W. Kim. Progress in Ultrafast Intense Laser Science, volume 6, pages 129-144. Springer, 2010. Cited on p. 28, 67, 68

[59] M. Beversluis, A. Bouhelier, and L. Novotny. Continuum generation from single gold nanostructures through near-field mediated intraband transitions. Physical Review B, 68(11):115433, September 2003. Cited on p. 30, 60

[60] P. J. Schuck, D. P. Fromm, A. Sundaramurthy, G. S. Kino, and W. E. Moerner. Improving the Mismatch between Light and Nanoscale Objects with Gold Bowtie Nanoantennas. Physical Review Letters, 94(1):017402, January 2005. Cited on p. $30,58,60$

[61] P. Mühlschlegel, H.-J. Eisler, O. J. F. Martin, B. Hecht, and D. W. Pohl. Resonant optical antennas. Science (New York, N.Y.), 308(5728):1607-9, June 2005. Cited on p. 30

[62] M. Schenk, M. Krüger, and P. Hommelhoff. Strong-Field Above-Threshold Photoemission from Sharp Metal Tips. Physical Review Letters, 105(25):257601, December 2010. Cited on p. 30, 41

[63] P. Rácz and P. Dombi. Nonponderomotive electron acceleration in ultrashort surface-plasmon fields. Physical Review A, 84(6):063844, December 2011. Cited on p. $30,41,50,63$

[64] S. V. Yalunin, G. Herink, D. R. Solli, M. Krüger, P. Hommelhoff, M. Diehn, A. Munk, and C. Ropers. Field localization and rescattering in tip-enhanced photoemission. Annalen der Physik, 525(1-2):L12-L18, February 2013. Cited on p. 30,41

[65] A. Husakou, F. Kelkensberg, J. Herrmann, and M. J. J. Vrakking. Polarization gating and circularly-polarized high harmonic generation using plasmonic enhance- 
ment in metal nanostructures. Optics Express, 19(25):25346, November 2011. Cited on p. 30, 42, 67

[66] S. L. Stebbings, F. Süßmann, Y.-Y. Yang, A. Scrinzi, M. Durach, A. Rusina, M. I. Stockman, and M. F. Kling. Generation of isolated attosecond extreme ultraviolet pulses employing nanoplasmonic field enhancement: optimization of coupled ellipsoids. New Journal of Physics, 13(7):073010, July 2011. Cited on p. 30, 42, 67, 68

[67] I. Yavuz, E. A. Bleda, Z. Altun, and T. Topcu. Generation of a broadband xuv continuum in high-order-harmonic generation by spatially inhomogeneous fields. Physical Review A, 85(1):013416, January 2012. Cited on p. 30, 42, 67, 68

[68] C. Kern, M. Zürch, J. Petschulat, T. Pertsch, B. Kley, T. Käsebier, U. Hübner, and C. Spielmann. Comparison of femtosecond laser-induced damage on unstructured vs. nano-structured Au-targets. Applied Physics A, 104(1):15-21, May 2011. Cited on p. 30,33

[69] M. B. Raschke. High-harmonic generation with plasmonics: feasible or unphysical? Annalen der Physik, 525(3):A40-A42, March 2013. Cited on p. 30, 47, 53, 54

[70] Y. R. Shen. Surface properties probed by second-harmonic and sum-frequency generation. Nature, 337(6207):519-525, February 1989. Cited on p. 31, 60

[71] T. Tsang. Third- and fifth-harmonic generation at the interfaces of glass and liquids. Physical review. A, 54(6):5454-5457, December 1996. Cited on p. 31, 44

[72] S. Augst, D. Strickland, D. D. Meyerhofer, S. L. Chin, and J. Eberly. Tunneling ionization of noble gases in a high-intensity laser field. Physical Review Letters, 63(20):2212-2215, November 1989. Cited on p. 33

[73] W. Bryan, S. Stebbings, E. English, T. Goodworth, W. Newell, J. McKenna, M. Suresh, B. Srigengan, I. Williams, I. Turcu, J. Smith, E. Divall, C. Hooker, and A. Langley. Geometry- and diffraction-independent ionization probabilities in intense laser fields: Probing atomic ionization mechanisms with effective intensity matching. Physical Review A, 73(1):013407, January 2006. Cited on p. 33 
[74] W. A. Bryan, S. L. Stebbings, J. McKenna, E. M. L. English, M. Suresh, J. Wood, B. Srigengan, I. C. E. Turcu, J. M. Smith, E. J. Divall, C. J. Hooker, a. J. Langley, J. L. Collier, I. D. Williams, and W. R. Newell. Atomic excitation during recollision-free ultrafast multi-electron tunnel ionization. Nature Physics, 2(6):379-383, May 2006. Cited on p. 33

[75] G. Lawrence. Radiance Lifetimes in the Resonance Series of Ar I. Physical Review, 175(1):40-44, November 1968. Cited on p. 34

[76] H. Aouani, S. Itzhakov, D. Gachet, E. Devaux, T. W. Ebbesen, H. Rigneault, D. Oron, and J. Wenger. Colloidal quantum dots as probes of excitation field enhancement in photonic antennas. ACS nano, 4(8):4571-8, August 2010. Cited on p. 35,58

[77] R. Jones, K. Moll, M. Thorpe, and J. Ye. Phase-Coherent Frequency Combs in the Vacuum Ultraviolet via High-Harmonic Generation inside a Femtosecond Enhancement Cavity. Physical Review Letters, 94(19):193201, May 2005. Cited on p. 38

[78] C. Gohle, T. Udem, M. Herrmann, J. Rauschenberger, R. Holzwarth, H. A. Schuessler, F. Krausz, and T. W. Hänsch. A frequency comb in the extreme ultraviolet. Nature, 436(7048):234-7, July 2005. Cited on p. 38, 50, 67

[79] C.-T. Chiang, A. Blättermann, M. Huth, J. Kirschner, and W. Widdra. High-order harmonic generation at $4 \mathrm{MHz}$ as a light source for time-of-flight photoemission spectroscopy. Applied Physics Letters, 101(7):071116, 2012. Cited on p. 38

[80] N. Milosevic, A. Scrinzi, and T. Brabec. Numerical Characterization of High Harmonic Attosecond Pulses. Physical Review Letters, 88(9):093905, February 2002. Cited on p. 39

[81] M. Sivis, M. Duwe, B. Abel, and C. Ropers. Extreme-ultraviolet light generation in plasmonic nanostructures. Nature Physics, 9(5):304-309, March 2013. Cited on p. $41,42,44,47$

[82] S. V. Yalunin, M. Gulde, and C. Ropers. Strong-field photoemission from surfaces: Theoretical approaches. Physical Review B, 84(19):195426, November 2011. Cited on p. 41 
[83] M. F. Ciappina, J. A. Pérez-Hernández, T. Shaaran, J. Biegert, R. Quidant, and M. Lewenstein. Above-threshold ionization by few-cycle spatially inhomogeneous fields. Physical Review A, 86(2):023413, August 2012. Cited on p. 41

[84] T. Shaaran, M. F. Ciappina, and M. Lewenstein. Quantum-orbit analysis of highorder-harmonic generation by resonant plasmon field enhancement. Physical Review A, 86(2):023408, August 2012. Cited on p. 42

[85] M. F. Ciappina, S. S. Aćimović, T. Shaaran, J. Biegert, R. Quidant, and M. Lewenstein. Enhancement of high harmonic generation by confining electron motion in plasmonic nanostructures. Optics Express, 20(24):26261, November 2012. Cited on p. 42

[86] T. Shaaran, M. F. Ciappina, and M. Lewenstein. Estimating the plasmonic field enhancement using high-order harmonic generation: the role of the field inhomogeneity. Journal of Modern Optics, 59(19):1634-1639, November 2012. Cited on p. 42

[87] M. F. Ciappina, T. Shaaran, and M. Lewenstein. High order harmonic generation in noble gases using plasmonic field enhancement. Annalen der Physik, 525(1-2):97106, February 2013. Cited on p. 42

[88] B. Fetić, K. Kalajdžić, and D. B. Milošević. High-order harmonic generation by a spatially inhomogeneous field. Annalen der Physik, 525(1-2):107-117, February 2013. Cited on p. 42

[89] Y.-Y. Yang, A. Scrinzi, A. Husakou, Q.-G. Li, S. L. Stebbings, F. Süßmann, H.-J. Yu, S. Kim, E. Rühl, J. Herrmann, X.-C. Lin, and M. F. Kling. High-harmonic and single attosecond pulse generation using plasmonic field enhancement in ordered arrays of gold nanoparticles with chirped laser pulses. Optics Express, 21(2):2195, January 2013. Cited on p. 42

[90] I. Yavuz. Gas population effects in harmonic emission by plasmonic fields. Physical Review A, 87(5):053815, May 2013. Cited on p. 42

[91] M. Labardi, M. Zavelani-Rossi, D. Polli, G. Cerullo, M. Allegrini, S. De Silvestri, and O. Svelto. Characterization of femtosecond light pulses coupled to hollow- 
pyramid near-field probes: Localization in space and time. Applied Physics Letters, 86(3):031105, 2005. Cited on p. 44

[92] C. Mihalcea, W. Scholz, S. Werner, S. Münster, E. Oesterschulze, and R. Kassing. Multipurpose sensor tips for scanning near-field microscopy. Applied Physics Letters, 68(25):3531, 1996. Cited on p. 44

[93] M. Hentschel, T. Utikal, H. Giessen, and M. Lippitz. Quantitative modeling of the third harmonic emission spectrum of plasmonic nanoantennas. Nano letters, 12(7):3778-82, July 2012. Cited on p. 44

[94] W. L. Barnes, A. Dereux, and T. W. Ebbesen. Surface plasmon subwavelength optics. Nature, 424(6950):824-30, August 2003. Cited on p. 44

[95] S. I. Bozhevolnyi, V. S. Volkov, E. Devaux, and T. W. Ebbesen. Channel PlasmonPolariton Guiding by Subwavelength Metal Grooves. Physical Review Letters, 95(4):046802, July 2005. Cited on p. 44, 57

[96] A. Cingöz, D. C. Yost, T. K. Allison, A. Ruehl, M. E. Fermann, I. Hartl, and J. Ye. Direct frequency comb spectroscopy in the extreme ultraviolet. Nature, 482(7383):68-71, February 2012. Cited on p. 47, 50

[97] U. Kortshagen, N. D. Gibson, and J. E. Lawler. On the E - H mode transition in RF inductive discharges. Journal of Physics D: Applied Physics, 29(5):1224-1236, May 1996. Cited on p. 48, 60, 61

[98] M. M. Turner and M. A. Lieberman. Hysteresis and the E-to-H transition in radiofrequency inductive discharges. Plasma Sources Science and Technology, 8(2):313-324, May 1999. Cited on p. 48, 50, 60

[99] K. N. Ostrikov, S. Xu, and S. Lee. Bistability and Hysteresis in Inductively Coupled Plasmas. Physica Scripta, 62(2-3):189-193, August 2000. Cited on p. 48, 60

[100] Y. Chen, Z.-G. Guo, X.-M. Zhu, Z.-G. Mao, and Y.-K. Pu. Spatially resolved optical emission spectroscopy investigation of $\mathrm{E}$ and $\mathrm{H}$ modes in cylindrical inductively coupled plasmas. Journal of Physics D: Applied Physics, 40(17):5112-5116, September 2007. Cited on p. 48, 60 
[101] H. M. Gibbs. Optical Bistability: Controlling Light with Light. Academic, New York, 1985. Cited on p. 48

[102] G. Wurtz, R. Pollard, and A. Zayats. Optical Bistability in Nonlinear SurfacePlasmon Polaritonic Crystals. Physical Review Letters, 97(5):057402, August 2006. Cited on p. 48

[103] Y. Shen and G. P. Wang. Optical bistability in metal gap waveguide nanocavities. Optics express, 16(12):8421-6, June 2008. Cited on p. 48

[104] G. Wang, H. Lu, X. Liu, Y. Gong, and L. Wang. Optical bistability in metalinsulator-metal plasmonic waveguide with nanodisk resonator containing Kerr nonlinear medium. Applied optics, 50(27):5287-90, September 2011. Cited on p. 48

[105] V. S. Vorob'ev and S. V. Maksimenko. Kinetics of the formation of a surface laser plasma in the absence of surface damage. Soviet Journal of Quantum Electronics, 17(5):664-669, May 1987. Cited on p. 50

[106] E. A. Gibson, A. Paul, N. Wagner, R. Tobey, D. Gaudiosi, S. Backus, I. P. Christov, A. Aquila, E. M. Gullikson, D. T. Attwood, M. M. Murnane, and H. C. Kapteyn. Coherent soft $\mathrm{x}$-ray generation in the water window with quasi-phase matching. Science (New York, N.Y.), 302(5642):95-8, October 2003. Cited on p. 50

[107] N. Pfullmann, C. Waltermann, M. Noack, S. Rausch, T. Nagy, C. Reinhardt, M. Kovačev, V. Knittel, R. Bratschitsch, D. Akemeier, A. Hütten, A. Leitenstorfer, and U. Morgner. Bow-tie nano-antenna assisted generation of extreme ultraviolet radiation. New Journal of Physics, 15(9):093027, September 2013. Cited on p. 53

[108] S. I. Bozhevolnyi, V. S. Volkov, E. Devaux, J.-Y. Laluet, and T. W. Ebbesen. Channel plasmon subwavelength waveguide components including interferometers and ring resonators. Nature, 440(7083):508-11, March 2006. Cited on p. 57

[109] D. D. Smith, Y. Yoon, R. W. Boyd, J. K. Campbell, L. A. Baker, R. M. Crooks, and M. George. Z-Scan Measurement of the Nonlinear Absorption of a Thin Gold Film. Journal of Applied Physics, 86(11):6200, 1999. Cited on p. 60

[110] S. Debrus, J. Lafait, M. May, N. Pin con, D. Prot, C. Sella, and J. Venturini. Z-scan determination of the third-order optical nonlinearity of gold:silica nanocomposites. Journal of Applied Physics, 88(8):4469, 2000. Cited on p. 60 
[111] P. Wang, Y. Lu, L. Tang, J. Zhang, H. Ming, J. Xie, F.-H. Ho, H.-H. Chang, H.-Y. Lin, and D.-P. Tsai. Surface-enhanced optical nonlinearity of a gold film. Optics Communications, 229(1-6):425-429, January 2004. Cited on p. 60

[112] H. Shen, B. Cheng, G. Lu, T. Ning, D. Guan, Y. Zhou, and Z. Chen. Enhancement of optical nonlinearity in periodic gold nanoparticle arrays. Nanotechnology, 17(16):4274-7, August 2006. Cited on p. 60

[113] R. A. Ganeev, A. I. Ryasnyansky, S. R. Kamalov, M. K. Kodirov, and T. Usmanov. Nonlinear susceptibilities, absorption coefficients and refractive indices of colloidal metals. Journal of Physics D: Applied Physics, 34(11):1602-1611, June 2001. Cited on p. 60

[114] K. Nishikawa and M. Wakatani. PLASMA PHYSICS., volume 8. Springer, 2000. Cited on p. 60

[115] K. Bergmann, G. Schriever, O. Rosier, M. Müller, W. Neff, and R. Lebert. Highly repetitive, extreme-ultraviolet radiation source based on a gas-discharge plasma. Applied optics, 38(25):5413-7, September 1999. Cited on p. 60

[116] W. T. Silfvast. Comparison of radiation from laser-produced and dc-heated plasmas in xenon. Applied Physics Letters, 25(5):274, 1974. Cited on p. 60

[117] N. Damany, P. Laporte, J.-L. Subtil, and H. Damany. Multiphoton excitation and decay processes in xenon: Off-breakdown and breakdown emission at densities up to $1.4 \times 10^{21}$ atoms $\mathrm{cm}^{-3}$. Physical Review A, 32(6):3418-3424, December 1985. Cited on p. 60

[118] G. N. Gerasimov, B. E. Krylov, A. V. Loginov, and S. A. Shchukin. UV emission from excited inert-gas molecules. Soviet Physics Uspekhi, 35(5):400-419, May 1992. Cited on p. 60

[119] G. Cunge, B. Crowley, D. Vender, and M. M. Turner. Characterization of the E to $\mathrm{H}$ transition in a pulsed inductively coupled plasma discharge with internal coil geometry: bi-stability and hysteresis. Plasma Sources Science and Technology, 8(4):576-586, November 1999. Cited on p. 60, 61 
[120] I. M. El-Fayoumi, I. R. Jones, and M. M. Turner. Hysteresis in the E- to Hmode transition in a planar coil, inductively coupled $\mathrm{rf}$ argon discharge. Journal of Physics D: Applied Physics, 31(21):3082-3094, November 1998. Cited on p. 60

[121] K. Suzuki, K. Nakamura, H. Ohkubo, and H. Sugai. Power transfer efficiency and mode jump in an inductive RF discharge. Plasma Sources Science and Technology, 7(1):13-20, February 1998. Cited on p. 60

[122] S. Xu, K. N. Ostrikov, Y. Li, E. L. Tsakadze, and I. R. Jones. Low-frequency, highdensity, inductively coupled plasma sources: Operation and applications. Physics of Plasmas, 8(5):2549, 2001. Cited on p. 60

[123] T. Czerwiec and D. B. Graves. Mode transitions in low pressure rare gas cylindrical ICP discharge studied by optical emission spectroscopy. Journal of Physics D: Applied Physics, 37(20):2827-2840, October 2004. Cited on p. 60

[124] T. Popmintchev, M.-C. Chen, A. Bahabad, M. Gerrity, P. Sidorenko, O. Cohen, I. P. Christov, M. M. Murnane, and H. C. Kapteyn. Phase matching of high harmonic generation in the soft and hard X-ray regions of the spectrum. Proceedings of the National Academy of Sciences of the United States of America, 106(26):10516-21, June 2009. Cited on p. 68

[125] Georg-August University School of Science (GAUSS) Doctoral Degree Regulations (RerNat-O). http://www.gauss.uni-goettingen.de/RerNat-O_050712.pdf, June 2012. Cited on p. 83 


\section{Author contribution}

This chapter provides detailed information about the particular contributions of the author in the publications, which are reprinted in the Chapters 2-4. These three peer-reviewed articles fulfill the requirements to be considered in a cumulative thesis according to the doctoral degree regulations of the $\mathrm{PhD}$ program ProPhys in the Georg-August University School of Science (GAUSS) Göttingen [125]:

(1) M. Sivis, M. Duwe, B. Abel, and C. Ropers Nanostructure-enhanced atomic line emission, Nature 485, E1 (2012)

(2) M. Sivis, M. Duwe, B. Abel, and C. Ropers Extreme-ultraviolet light generation in plasmonic nanostructures, Nature Physics 9, 308 (2013)

(3) M. Sivis, and C. Ropers Generation and Bistability of a Waveguide Nanoplasma Observed by Enhanced Extreme-Ultraviolet Fluorescence, Physical Review Letters 111, 085001 (2013)

The publications listed above present original work by the author. In detail, this originality concerns the design, setup and conduction of all experiments presented in Publications (1), (2) and (3), as well as the analysis of the collected data. The sole exception displays the dark-field scattering measurements in Publication (2). The dark-field scattering setup was built by Matthias Duwe as a part of his diploma thesis, and the measurements presented in (2) were performed by Matthias Duwe in collaboration with the author. The fabrication of nanostructures, including the design and the gold film preparation, was done by the author, with helpful contributions from Matthias Duwe in the case of the bow-tie structures used in (1) and (2). The results were discussed and interpreted by all authors of the respective publications. The manuscripts of all publications were written by the author and Claus Ropers with contributions and revisions from Bernd Abel and Matthias Duwe on Articles (1) and (2). 



\section{Danksagung}

An erster Stelle gilt mein aufrichtiger Dank Prof. Dr. Claus Ropers für die hervorragende Betreuung während der Entstehung dieser Dissertation, seine Unterstützung in jeglicher Hinsicht und die zahlreichen erhellenden Diskussionen.

Mein Dank gilt gleichermaßen allen Mitgliedern der AG Ropers für die tolle Zusammenarbeit und gegenseitige Unterstützung. Insbesondere möchte ich Reiner Bormann, Matthias Duwe, Max Gulde, Georg Herink, Benjamin Schröder, Daniel Solli und Sergey Zayko an dieser Stelle namentlich erwähnen. In bester Erinnerung wird mir auch die gemeinsam verbrachte Zeit im Rahmen unserer vielen Gruppenaktivitäten bleiben.

Ferner danke ich allen Kolleginnen und Kollegen des IV. Physikalischen Instituts, des Instituts für Materialphysik und des SFB 755 für die gute Arbeitsatmosphäre und die technische Unterstützung. Allen Mitarbeitern der Werkstätten sei gedankt für ihre stets hervorragende Umsetzung meiner Aufträge.

Für die Korrekturen am Manuskript und zahlreiche wertvolle Ratschläge bin ich Georg Herink und Daniel Solli zutiefst verbunden.

Prof. Dr. Tim Salditt und Prof. Dr. Bernd Abel danke ich für die freundliche Übernahme des Koreferats dieser Arbeit.

Darüber hinaus bedanke ich mich bei Prof. Dr. Bernd Abel für die Kooperation zu Beginn dieser Doktorarbeit und bei Katrin Siefermann und Yaxing Liu für die gemeinsam durchgeführten Voruntersuchungen zu diesem Projekt.

Meinen Studienkollegen danke ich für eine unvergessliche Zeit während des gemeinsamen Studiums und vor allem für die vielen Stunden der Zerstreuung nebenher. Insbesondere gilt dies Marvin Walter, Benjamin Lenk, Georg Herink, Björn Hoffmann, Kai Ubben, Max-Julian Waack, Michael Mell und Steffen Ravekes.

Nicht zuletzt gilt meine tiefste Verbundenheit meiner Familie für das rege Interesse an meiner Arbeit. Im Besonderen danke ich meiner Frau Julia, und meinen beiden Töchtern Amira und Louisa, deren grenzenloses Verständnis und bedingungslose Unterstützung die intensive Arbeit an diesem Projekt überhaupt erst möglich gemacht haben. 